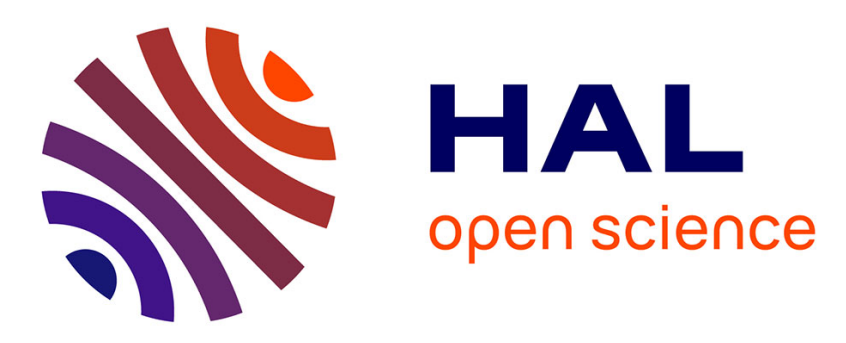

\title{
High order moment method for polydisperse evaporating sprays with mesh movement: application to internal combustion engines
}

Damien Kah, Oguz Emre, Quang Huy Tran, Stéphane de Chaisemartin, Stéphane Jay, Frédérique Laurent, Marc Massot

\section{To cite this version:}

Damien Kah, Oguz Emre, Quang Huy Tran, Stéphane de Chaisemartin, Stéphane Jay, et al.. High order moment method for polydisperse evaporating sprays with mesh movement: application to internal combustion engines. International Journal of Multiphase Flow, 2015, 71, pp.38-65. 10.1016/j.ijmultiphaseflow.2014.12.004 . hal-00941796v2

\section{HAL Id: hal-00941796 \\ https://hal.science/hal-00941796v2}

Submitted on 16 Jan 2015

HAL is a multi-disciplinary open access archive for the deposit and dissemination of scientific research documents, whether they are published or not. The documents may come from teaching and research institutions in France or abroad, or from public or private research centers.
L'archive ouverte pluridisciplinaire HAL, est destinée au dépôt et à la diffusion de documents scientifiques de niveau recherche, publiés ou non, émanant des établissements d'enseignement et de recherche français ou étrangers, des laboratoires publics ou privés. 


\title{
High order moment method for polydisperse evaporating sprays with mesh movement: application to internal combustion engines
}

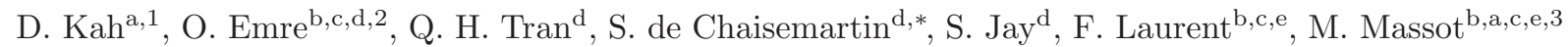 \\ ${ }^{a}$ Center for Turbulence Research, Stanford University, Stanford, CA 94305, USA \\ ${ }^{b}$ Laboratoire EM2C, CNRS, Grande Voie des Vignes, 92295 Châtenay-Malabry, France \\ ${ }^{c}$ École Centrale Paris, Grande Voie des Vignes, 92295 Châtenay-Malabry, France \\ ${ }^{d}$ IFP Energies nouvelles, 1 et 4 avenue de Bois-Préau, 92852 Rueil-Malmaison, France \\ ${ }^{e}$ Fédération de Mathématiques de l'Ecole Centrale Paris - FR CNRS 3487
}

\begin{abstract}
Relying on two recent contributions by Massot et al. [SIAM J. Appl. Math. 70 (2010), 3203-3234] and Kah et al. [J. Comput. Phys. 231 (2012), 394-422], where a Eulerian Multi-Size Moment (EMSM) model for the simulation of polydisperse evaporating sprays has been introduced, we investigate the potential of such an approach for the robust and accurate simulation of the injection of a liquid disperse phase into a gas for automotive engine applications. The original model used a high order moment method in droplet size to resolve polydispersity, with built-in realizability preserving numerical algorithm of high order in space and time, but only dealt with one-way coupling and was restricted to fixed meshes. Extending the approach to internal combustion engine and fuel injection requires solving two major steps forward, while preserving the properties of robustness, accuracy and realizability: 1- the extension of the method and numerical strategy to two-way coupling with stable integration of potential stiff source terms, 2- the introduction of a moving geometry and meshes. We therefore present a detailed account on how we have solved these two issues, provide a series of verification of the proposed algorithm, showing its potential in simplified configurations. The method is then implemented in the IFP-C3D unstructured solver for reactive compressible flows in engines and validated through comparisons with a structured fixed mesh solver. It finally proves its potential on a free spray jet injection where it is compared to a Lagrangian approach and its reliability and robustness are assessed, thus making it a good candidate for realistic injection applications.
\end{abstract}

Keywords: Eulerian models, polydispersity, high order moment method, realizability condition, ALE formalism, staggered moving mesh.

\section{Introduction}

Fully integrated reactive simulations with spray in internal combustion engines have become a critical target in the automotive industry, where CFD has an increasing impact in the decision process for the design of new prototypes. If a substantial level of maturity has been reached for the simulation of gases, it is not exaggerated to claim that the description of the fuel liquid jet in the chamber still requires major improvements.

\footnotetext{
${ }^{*}$ Corresponding author

Email addresses: damienk@stanford.edu (D. Kah), oguz.emre@ecp.fr (O. Emre), quang-huy.tran@ifpen.fr (Q. H. Tran), stephane.de-chaisemartin@ifpen.fr (S. de Chaisemartin), stephane.jay@ifpen.fr (S. Jay), frederique.laurent@ecp.fr (F. Laurent), marc.massot@ecp.fr (M. Massot)

${ }^{1}$ Supported by a PhD scholarship from IFP Energies nouvelles (2008-2011). Presently postdoctoral fellow at the Center for Turbulence Research, Stanford University.

${ }^{2}$ Supported by a PhD scholarship from IFP Energies nouvelles (2010-2013).

${ }^{3}$ The support of the France-Stanford Center for Interdisciplinary Studies through a collaborative project grant (P. Moin/M. Massot) is also gratefully acknowledged (2011-2012).
} 
There is, however, an imperious need to model the fuel liquid jet from the very moment it is injected until it eventually becomes a cloud of polydisperse droplets after secondary atomization. For instance, in reactive cases, the gaseous fuel mass fraction is a key parameter that monitors the final temperature and species concentration issued from the combustion process. Several key properties of the engines, such as its energetic and environmental efficiencies depend on these elements. Models relying on a DNS framework can capture the whole jet dynamics in the chamber for a limited range of Weber and Reynolds numbers. But both the computational resources required as well as the modeling and physics of topology changes at small scales, make it unaffordable in terms of the range of scales to be resolved for realistic conditions of industrial interest. Reduced order models are then a powerful alternative as it allows computations to be done at a reasonable cost, regardless of the chosen framework, such as LES or RANS.

Two families of reduced models can be found, based on different ground physical assumptions pertaining to the area under study. The first one, the class of two-fluid models, provides an Eulerian description of the dense fuel region close to the injector, referred to as separate phase flow $[3,57,36,1,40,42,44,13,12]$. It provides some level of description of the interface geometry depending on the modeling assumptions for potential equilibrium (see [37] for a general classification). The second modeling family relies on a statistical description of the particles/droplets far from injection when the atomization process has been fully completed. The particle properties are monitored by a number density function (NDF) that is solution of a kinetic equation $[26,47]$. Since the direct resolution of the kinetic equation is often intractable due to the large number of independent variables, stochastic Lagrangian methods "discretize" the NDF into "parcels", the dynamics of which is integrated using standard ODE solvers. This approach has been widely used and has shown to be efficient in numerous situations $[52,2,71,32,60,55]$. While quite accurate, its main drawback is the coupling of a Eulerian description for the gaseous phase to a Lagrangian description of the disperse phase, thus offering limited possibilities of vectorization/parallelization. Besides, as in any statistical approach, Lagrangian methods require a relatively large number of parcels to control statistical noise, especially in the unsteady and polydisperse configurations we target, and thus can be computationally expensive. One has to note that these difficulties are tackled within the community and recent improvements are to be found [8].

An alternative to the Lagrangian approach is an Eulerian moment method. Potentially, Eulerian methods can be a breakthrough in the aspects aforementioned, but the Eulerian description of some spray features seems less natural and calls for some endeavor. Polydispersity is one of these features. The closure of the velocity moments conditioned on size is classical and conducted through a hydrodynamic limit leading to an equilibrium velocity distribution, i.e., Maxwell-Boltzmann distribution up to zero temperature in the framework of direct numerical simulation $[41,9,38]$. Such an approach is known to be valid for Stokes number up to approximatively one, when droplet trajectory crossing is very limited [4]; beyond such a limit another closure has to be chosen (see [11, 63, 10, 46, 65, 62] and references therein) in order to describe statistical and deterministic trajectory crossing. Once a closure has been chosen in terms of velocity moments conditioned on droplet size, there are two options available for capturing the dynamics in the size phase space. One can either rely on size phase space discretization, with low order size moments in each section, as done in the multi-fluid approach developed in [9, 41, 63] from [31]. The multi-fluid model considers only one size moment which accounts for the liquid mass density on small intervals of the size phase space called sections. Formally, the disperse phase is composed of several fluids exchanging mass, momentum and heat with each other and with the gas through evaporation, drag and heat exchange. This model has shown to yield simple transport algorithms for transport in physical space in [9] implemented on parallel architectures [29]. Another path to the description of polydispersity is to increase the amount of information in each section such as in $[17,18]$ when a very accurate description of the size distribution is required. Potentially a hybrid method can be conceived such as in [39, 64], but the ultimate choice is to use a single section for evaporating sprays with a high order moment method in order to describe polydispersity, an alternative solution which represents a very interesting tool for automotive engine simulations.

At present, several moment methods have been designed in order to treat size polydispersity. The first one consists in solving the evolution of moments of a prescribed NDF, e.g., a log-normal law [51]. Presumably, this is very interesting since knowing the a priori profile of the NDF makes its reconstruction from the moments much easier. However, this assumption is restrictive in terms of the coverage of the physical processes. Moreover, this approach leads to serious numerical instabilities thus preventing its 
use for the treatment of an evaporating spray, since during the computation, a log-normal distribution function might not be reconstructed from the moment set dynamics. Another solution is high order moment methods, either quadrature method of moment (QMOM) where the dynamics of moments are evaluated after closing the source terms using quadrature methods (see [49] for example and references therein), or Direct Quadrature Method of Moment (DQMOM) [45] wherein equations are directly written on the quadrature weights and abscissas which describe the reconstructed distribution function having the same moments. Such methods have proved to be very efficient in a number of configurations, such as agglomeration, sintering, coagulation-fragmentation. But when it comes to accurately predicting the evaporating flux at zero size, which is a pointwise value to be reconstructed from the set of moments, these methods have shown their limits [27], even if some new developments have appeared [70], where some of the issues have been solved. The second problem is related to the transport in physical space. There exists a stumbling block for the usual approaches using high order moment method resolved with at least second-order finite volume methods. The transport algorithm does not systematically preserve the moment space, which means that slope reconstruction of moments by piecewise polynomials are likely to create vectors whose components fail to be the moments of a size distribution. One way to resolve this is to impose a posteriori to the moment vector to stay inside the realizable moment space, but this degrades the computational efficiency as well as the accuracy. We thus want to rely on robust and built-in realizable numerical methods and algorithms.

Further studies have been undertaken and both issues have been successfully addressed. First, for the treatment of the evaporation term, the high order moment method together with the associated numerical scheme presented in [48] proposes a solution with a high level of accuracy. It can be effectively used for quantitative predictions of an evaporating spray. Second, a high order transport scheme preserving the integrity of the moment set is provided in [39]. It makes use of two ingredients: the reconstruction of the independent canonical moments [16], as well as the use of an exact time integration scheme, thus guaranteeing that no truncation error is introduced. These modeling and algorithmic tools are referred to as the EMSM (Eulerian Multi-Size Moment) model. Its potential has been demonstrated through test cases using an academic solver. Such an approach has also been compared to the methods introduced in [66]. As explained in [39], both classes of aerosols and sprays can be described by this model ${ }^{4}$. However, these preliminary studies have been restricted to one-way coupling, where the preservation of the realizability condition and high order numerical methods are achieved through operator splitting and developed in the framework of fixed meshes, be it structured or unstructured. For the purpose of dealing with internal combustion engines and proving that these methods are good candidates for such configurations, these restrictions have to be alleviated and overcome.

In this paper, we focus on the spray class, even if most of what is investigated in the paper can be extended to aerosols. The purpose of the present contribution is three-fold. It aims firstly at extending the EMSM model to a two-way coupling framework, where the influence of the particles on the gas phase is taken into account, while maintaining the properties of the numerical strategy adopted so far, a robust and accurate resolution with built-in realizability preserving algorithms, as well as the ability of coping with very stiff source terms through implicit resolution. The second objective is to extend the numerical methods to moving computational domains required in the field of internal combustion engines (ICE). The latter objective is naturally motivated by the observation that the combustion chamber, where fuel injection takes place, is bounded by a moving piston. The appropriate tool to help us take up this challenge is the Arbitrary Lagrangian-Eulerian (ALE) formalism [19, 34, 35, 24], which lies at the heart of the industrial software IFP-C3D [5] dedicated to compressible and reactive flows under mesh motion. IFP-C3D relies on a RANS formalism for the gas for industrial configurations but the numerical schemes implemented can be also potentially used for unsteady laminar DNS calculations. Considering the amount of material needed in order to reach the proposed objectives, we have decided to restrict ourselves to simplified models (Stokes drag, no heat exchange except through evaporation, simple evaporation laws) in order to prove the efficiency and potential of the proposed method, while the extension to complex

\footnotetext{
${ }^{4}$ What distinguishes these classes is their Stokes number based on some typical gas flow time. It is very small for aerosol, thus particles are transported at the carrier phase velocity. But for spray particles the Stokes number reaches higher values, accounting for the fact that they have their own dynamics.
} 
models used in realistic configurations, as well as the detailed analysis of the convergence rate of the proposed methods, are out of the scope of this paper. The third objective is to implement the method in the industrial code IFP-C3D and to adapt the new numerical methods to comply with the code structure, in order to propose a series of simulations in realistic injection configurations, where we can assess the achievements of the method in comparison with Lagrangian simulations classically available in the code. This should prove the potential of Eulerian high order moment models and related resolution strategy for ICE simulations.

The paper is then organized as follows. We first derive the two-way coupled EMSM model from the kinetic level of description. Section 3 is devoted to an overview of the numerical method, where we highlight the new numerical strategy in order to cope with stiff source terms implicitly while preserving the robustness of the original method. Then the splitting strategy, in the ALE formalism, is introduced. Specific details at the fully discrete level are provided in an Appendix in order to allow the possibility of reproducing the proposed simulations conducted in the paper. The theoretical and numerical issues associated with each phase are discussed, with a particular emphasis laid on the realizability of moments. In $\S 4$, we focus on the two novel features of the proposed method, namely the two-way coupling and the transport of moments, and provide a set of verifications configurations. For each feature, we present numerical results in simplified situations to illustrate the capability of the numerical algorithms. The implementation of the method in the unstructured, industrial software IFP-C3D as well as the related validation simulations by comparison with the 3-D structured mesh academic code MUSES3D [28] are conducted in $\S 5$, in a laminar one-way coupled DNS simulation. The stability through mesh movement is assessed, evaporation and transport implemented algorithms are validated by a comparison with a DNS computation using a completely different numerical algorithm. Finally, the feasibility of a spray injection simulation using IFP-C3D with a RANS model for the gaseous phase, and two-way coupling on the non-fluctuating quantities, is demonstrated in $\S 6$, paving the way for computations in real injection conditions, once a consistent two-way coupled turbulence model will be available [23].

\section{Derivation of the EMSM model}

The purpose of the present section is to derive the macroscopic Eulerian model for the spray. As done in [39], the derivation starts from a kinetic equation describing the spray at the mesoscopic scale. A closure assumption in velocity phase space conditioned in droplet size is first introduced, leading to an intermediate semi-kinetic system of conservation equations. A high order moment method in size is used to come up with the final macroscopic conservation laws. In the present paper, we will restrict ourselves to simple models, for the sake of legibility. The proposed approach can be extended easily to more complex models. The underlying assumptions and physical validity of the proposed approach are highlighted in this section. We introduce a non-dimensional form of the equations and show some key properties satisfied at the continuous level by the obtained systems of conservation equations. We will take benefit of these properties to build a dedicated numerical method described in part 3 .

\subsection{From the kinetic equation to a semi-kinetic system}

We take the general point of view of a dilute droplet spray described by a number density function (NDF) $f$ such that

$$
f(t, \boldsymbol{x}, S, \boldsymbol{u}, T) \mathrm{d} \boldsymbol{x} \mathrm{d} S \mathrm{~d} \boldsymbol{u} \mathrm{d} T,
$$

represents, for each time $t$, the number of particles lying at position $\boldsymbol{x}=\left(x_{1}, x_{2}, x_{3}\right)$ within a volume element $\mathrm{d} \boldsymbol{x}$, moving at velocity $\boldsymbol{u}=\left(v_{1}, v_{2}, v_{3}\right)$ within a velocity element $\mathrm{d} \boldsymbol{u}$, having size $S$ and temperature $T$ within the corresponding phase element $\mathrm{d} S \mathrm{~d} T$. In the applications under consideration, particles undergo evaporation and drag. In such a context, the NDF $f$ is governed by the kinetic equation

$$
\partial_{t} f+\nabla_{\boldsymbol{x}} \cdot(\boldsymbol{u} f)-\partial_{S}(K f)+\partial_{T}(Q f)+\nabla_{\boldsymbol{v}} \cdot(\boldsymbol{F} f)=0
$$

which is also referred to as the Williams-Boltzmann equation [67]. In the left side of equation (2.1), the second term expresses the transport in the physical space, the third term reflects the droplet evaporation, the fourth term accounts for the heat exchange with the surrounding gas, and the fifth term stands for the drag exerted by the gas. The scalars $K$ and $Q$ are, respectively, the evaporation rate (at which the 
droplet surface decreases) and the droplet energy change rate. As for the vector $\boldsymbol{F}$, it is the droplet acceleration. It is taken for granted that $K, Q$ and $\boldsymbol{F}$ are given functions of $(t, \boldsymbol{x}, S, \boldsymbol{u}, T)$. In fact, since $\boldsymbol{F}$ is the drag force due to the gas, it also depends on some extra variables associated with the gas phase.

What we do exactly mean by the "size" $S$ of each droplet particle is its surface. As the particles are assumed to be spherical, we could also have decided to work with their radius $r$ or their volume $V$. With a slight abuse of notation, we would of course have had

$$
f(t, \boldsymbol{x}, r, \boldsymbol{u}, T) \mathrm{d} r=f(t, \boldsymbol{x}, S, \boldsymbol{u}, T) \mathrm{d} S=f(t, \boldsymbol{x}, V, \boldsymbol{u}, T) \mathrm{d} V,
$$

upon the change of variables

$$
V=\frac{4}{3} \pi r^{3} \quad \text { and } \quad S=4 \pi r^{2} .
$$

As mentioned in the literature describing kinetic-based spray model derivations ([37] for instance), the use of spray models based on a Williams-Boltzmann kinetic equation is associated with various assumptions:

- droplets are considered as point particles, thus no finite size effect is taken into account and no volume fraction appears in gas phase equations;

- long distance interactions are not accounted for.

In the present contribution, the computation configurations involve a relatively low spray volume fraction $\left(2.5 \cdot 10^{-4}\right)$ and mass loading (around 0.2$)$. In these conditions, the previous assumptions stand. These conditions will also justify the way we consider 2-way coupling, through source terms due to the spray in the gas equations, see for example [41].

To close the model derived in the following, we choose to limit ourselves to simple spray physical models: say d-square law evaporation model and Stokes drag force. As previously said this restriction is not a limit of the approach, but it allows to focus on the difficulties of the derivation itself. No additional difficulties are to be found when coupling with more realistic models.

Starting from the microscopic model (2.1), our purpose is to work out a closed system of equations involving the total number density

$$
n(t, \boldsymbol{x}, S)=\iint_{\mathbb{R}^{3} \times \mathbb{R}^{+}} f(t, \boldsymbol{x}, S, \boldsymbol{u}, T) \mathrm{d} \boldsymbol{u} \mathrm{d} T,
$$

the mean particle velocity

$$
\boldsymbol{u}_{\boldsymbol{d}}(t, \boldsymbol{x}, S)=\frac{1}{n(t, \boldsymbol{x}, S)} \iint_{\mathbb{R}^{3} \times \mathbb{R}^{+}} f(t, \boldsymbol{x}, S, \boldsymbol{u}, T) \boldsymbol{u} \mathrm{d} \boldsymbol{u} \mathrm{d} T,
$$

and the mean particle temperature

$$
T_{d}(t, \boldsymbol{x}, S)=h_{d}^{-1}\left(\frac{1}{n(t, \boldsymbol{x}, S)} \iint_{\mathbb{R}^{3} \times \mathbb{R}^{+}} f(t, \boldsymbol{x}, S, \boldsymbol{u}, T) h_{d}(T) \mathrm{d} \boldsymbol{u} \mathrm{d} T\right)
$$

for some enthalpy density $h_{d}$, required to be increasing (therefore invertible) function of the temperature. This system is said to be semi-kinetic. To this end, and in view of the closure assumption made in the Multi-Fluid model [41], we assume $f$ to be of the form

$$
f(t, \boldsymbol{x}, S, \boldsymbol{u}, T)=n(t, \boldsymbol{x}, S) \delta\left(T-T_{d}(t, \boldsymbol{x}, S)\right) \delta\left(\boldsymbol{u}-\boldsymbol{u}_{\boldsymbol{d}}(t, \boldsymbol{x}, S)\right) .
$$

In other words, $f$ is a distribution with a single velocity and a single temperature conditioned by the size. This corresponds to a regime when the particle Stokes number - that is particle inertia with respect to a typical gaseous flow time scale, which will be defined in $\S 2.3$ - is small enough in order to neglect the velocity dispersion among particles, yet large enough so that the particles have their own velocity, 
different from the gas one $\mathrm{e}^{5}$. According to the classification established by Balachandar and Eaton in [4], this assumption is applicable for a range of Stokes between 0.2 and 1 . For more inertial particles, Lagrangian methods can be thought of, as well as more refined Eulerian approaches, see for example $[62,65,10,46]$.

The semi-kinetic equations are obtained by integrating (2.1) with respect to $(\boldsymbol{u}, T)$ after multiplying it by $1, \boldsymbol{u}$, and $h_{d}(T)$. Thanks to the mono-kinetic assumption (2.4), this operation yields

$$
\begin{array}{lr}
\partial_{t}(n)+\nabla_{\boldsymbol{x}} \cdot\left(n \boldsymbol{u}_{\boldsymbol{d}}\right) & -\partial_{S}(K n) \\
\partial_{t}\left(n \boldsymbol{u}_{\boldsymbol{d}}\right)+\nabla_{\boldsymbol{x}} \cdot\left(n \boldsymbol{u}_{\boldsymbol{d}} \otimes \boldsymbol{u}_{\boldsymbol{d}}\right)-\partial_{S}\left(K n \boldsymbol{u}_{\boldsymbol{d}}\right)-n \boldsymbol{F}_{\boldsymbol{d}} & =0 \\
\partial_{t}\left(n h_{d}\right)+\nabla_{\boldsymbol{x}} \cdot\left(n h_{d} \boldsymbol{u}_{\boldsymbol{d}}\right) & -\partial_{S}\left(K n h_{d}\right)-n C_{p, l} Q=0
\end{array}
$$

where $H, K, \boldsymbol{F}_{\boldsymbol{d}}$ are now evaluated at $\left(t, \boldsymbol{x}, S, \boldsymbol{u}_{\boldsymbol{d}}, T_{d}\right)$, and $C_{p, l}\left(T_{d}\right)=h_{d}^{\prime}\left(T_{d}\right)$ is the average heat capacity of the liquid. The actual value of $\boldsymbol{F}_{\boldsymbol{d}}$, given by the Stokes law

$$
\boldsymbol{F}_{\boldsymbol{d}}\left(S, \boldsymbol{u}_{\boldsymbol{d}}\right)=\frac{18 \pi \mu_{g}}{\rho S}\left(\boldsymbol{u}_{\boldsymbol{g}}-\boldsymbol{u}_{\boldsymbol{d}}\right)
$$

involves two quantities coming from the surrounding gas, namely, its dynamic viscosity $\mu_{g}$ and its local velocity $\boldsymbol{u}_{\boldsymbol{g}}$. We are thus led to momentarily leave aside the spray system and to elaborate on the equations for the gas phase.

The gas is modeled by the compressible Navier-Stokes system, augmented with sources terms describing the interaction with the particles, i.e.,

$$
\begin{aligned}
& \partial_{t}\left(\rho_{g}\right)+\nabla_{\boldsymbol{x}} \cdot\left(\rho_{g} \boldsymbol{u}_{\boldsymbol{g}}\right) \\
& \mathfrak{S}^{\rho}, \\
& \partial_{t}\left(\rho_{g} \boldsymbol{u}_{\boldsymbol{g}}\right)+\nabla_{\boldsymbol{x}} \cdot\left(\rho_{g} \boldsymbol{u}_{\boldsymbol{g}} \otimes \boldsymbol{u}_{\boldsymbol{g}}+P_{g} \boldsymbol{I}\right)=\mathfrak{S}^{\rho \boldsymbol{u}}+\nabla_{\boldsymbol{x}} \cdot\left(\boldsymbol{\Sigma}_{\boldsymbol{g}}\right), \\
& \partial_{t}\left(\rho_{g} E_{g}\right)+\nabla_{\boldsymbol{x}} \cdot\left(\rho_{g} E_{g} \boldsymbol{u}_{\boldsymbol{g}}+P_{g} \boldsymbol{u}_{\boldsymbol{g}}\right)=\mathfrak{S}^{\rho e}+\nabla_{\boldsymbol{x}} \cdot\left(\boldsymbol{\Sigma}_{\boldsymbol{g}} \cdot \boldsymbol{u}_{\boldsymbol{g}}\right),
\end{aligned}
$$

where $\boldsymbol{I}$ is the identity tensor, $\boldsymbol{\Sigma}_{\boldsymbol{g}}=\mu_{g}\left(T_{g}\right)\left(\nabla_{\boldsymbol{x}} \boldsymbol{u}_{\boldsymbol{g}}+\nabla_{\boldsymbol{x}} \boldsymbol{u}_{\boldsymbol{g}}{ }^{T}\right)-\frac{2}{3} \mu_{g}\left(T_{g}\right)\left(\nabla_{\boldsymbol{x}} \cdot \boldsymbol{u}_{\boldsymbol{g}}\right) \boldsymbol{I}$ is the classical Newtonian viscous stress tensor, and (see [37, p. 41])

$$
\begin{aligned}
\mathfrak{S}^{\rho} & =\int \rho_{d} V^{\prime} K n \mathrm{~d} S, \\
\mathfrak{S}^{\rho \boldsymbol{u}} & =\int \rho_{d} V^{\prime} K n \boldsymbol{u}_{\boldsymbol{d}} \mathrm{d} S-\int \rho_{d} V n \boldsymbol{F}_{\boldsymbol{d}} \mathrm{d} S, \\
\mathfrak{S}^{\rho e} & =\int \rho_{d} V^{\prime} K n h_{d} \mathrm{~d} S-\int \rho_{d} V n C_{p, l} H \mathrm{~d} S .
\end{aligned}
$$

In formulae (2.8), as well as in equation (2.6), the symbol $\rho$ denotes the density of the liquid droplets. This density is assumed to be a given data. We recall that $V$ is the volume of a droplet. Its value was given in equation (2.2) as a function of the radius $r$, but here must be thought of as a function of the surface $S$. More explicitly, we have

$$
V(S)=\frac{S^{3 / 2}}{6 \pi^{1 / 2}}, \quad \text { with } \quad V^{\prime}(S)=\frac{S^{1 / 2}}{4 \pi^{1 / 2}} .
$$

For simplicity, we shall restrict ourselves to an ideal gas governed by the law of state

$$
P_{g}=\left(\gamma_{g}-1\right) \rho_{g}\left(E_{g}-\frac{1}{2}\left\|\boldsymbol{u}_{\boldsymbol{g}}\right\|^{2}\right) \quad \text { and } \quad T_{g}=\frac{P_{g}}{R_{g} \rho_{g}},
$$

for some $\gamma_{g}>1$ and $R_{g}>0$. Note that, while the gas unknown functions $\rho_{g}$ (density), $\boldsymbol{u}_{\boldsymbol{g}}$ (velocity), $E_{g}$ (total energy) depend merely on $(t, \boldsymbol{x})$, the spray unknown functions $n, \boldsymbol{u}_{\boldsymbol{d}}, h_{d}$ depend on $(t, \boldsymbol{x}, S)$.

\footnotetext{
${ }^{5}$ Let us underline that if this property is satisfied at time $t=0$ and under some reasonable assumption on the gaseous velocity phase, which were mathematically characterized in [9, 47], this property is preserved as long as the Stokes number of the particles is below a critical Stokes number and there is a one-to-one correspondence between the kinetic or mesoscopic level of description and the macroscopic one, as for the normal solutions of the Botlzmann equation in the hydrodynamic limit.
} 


\subsection{From the semi-kinetic system to the multi-size moment system}

The semi-kinetic system (2.5) for droplets is already a significant step forward but still has a continuous size phase space of dimension one. Our ultimate goal, however, is to simplify it further into a macroscopic model under the form of a system of conservation equations, the unknowns of which are moment in size, that is functions of only $(t, \boldsymbol{x})$. In the transformation process, it is naturally desirable that the new system could somehow "remember" the effect of the size $S$. For the sake of legibility of the paper, we will introduce a set of stronger hypotheses. In addition to the ansatz (2.4), we now prescribe that

$$
\boldsymbol{u}_{\boldsymbol{d}}(t, \boldsymbol{x}, S)=\boldsymbol{u}_{\boldsymbol{d}}(t, \boldsymbol{x}) \quad \text { and } \quad K\left(t, \boldsymbol{x}, S, \boldsymbol{u}_{\boldsymbol{d}}, T_{d}\right)=K\left(t, \boldsymbol{x}, \boldsymbol{u}_{\boldsymbol{d}}, T_{d}\right) .
$$

To put it another way, neither the particle velocity nor the evaporation rate depend on the size variable. More advanced modeling is available with Eulerian moment method approaches, and size-velocity correlation could have been taken into account enriching the present model as done in [63]. We choose not to introduce this further modeling, requiring more complex algebraic computations, and to present the concepts on simplified test cases. Such a extension will be thought of later on.

This enables us to easily derive conservation laws for the moments, defined as

$$
m_{k}(t, \boldsymbol{x})=\int S^{k} n(t, \boldsymbol{x}, S) \mathrm{d} S, \quad k \in \mathbb{N} .
$$

A straightforward calculation shows that

$$
\partial_{t}\left(m_{0}\right)+\nabla_{\boldsymbol{x}} \cdot\left(m_{0} \boldsymbol{u}_{\boldsymbol{d}}\right)=-K n(t, \boldsymbol{x}, S=0),
$$

for $k=0$, with $K n(t, \boldsymbol{x}, S=0)$ being the disappearance rate of droplets through evaporation, and

$$
\partial_{t}\left(m_{k}\right)+\nabla_{\boldsymbol{x}} \cdot\left(m_{k} \boldsymbol{u}_{\boldsymbol{d}}\right)=-k K m_{k-1}
$$

for $k \geq 1$.

In practice, we will work with a sequence of $N+1$ size moments, $\boldsymbol{m}=\left(m_{0}, m_{1}, \ldots, m_{N}\right)$, the number of moments used being chosen for a good compromise between precision and computational cost related to involved algebraic computations. We need to derive a self-consistent system of conservation equations with source terms, which does not involve any more the relation to the kinetic level of description. This gives rise to the problem of designing a suitable approximation of $n$ from the knowledge of the moment sequence, the value at $S=0$ appearing in the right-hand side of equation (2.13a). A solution to this problem was proposed by Massot et al. [48]. By performing an entropy maximization [50] in the sense of information theory, it is possible to reconstruct a unique distribution function $\tilde{n}(\boldsymbol{m}, S)$, the $N+1$ first moments of which coincide with $\left(m_{0}, m_{1}, \ldots, m_{N}\right)[50,64]$. At this level, we should rewrite a whole set of equations on the related approximation of the size moments we are working with. However, with a slight abuse of notation for the sake of legibility, we will keep the original notations and replace equation (2.13a) by its approximated counterpart, which is autonomous from the original kinetic level of description :

$$
\partial_{t}\left(m_{0}\right)+\nabla_{\boldsymbol{x}} \cdot\left(m_{0} \boldsymbol{u}_{\boldsymbol{d}}\right)=-K \tilde{n}(\boldsymbol{m}, 0) .
$$

Another straightforward calculation shows that

$$
\partial_{t}\left(m_{0} \boldsymbol{u}_{\boldsymbol{d}}\right)+\nabla_{\boldsymbol{x}} \cdot\left(m_{0} \boldsymbol{u}_{\boldsymbol{d}} \otimes \boldsymbol{u}_{\boldsymbol{d}}\right)=\int \boldsymbol{F}_{\boldsymbol{d}} n \mathrm{~d} S,
$$

for $k=0$, and

$$
\partial_{t}\left(m_{k} \boldsymbol{u}_{\boldsymbol{d}}\right)+\nabla_{\boldsymbol{x}} \cdot\left(m_{k} \boldsymbol{u}_{\boldsymbol{d}} \otimes \boldsymbol{u}_{\boldsymbol{d}}\right)=\int S^{k} \boldsymbol{F}_{\boldsymbol{d}} n \mathrm{~d} S-k K m_{k-1} \boldsymbol{u}_{\boldsymbol{d}},
$$

for $k \geq 1$. Of these equations, we keep only that corresponding to $k=1$.

The rationale for such a choice comes from several reasons. First we work with integer surface moments for both physical reasons related to evaporation, drag and heat transfer, coupled to computational efficiency and accuracy reasons (see [48, 39]). Besides, we want to involve only conservative variables for the accurate and stable treatment of the convective terms. Finally, out of the conserved size moments, 
the form (2.6) of the drag $\boldsymbol{F}_{\boldsymbol{d}}$ acceleration is in favor of $k=1$. Thus the natural form of the momentum evolution equation reads

$$
\partial_{t}\left(m_{1} \boldsymbol{u}_{\boldsymbol{d}}\right)+\nabla_{\boldsymbol{x}} \cdot\left(m_{1} \boldsymbol{u}_{\boldsymbol{d}} \otimes \boldsymbol{u}_{\boldsymbol{d}}\right)=\frac{18 \pi}{\rho} \mu_{g} m_{0}\left(\boldsymbol{u}_{\boldsymbol{g}}-\boldsymbol{u}_{\boldsymbol{d}}\right)-k K m_{0} \boldsymbol{u}_{\boldsymbol{d}}
$$

Nonetheless, in order to ensure total mass and momentum conservation, half-integral moments will be involved. Indeed the mass of the spray is obtained by the $3 / 2$-order size moment ; spray mass and momentum conservations read

$$
\begin{aligned}
& \partial_{t}\left(m_{3 / 2}\right)+\nabla_{\boldsymbol{x}} \cdot\left(m_{3 / 2} \boldsymbol{u}_{\boldsymbol{d}}\right) \quad=-\frac{3}{2} K m_{1 / 2}, \\
& \partial_{t}\left(m_{3 / 2} \boldsymbol{u}_{\boldsymbol{d}}\right)+\nabla_{\boldsymbol{x}} \cdot\left(m_{3 / 2} \boldsymbol{u}_{\boldsymbol{d}} \otimes \boldsymbol{u}_{\boldsymbol{d}}\right)=-\frac{3}{2} K m_{1 / 2} \boldsymbol{u}_{\boldsymbol{d}}+\left(18 \pi / \rho_{d}\right) \mu_{g} m_{1 / 2}\left(\boldsymbol{u}_{\boldsymbol{g}}-\boldsymbol{u}_{\boldsymbol{d}}\right) .
\end{aligned}
$$

Combining (2.17) with equations (2.7a)-(2.7b) of the gas, which spell out as

$$
\begin{aligned}
\partial_{t}\left(\rho_{g}\right)+\nabla_{\boldsymbol{x}} \cdot\left(\rho_{g} \boldsymbol{u}_{\boldsymbol{g}}\right) & =\frac{1}{4 \pi^{1 / 2}} \rho_{d} K m_{1 / 2}, \\
\partial_{t}\left(\rho_{g} \boldsymbol{u}_{\boldsymbol{g}}\right)+\nabla_{\boldsymbol{x}} \cdot\left(\rho_{g} \boldsymbol{u}_{\boldsymbol{g}} \otimes \boldsymbol{u}_{\boldsymbol{g}}+P_{g} \boldsymbol{I}\right) & =\frac{1}{4 \pi^{1 / 2}} \rho_{d} K m_{1 / 2} \boldsymbol{u}_{\boldsymbol{d}}-\frac{18 \pi}{6 \pi^{1 / 2}} \mu_{g} m_{1 / 2}\left(\boldsymbol{u}_{\boldsymbol{g}}-\boldsymbol{u}_{\boldsymbol{d}}\right)+\nabla_{\boldsymbol{x}} \cdot\left(\boldsymbol{\Sigma}_{\boldsymbol{g}}\right),
\end{aligned}
$$

the zeroth-order source terms cancel out and we end up with the conservation laws

$$
\begin{array}{ll}
\partial_{t}\left(\rho_{g}+\frac{1}{6 \pi^{1 / 2}} \rho_{d} m_{3 / 2}\right)+\nabla_{\boldsymbol{x}} \cdot\left(\rho_{g} \boldsymbol{u}_{\boldsymbol{g}}+\frac{1}{6 \pi^{1 / 2}} \rho_{d} m_{3 / 2} \boldsymbol{u}_{\boldsymbol{d}}\right) & =0, \\
\partial_{t}\left(\rho_{g} \boldsymbol{u}_{\boldsymbol{g}}+\frac{1}{6 \pi^{1 / 2}} \rho_{d} m_{3 / 2} \boldsymbol{u}_{\boldsymbol{d}}\right)+\nabla_{\boldsymbol{x}} \cdot\left(\rho_{g} \boldsymbol{u}_{\boldsymbol{g}} \otimes \boldsymbol{u}_{\boldsymbol{g}}+P_{g} \boldsymbol{I}+\frac{1}{6 \pi^{1 / 2}} \rho_{d} m_{3 / 2} \boldsymbol{u}_{\boldsymbol{d}} \otimes \boldsymbol{u}_{\boldsymbol{d}}\right)=\nabla_{\boldsymbol{x}} \cdot\left(\boldsymbol{\Sigma}_{\boldsymbol{g}}\right),
\end{array}
$$

for the total mass and total momentum. Let us underline that these half-integral moments are functions of the vector of conserved integer moments $\boldsymbol{m}$ through $\tilde{n}(\boldsymbol{m}, t)$ and will thus be used in the method and system (2.19) and will be satisfied in the numerical method.

Returning to the multi-moment model, we now make a new assumption by asserting that

$$
T_{d}(t, \boldsymbol{x}, S)=T_{d 0} \quad \text { and } \quad H\left(t, \boldsymbol{x}, S, \boldsymbol{u}_{\boldsymbol{d}}, T_{d}\right)=0 .
$$

The spray flow is assumed isothermal and the heat transfer term can be neglected [59, 30]. Contrary to (2.4) and (2.11), the last assumption (2.20) is in no way essential. It is meant to help us obtaining a simplest possible macroscopic model. More complex models can be envisaged [48]. As a matter of fact, even assumption (2.11) can be relaxed and models with size-dependent velocity can be considered, as exemplified in the recent work of Vié et al. [64]. But let us go back to assumption (2.20) and see what it implies. From $T_{d}=T_{d 0}$, we infer that $h_{d}=h_{d}\left(T_{d 0}\right)$ is a also constant. Combining this with $H=0$, it clearly appears that the enthalpy equation $(2.5 \mathrm{c})$ is no more than a scalar multiple of the number density equation (2.5a). Being redundant, the enthalpy equation can be left out.

Let us recapitulate the EMSM model. It consists of two coupled sets of PDEs, the unknowns of which are functions of $(t, \boldsymbol{x})$.

- For the spray, find $\left(\boldsymbol{m}, \boldsymbol{u}_{\boldsymbol{d}}\right)=\left(m_{0}, m_{1}, \ldots, m_{N}, \boldsymbol{u}_{\boldsymbol{d}}\right)$ such that

$$
\begin{array}{lll}
\partial_{t}\left(m_{0}\right) & +\nabla_{\boldsymbol{x}} \cdot\left(m_{0} \boldsymbol{u}_{\boldsymbol{d}}\right) & =-K \tilde{n}(\boldsymbol{m}, S=0), \\
\partial_{t}\left(m_{1}\right) & +\nabla_{\boldsymbol{x}} \cdot\left(m_{1} \boldsymbol{u}_{\boldsymbol{d}}\right) & =-K m_{0}, \\
\partial_{t}\left(m_{2}\right) & +\nabla_{\boldsymbol{x}} \cdot\left(m_{2} \boldsymbol{u}_{\boldsymbol{d}}\right) & =-2 K m_{1}, \\
\vdots & \vdots & \vdots \\
\partial_{t}\left(m_{N}\right) & +\nabla_{\boldsymbol{x}} \cdot\left(m_{N} \boldsymbol{u}_{\boldsymbol{d}}\right) & =-N K m_{N-1}, \\
\partial_{t}\left(m_{1} \boldsymbol{u}_{\boldsymbol{d}}\right)+\nabla_{\boldsymbol{x}} \cdot\left(m_{1} \boldsymbol{u}_{\boldsymbol{d}} \otimes \boldsymbol{u}_{\boldsymbol{d}}\right)=-K m_{0} \boldsymbol{u}_{\boldsymbol{d}}+\frac{18 \pi}{\rho} \mu_{g} m_{0}\left(\boldsymbol{u}_{\boldsymbol{g}}-\boldsymbol{u}_{\boldsymbol{d}}\right),
\end{array}
$$

where $\tilde{n}(\boldsymbol{m}, S)$ is the entropy-maximizing reconstructed distribution [48]. In this paper, we set $N=3$. This choice was shown [39] to be a good compromise between accuracy and efficiency. 
- For the gas, find $\left(\rho_{g}, \boldsymbol{u}_{\boldsymbol{g}}, E_{g}\right)$ such that

$$
\begin{aligned}
& \partial_{t}\left(\rho_{g}\right)+\nabla_{\boldsymbol{x}} \cdot\left(\rho_{g} \boldsymbol{u}_{\boldsymbol{g}}\right) \quad=\tilde{\mathfrak{S}}^{\rho}, \\
& \partial_{t}\left(\rho_{g} \boldsymbol{u}_{\boldsymbol{g}}\right)+\nabla_{\boldsymbol{x}} \cdot\left(\rho_{g} \boldsymbol{u}_{\boldsymbol{g}} \otimes \boldsymbol{u}_{\boldsymbol{g}}+P_{\boldsymbol{g}} \boldsymbol{I}\right)=\tilde{\mathfrak{S}}^{\rho \boldsymbol{u}}+\nabla_{\boldsymbol{x}} \cdot\left(\boldsymbol{\Sigma}_{\boldsymbol{g}}\right), \\
& \partial_{t}\left(\rho_{g} E_{g}\right)+\nabla_{\boldsymbol{x}} \cdot\left(\rho_{g} E_{g} \boldsymbol{u}_{\boldsymbol{g}}+P_{g} \boldsymbol{u}_{\boldsymbol{g}}\right)=\tilde{\mathfrak{S}}^{\rho e}+\nabla_{\boldsymbol{x}} \cdot\left(\boldsymbol{\Sigma}_{\boldsymbol{g}} \cdot \boldsymbol{u}_{\boldsymbol{g}}\right),
\end{aligned}
$$

where

$$
\begin{aligned}
\tilde{\mathfrak{S}}^{\rho} & =\int \rho_{d} V^{\prime} K \tilde{n} \mathrm{~d} S & =\frac{\rho_{d}}{4 \pi^{1 / 2}} K \tilde{m}_{1 / 2}, \\
\tilde{\mathfrak{S}}^{\rho \boldsymbol{u}} & =\int \rho_{d} V^{\prime} K \tilde{n} \boldsymbol{u}_{\boldsymbol{d}} \mathrm{d} S-\int \rho_{d} V \tilde{n} \boldsymbol{F}_{\boldsymbol{d}} \mathrm{d} S & =\frac{\rho_{d}}{4 \pi^{1 / 2}} K \tilde{m}_{1 / 2} \boldsymbol{u}_{\boldsymbol{d}}-\frac{18 \pi}{6 \pi^{1 / 2}} \mu_{g} \tilde{m}_{1 / 2}\left(\boldsymbol{u}_{\boldsymbol{g}}-\boldsymbol{u}_{\boldsymbol{d}}\right), \\
\tilde{\mathfrak{S}}^{\rho e} & =\int \rho_{d} V^{\prime} K \tilde{n} h_{d} \mathrm{~d} S & =\frac{\rho_{d}}{4 \pi^{1 / 2}} K \tilde{m}_{1 / 2} h_{d},
\end{aligned}
$$

are the reconstructed counterparts of the original source terms (2.8) under the constraint $H=0$. The ideal law of state (2.10) holds and the viscous stress tensor $\boldsymbol{\Sigma}_{\boldsymbol{g}}$ is defined as before.

The full model (2.21)-(2.23) is called two-way coupling model. A simplified version, called oneway coupling model, is obtained by setting $\tilde{\mathfrak{S}}^{\rho}=\tilde{\mathfrak{S}}^{\rho u}=\tilde{\mathfrak{S}}^{\rho e}=0$. The one-way coupling model was investigated by Kah et al. [39] in order to demonstrate the potential of the EMSM approach regarding the dynamics of size moments.

\subsection{Nondimensional formulation}

To gain some insight, we formulate the above system with non-dimensional variables. Let $L_{0}$ be a reference length, $U_{0}$ be a reference velocity, and $S_{0}$ be a reference droplet size. We remark that $S_{0}$, usually taken to be the maximum size of droplets, can be very different from $L_{0}^{2}$ (or $4 \pi L_{0}^{2}$ ), taken for example as an injector diameter in the applications considered in the paper. We introduce the dimensionless quantities

$$
\begin{array}{rlrlrl}
t^{*} & =\frac{U_{0}}{L_{0}} t, & \boldsymbol{x}^{*}=\frac{1}{L_{0}} \boldsymbol{x}, & \boldsymbol{u}_{\boldsymbol{d}}^{*}=\frac{1}{U_{0}} \boldsymbol{u}_{\boldsymbol{d}}, & S^{*}=\frac{1}{S_{0}} S, & h^{*}=\frac{1}{U_{0}^{2}} h, \\
n^{*}=L_{0}^{3} S_{0} n, & \tilde{n}^{*}=L_{0}^{3} S_{0} \tilde{n}, & m_{k}^{*}=\frac{L_{0}^{3}}{S_{0}^{k}} m_{k}, & K^{*}=\frac{L_{0}}{S_{0} U_{0}} K, & \boldsymbol{F}_{\boldsymbol{d}}^{*}=\frac{L_{0}}{U_{0}^{2}} \boldsymbol{F}_{\boldsymbol{d}} .
\end{array}
$$

Next, we take $\rho_{0}=\rho_{d}$ (the constant density of each droplet particle) to be the reference density, $T_{0}$ (the constant temperature of the spray) to be the reference temperature. Then, $\mu_{g, 0}=\mu_{g}\left(T_{0}\right)$ is the reference dynamic viscosity. Consider a second set of dimensionless quantities

$$
\begin{aligned}
& \rho_{g}^{*}=\frac{1}{\rho_{0}} \rho_{g}, \quad P_{g}^{*}=\frac{1}{\rho_{0} u_{0}^{2}} P_{g}, \quad \boldsymbol{u}_{\boldsymbol{g}}^{*}=\frac{1}{U_{0}} \boldsymbol{u}_{\boldsymbol{g}}, \quad E_{g}^{*}=\frac{1}{U_{0}^{2}} E_{g}, \\
& T_{g}^{*}=\frac{1}{T_{0}} T_{g}, \quad R_{g}^{*}=\frac{T_{0}}{U_{0}^{2}} R_{g}, \quad \mu_{g}^{*}=\frac{1}{\mu_{g, 0}} \mu_{g}, \quad \boldsymbol{\Sigma}_{g}^{*}=\frac{L_{0}^{2}}{\mu_{g, 0} U_{0}} \boldsymbol{\Sigma}_{\boldsymbol{g}} .
\end{aligned}
$$

Inserting (2.24) and (2.25) into (2.21)-(2.23) results in a non-dimensional version the EMSM model. This non-dimensional version consists of two parts.

- For the spray,

$$
\begin{array}{ccl}
\partial_{t^{*}}\left(m_{0}^{*}\right) & +\nabla_{\boldsymbol{x}^{*}} \cdot\left(m_{0}^{*} \boldsymbol{u}_{\boldsymbol{d}}^{*}\right) & =-K^{*} \tilde{n}^{*}\left(\boldsymbol{m}^{*}, S^{*}=0\right), \\
\partial_{t^{*}}\left(m_{1}^{*}\right) & +\nabla_{\boldsymbol{x}^{*}} \cdot\left(m_{1}^{*} \boldsymbol{u}_{\boldsymbol{d}}^{*}\right) & =-K^{*} m_{0}^{*}, \\
\partial_{t^{*}}\left(m_{2}^{*}\right) & +\nabla_{\boldsymbol{x}^{*}} \cdot\left(m_{2}^{*} \boldsymbol{u}_{\boldsymbol{d}}^{*}\right) & =-2 K^{*} m_{1}^{*}, \\
\vdots & \vdots & \vdots \\
\partial_{t^{*}}\left(m_{N}^{*}\right) & +\nabla_{\boldsymbol{x}^{*}} \cdot\left(m_{N}^{*} \boldsymbol{u}_{\boldsymbol{d}}^{*}\right) & =-N K^{*} m_{N-1}^{*}, \\
\partial_{t^{*}}\left(m_{1}^{*} \boldsymbol{u}_{\boldsymbol{d}}^{*}\right)+\nabla_{\boldsymbol{x}^{*}} \cdot\left(m_{1}^{*} \boldsymbol{u}_{\boldsymbol{d}}^{*} \otimes \boldsymbol{u}_{\boldsymbol{d}}^{*}\right)=-K^{*} m_{0}^{*} \boldsymbol{u}_{\boldsymbol{d}}^{*}+\mathrm{St}^{-1} \mu_{g}^{*} m_{0}^{*}\left(\boldsymbol{u}_{\boldsymbol{g}}^{*}-\boldsymbol{u}_{\boldsymbol{d}}^{*}\right),
\end{array}
$$


where

$$
\mathrm{St}=\frac{\rho_{0} S_{0} U_{0}}{18 \pi \mu_{g, 0} L_{0}}
$$

is the Stokes number. The last term in the right-hand side of (2.26e) can be expressed as $\int S^{*} \boldsymbol{F}_{\boldsymbol{d}}^{*} \tilde{n}^{*} \mathrm{~d} S^{*}$, which is consistent with the primary form $(2.13 \mathrm{~b})$. We have to be cautious, though, as to the value of the dimensionless drag force, viz.

$$
\boldsymbol{F}_{\boldsymbol{d}}^{*}=\mathrm{St}^{-1} \frac{\mu_{g}^{*}\left(\boldsymbol{u}_{\boldsymbol{g}}^{*}-\boldsymbol{u}_{\boldsymbol{d}}^{*}\right)}{S^{*}}
$$

which could not be directly guessed from the Stokes law (2.6).

- For the gas,

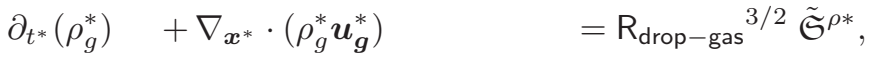

$$
\begin{aligned}
& \partial_{t^{*}}\left(\rho_{g}^{*} \boldsymbol{u}_{\boldsymbol{g}}^{*}\right)+\nabla_{\boldsymbol{x}^{*}} \cdot\left(\rho_{g}^{*} \boldsymbol{u}_{\boldsymbol{g}}^{*} \otimes \boldsymbol{u}_{\boldsymbol{g}}^{*}+P_{g}^{*} \boldsymbol{I}\right)=\mathrm{R}_{\mathrm{drop}-\text { gas }}{ }^{3 / 2} \tilde{\mathfrak{S}}^{\rho \boldsymbol{u} *}+\operatorname{Re}^{-1} \nabla_{\boldsymbol{x}^{*}} \cdot\left(\boldsymbol{\Sigma}_{\boldsymbol{g}}^{*}\right), \\
& \partial_{t^{*}}\left(\rho_{g}^{*} E_{g}^{*}\right)+\nabla_{\boldsymbol{x}^{*}} \cdot\left(\rho_{g}^{*} E_{g}^{*} \boldsymbol{u}_{\boldsymbol{g}}^{*}+P_{g}^{*} \boldsymbol{u}_{\boldsymbol{g}}^{*}\right)=\mathrm{R}_{\mathrm{drop}-\text { gas }}{ }^{3 / 2} \tilde{\mathfrak{S}}^{\rho e *}+\operatorname{Re}^{-1} \nabla_{\boldsymbol{x}^{*}} \cdot\left(\boldsymbol{\Sigma}_{\boldsymbol{g}}^{*} \cdot \boldsymbol{u}_{\boldsymbol{g}}^{*}\right) \text {, }
\end{aligned}
$$

where

$$
\operatorname{Re}=\frac{\rho_{0} U_{0} L_{0}}{\mu_{g, 0}} \quad \text { and } \quad \mathrm{R}_{\text {drop-gas }}=\frac{S_{0}}{L_{0}^{2}}=\frac{18 \pi \mathrm{St}}{\operatorname{Re}},
$$

are, respectively, the Reynolds number and a non-dimensional number arising due to the presence of two distinct reference surfaces for the spray and the gas. This number can be expressed as a combination between the Reynolds and Stokes numbers. The dimensionless source terms are

$$
\begin{aligned}
\tilde{\mathfrak{S}}^{\rho *} & =\frac{1}{4 \pi^{1 / 2}} K^{*} \tilde{m}_{1 / 2}^{*}, \\
\tilde{\mathfrak{S}}^{\rho \boldsymbol{u} *} & =\frac{1}{4 \pi^{1 / 2}} K^{*} \tilde{m}_{1 / 2}^{*} \boldsymbol{u}_{\boldsymbol{d}}^{*}-\frac{1}{6 \pi^{1 / 2}} \mathrm{St}^{-1} \mu_{g}^{*} \tilde{m}_{1 / 2}^{*}\left(\boldsymbol{u}_{\boldsymbol{g}}^{*}-\boldsymbol{u}_{\boldsymbol{d}}^{*}\right), \\
\tilde{\mathfrak{S}}^{\rho e *} & =\frac{1}{4 \pi^{1 / 2}} K^{*} \tilde{m}_{1 / 2}^{*} h_{d}^{*} .
\end{aligned}
$$

Again, the last term in the right-hand side of $(2.31 \mathrm{~b})$ can be expressed as $-\int V\left(S^{*}\right) \tilde{n}^{*} \boldsymbol{F}_{\boldsymbol{d}}^{*} \mathrm{~d} S^{*}$, which is consistent with the primary form $(2.8 \mathrm{~b})$.

It appears from the previous calculations that the EMSM model is governed essentially by two parameters: the Reynolds number and the Stokes number. For later use, we observe that the Stokes number (2.27) is the ratio of two characteristic times, that is,

$$
\text { St }=\frac{\tau_{d}}{\tau_{g, 0}}, \quad \text { with } \quad \tau_{d}=\frac{\rho_{0} S_{0}}{18 \pi \mu_{g, 0}} \quad \text { and } \quad \tau_{g, 0}=\frac{L_{0}}{U_{0}} .
$$

\subsection{Realizability of moments}

The EMSM model belongs to the class of high order moment methods, to the extent that it predicts the evolution of size moments of orders from 0 to $N$. In such a refined picture, our biggest concern is to keep the $(N+1)$-tuple $\boldsymbol{m}$ feasible in the following sense.

Given a domain $\mathfrak{D} \subset \mathbb{R}^{+}$, let $\mathfrak{M}_{N+1}$ be the set of those $\boldsymbol{m}=\left(m_{0}, m_{1}, \ldots, m_{N}\right)$ that coincide with the first $N+1$ moments of some positive-valued function $n$ defined on $\mathfrak{D}$, namely,

$$
m_{k}=\int_{\mathfrak{D}} S^{k} n(S) \mathrm{d} S, \quad \text { for all } k \in\{0,1, \ldots, N\} .
$$

Although $\mathfrak{M}_{N+1}$ is plainly a convex set, its geometrical shape is quite convoluted [16]. Characterizing $\mathfrak{M}_{N+1}$ by a set of necessary and sufficient algebraic conditions is called the Stieljes moment problem when $\mathfrak{D}=\mathbb{R}^{+}$, the Hausdorff moment problem ${ }^{6}$ when $\mathfrak{D}=\left[0, S_{0}\right]$. For a finite $N$, uniqueness of $n$ is no

\footnotetext{
${ }^{6}$ As a matter of fact, the Stieltjes and Hausdorff problems were originally stated for an infinite sequence $(N=\infty)$, for which it is also possible to recover uniqueness of the function $n$ under mild additional assumptions.
} 
longer guaranteed. This is not a real issue, since as seen earlier, we have the unique entropy maximization reconstruction [48] at our disposal. The real issues arise when evolution equations on $\boldsymbol{m}$ are imposed and solved by some approximation method.

- At the continuous level, the evolution operator applied to $\boldsymbol{m}$ need to be consistent with what we call the realizability condition. This means that if $\boldsymbol{m}(t=0) \in \mathfrak{M}_{N+1}$, then $\boldsymbol{m}(t) \in \mathfrak{M}_{N+1}$ for all $t>0$. The evolution operator on the size-moments in the present case is constituted of the sum of an evaporation operator, represented by the right-hand side of (2.21), and of a convection operator, represented by the divergence $\nabla_{\boldsymbol{x}}$. in the left-hand side of (2.21).

- At the discrete level, the numerical scheme used to update $\boldsymbol{m}$ need be consistent with the realizability condition. That is, if $\tilde{\boldsymbol{m}}(t) \in \mathfrak{M}_{N+1}$, then $\tilde{\boldsymbol{m}}(t+\Delta t) \in \mathfrak{M}_{N+1}$, where $\Delta t$ is the time-step. Note that the realizability condition may fail at the discrete level while fulfilled at the continuous level. In such a situation, the sequence $\tilde{\boldsymbol{m}}$ is said to be corrupted and this leads the immediate crash of the simulation or requires clipping and thus loss of computational efficiency as well as accuracy.

The challenge when using such moment methods is thus to come up with a numerical method satisfying the realizability condition. One can found in the literature tools to handle evaporation and convection of polydisperse spray using moment method EMSM, see [48] and [39], with high order time-space accuracy and consistency with $\mathfrak{M}_{N+1}$. We shall of course make use of these tools. In this context, we shall, in order to come up with a high-order convection scheme, work with quantities derived from the moments rather than with the moments themselves. To illustrate this point, let us consider a moment advection equation ${ }^{7}$

$$
\partial_{t} m_{k}+\nabla_{\boldsymbol{x}} \cdot\left(m_{k} \boldsymbol{w}\right)=0, \quad k \in\{0,1, \ldots, N\},
$$

and let us see how this equation evolves for normalized as well as canonical moments. In this illustration, the velocity field $\boldsymbol{w}$ can be either an imposed velocity field, or a velocity field resolved in a coupled manner with the vector of moments. In this pure transport of the moments, $\boldsymbol{m}^{n+1}$ is simply a shifted version of $\boldsymbol{m}^{n}$. Hence, $\boldsymbol{m}^{n+1} \in \mathfrak{M}_{N+1}$ if $\boldsymbol{m}^{n} \in \mathfrak{M}_{N+1}$, which is consistent with the realizability of moments at the continuous level. At the discrete level, however, things are more delicate. Indeed, if first-order standard finite volume schemes automatically preserve the moment space, increasing the order while preserving realizability is not straightforward. For second-order finite volume schemes using limited slope reconstructions, $\mathfrak{M}_{N+1}$ must be preserved in both the reconstruction step and fluxes computation. Wright [68] showed that, due to the complex shape of $\mathfrak{M}_{N+1}$, independent linear reconstruction of the moments may violate this requirement. To overcome the difficulty, Kah et al. [39] suggested to reconstruct not the moments but some derived quantities called canonical moments. To define these, let

$$
c_{0}=1 \quad \text { and } \quad c_{k}=\frac{m_{k}}{m_{0}}, \quad k \in\{1,2, \ldots, N\}
$$

be the normalized moments. From (2.34), it is straightforward to show that

$$
\partial_{t} c_{k}+\boldsymbol{w} \cdot \nabla_{\boldsymbol{x}} c_{k}=0
$$

Using the notation $\boldsymbol{c}_{k-1}=\left(c_{0}, c_{1}, \ldots, c_{k-1}\right)$ for $k \geq 1$, we define

$$
\wp\left(\boldsymbol{c}_{k-1}\right)=\left\{\left(c_{0}, c_{1}, \ldots, c_{k-1}, \gamma\right) \in \mathfrak{M}_{k+1} \text { for all possible } \gamma\right\}
$$

Dette and Studden [16] introduced the canonical moments of order $k$ as the ratio

$$
p_{k}=\frac{c_{k}-c_{k}^{-}\left(\boldsymbol{c}_{k-1}\right)}{c_{k}^{+}\left(\boldsymbol{c}_{k-1}\right)-c_{k}^{-}\left(\boldsymbol{c}_{k-1}\right)},
$$

\footnotetext{
${ }^{7}$ The stumbling difficulty of moment advection illustrated here will be encountered in the numerical method proposed in the present paper since it will be based on operator splitting.
} 
where

$$
c_{k}^{+}\left(\boldsymbol{c}_{k-1}\right)=\max _{\boldsymbol{\gamma} \in \wp\left(\boldsymbol{c}_{k-1}\right)} c_{k}(\boldsymbol{\gamma}) \quad \text { and } \quad c_{k}^{-}\left(\boldsymbol{c}_{k-1}\right)=\min _{\boldsymbol{\gamma} \in \wp\left(\boldsymbol{c}_{k-1}\right)} c_{k}(\boldsymbol{\gamma})
$$

are respectively the upper and lower bounds of the admissible interval for the last component, for the vector $\boldsymbol{c}_{k}=\left(c_{0}, c_{1}, \ldots, \gamma\right)$ to belong to $\mathfrak{M}_{k+1}$. From (2.36), it follows [39] that the canonical moments are advected too in the convection step, i.e.,

$$
\partial_{t} p_{k}+\boldsymbol{w} \cdot \nabla_{\boldsymbol{x}} p_{k}=0
$$

The decisive advantage of canonical moments is that the realizability condition amounts to

$$
p_{k} \in[0,1], \quad \text { for all } k \in\{1,2, \ldots, N\} \text {. }
$$

The geometry of this condition is very simple. As a result, it becomes possible to design [9] a slope limited reconstruction for the $p_{k}$ 's based on standard techniques. Details at the discrete level are given in $\S$ Appendix A.3. A numerical study is conducted in $\S 4.2$ to demonstrate the capabilities of the proposed scheme.

The present paper extends these techniques to simulate an injection occurring in internal combustion engines, using the industrial CFD code IFP-C3D, developed at IFPEN for automotive engine simulations. In this context, we will introduce two salient features: a two-way coupling and an original implicit method ensuring moment space conservation to handle it. We will come up with a discretization scheme that takes into account mesh movement and thus proposes a first ALE algorithm ensuring the realizability condition.

\section{Overview of the numerical method}

The EMSM model (2.21)-(2.23) is solved using a splitting strategy, where each stage corresponds to a class of physical phenomena. Instead of the standard Strang splitting, we rely on a splitting based on the Arbitrary Lagrange Euler (ALE) formalism [19, 34, 35, 24], which is more suitable to moving meshes. In both splitting approaches, the first stage - which we refer to as phase $A$ - is the same, since it is concerned with drag and evaporation source terms (as well as heat source in the gaseous phase equations due to evaporation). The discretization of these source terms for both carrier gas and spray gives rise to a local coupled system. A splitting strategy using explicit methods has been used in Doisneau et al. $[17,18]$ for lower order size-moments, whereas we propose an implicit method here for high order moments, which can be extended to arbitrary droplet models. This local system remains to be solved in a computationally efficient way.

We are going to describe this first stage with an emphasis on the two-way coupling, which is our novel contribution. Then, we will take a step back and look at the overall ALE formalism, which requires some work to be adapted to our two-velocity model.

\subsection{Two-way coupling source terms}

The system to be solved in phase $\mathrm{A}$ is obtained from (2.21)-(2.22) by dropping all divergence terms $\nabla_{\boldsymbol{x}} \cdot(\cdot)$. We thus have

$$
\begin{aligned}
\partial_{t}\left(m_{0}\right) & =-K \tilde{n}(\boldsymbol{m}, 0), \\
\partial_{t}\left(m_{1}\right) & =-K m_{0}, \\
\vdots & \vdots \\
\partial_{t}\left(m_{N}\right) & =-N K m_{N-1}, \\
\partial_{t}\left(m_{1} \boldsymbol{u}_{\boldsymbol{d}}\right) & =-K m_{0} \boldsymbol{u}_{\boldsymbol{d}}+\frac{18 \pi}{\rho_{d}} \mu_{g} m_{0}\left(\boldsymbol{u}_{\boldsymbol{g}}-\boldsymbol{u}_{\boldsymbol{d}}\right),
\end{aligned}
$$


for the spray, and

$$
\begin{aligned}
& \partial_{t}\left(\rho_{g}\right)=\tilde{\mathfrak{S}}^{\rho}, \\
& \partial_{t}\left(\rho_{g} \boldsymbol{u}_{\boldsymbol{g}}\right)=\tilde{\mathfrak{S}}^{\rho \boldsymbol{u}}, \\
& \partial_{t}\left(\rho_{g} E_{g}\right)=\tilde{\mathfrak{S}}^{\rho e},
\end{aligned}
$$

for the gas. In (3.1), we see that the first $N+1$ equations suffice to update $\boldsymbol{m}$, independently of the remaining equations.

The difficulty when attempting to solve system (3.1), is to preserve the realizability condition. At the discrete level, Massot et al. [48] have designed an evaporation solver that preserves the moment space. It relies on a kinetic scheme [54] coupled to a CQMOM approach [69]. The kinetic scheme allows to compute the source terms while ensuring realizability.

In fact, we use system (3.1)-(3.2) only for the one-way coupling model, i.e., when $\widetilde{\mathfrak{S}}^{\rho}=\tilde{\mathfrak{S}}^{\rho u}=\tilde{\mathfrak{S}}^{\rho e}=$ 0 . The fully-discrete details for the one-way coupling are recalled in Appendix A.1. For the two-way coupling model, we recommend two slight modifications of the system (3.1)-(3.2) in phase A. The purpose of these modifications is to make phase A more meaningful physically and easier to solve numerically.

1. On the grounds of (2.17b) and (2.19b), we replace (3.1d) by

$$
\partial_{t}\left(\tilde{m}_{3 / 2} \boldsymbol{u}_{\boldsymbol{d}}\right)=-\frac{3}{2} K \tilde{m}_{1 / 2} \boldsymbol{u}_{\boldsymbol{d}}+\frac{18 \pi}{\rho_{d}} \mu_{g} \tilde{m}_{1 / 2}\left(\boldsymbol{u}_{\boldsymbol{g}}-\boldsymbol{u}_{\boldsymbol{d}}\right),
$$

where $\tilde{m}_{k+1 / 2}=\int S^{k+1 / 2} \tilde{n}(\boldsymbol{m}, S) \mathrm{d} S$. This choice is driven by total momentum preservation $\partial_{t}\left(\rho_{g} \boldsymbol{u}_{\boldsymbol{g}}+\frac{1}{6 \pi^{1 / 2}} \rho_{d} \tilde{m}_{3 / 2} \boldsymbol{u}_{\boldsymbol{d}}\right)=0$.

2. In order to better separate the velocity $\boldsymbol{u}_{g}$ from other thermodynamical variables, we substitute to the total energy equation $(3.2 \mathrm{c})$ the internal energy equation

$$
\partial_{t}\left(\rho_{g} e_{g}\right)=\frac{\rho_{d}}{4 \pi^{1 / 2}} K \tilde{m}_{1 / 2} e_{d},
$$

where $e_{d}\left(T_{d}\right)=\int_{0}^{T_{d}} C_{v, d}(T) \mathrm{d} T$ is the spray internal energy. Within assumption (2.20), it is a constant and equal to $e_{d}\left(T_{d 0}\right)$. The gas internal energy is defined as $e_{g}=E_{g}-\frac{1}{2}\left\|\boldsymbol{u}_{g}\right\|^{2}$.

The phase A two-way coupling system now reads

$$
\begin{array}{ll}
\partial_{t}\left(m_{0}\right) & =-K \tilde{n}(\boldsymbol{m}, 0), \\
\partial_{t}\left(m_{1}\right) & =-K m_{0}, \\
\vdots & \vdots \\
\partial_{t}\left(m_{N}\right) & =-N K m_{N-1}, \\
\partial_{t}\left(\tilde{m}_{3 / 2} \boldsymbol{u}_{\boldsymbol{d}}\right) & =\underbrace{-\frac{3}{2} K \tilde{m}_{1 / 2} \boldsymbol{u}_{\boldsymbol{d}}}_{\text {evap. }}+\underbrace{\underbrace{18 \pi}_{\rho_{d}} \mu_{g} \tilde{m}_{1 / 2}\left(\boldsymbol{u}_{\boldsymbol{g}}-\boldsymbol{u}_{\boldsymbol{d}}\right.}_{\text {drag }})
\end{array}
$$

for the spray, and

$$
\begin{aligned}
& \partial_{t}\left(\rho_{g}\right)=\frac{1}{4 \pi^{1 / 2}} \rho_{d} K \tilde{m}_{1 / 2}, \\
& \partial_{t}\left(\rho_{g} \boldsymbol{u}_{\boldsymbol{g}}\right)=\frac{1}{4 \pi^{1 / 2}} \rho_{d} K \tilde{m}_{1 / 2} \boldsymbol{u}_{\boldsymbol{d}}-\underbrace{\frac{18 \pi}{6 \pi^{1 / 2}} \mu_{g} \tilde{m}_{1 / 2}\left(\boldsymbol{u}_{\boldsymbol{g}}-\boldsymbol{u}_{\boldsymbol{d}}\right)}_{\text {evap. }}, \\
& \partial_{t}\left(\rho_{g} e_{g}\right)=\underbrace{\frac{1}{4 \pi^{1 / 2}} \rho_{d} K \tilde{m}_{1 / 2} e_{d}}_{\text {drag }}, \underbrace{\underbrace{2}},
\end{aligned}
$$

for the gas. Let us assume, in addition to hypotheses (2.11), that $K$ does not depend on $\boldsymbol{u}_{\boldsymbol{d}}$-but may depend on $t$, as considered by Duarte et al. [20]. This holds true within the $d^{2}$ type evaporation law in the present work. Then, the first $N+1$ equations of (3.5) are decoupled from the remaining equations. As 
mentioned earlier and after Massot et al. [48], this subsystem (3.5a)-(3.5c) can be solved for $\boldsymbol{m}(t)$, from which we deduce $\tilde{m}_{1 / 2}(t)$ and $\tilde{m}_{3 / 2}(t)$. Plugging the latter into (3.6) yields $\rho_{g}$ and $\rho_{g} E_{g}$ as an explicit function of time, that is,

$$
\begin{aligned}
\left(\rho_{g}\right)(t) & =\left(\rho_{g}\right)^{n}+\frac{1}{4 \pi^{1 / 2}} \rho_{d} \int_{t^{n}}^{t} K(\tau) \tilde{m}_{1 / 2}(\tau) \mathrm{d} \tau \\
\left(\rho_{g} e_{g}\right)(t) & =\left(\rho_{g} e_{g}\right)^{n}+\frac{1}{4 \pi^{1 / 2}} \rho_{d} \int_{t^{n}}^{t} K(\tau) \tilde{m}_{1 / 2}(\tau) e_{d} \mathrm{~d} \tau
\end{aligned}
$$

The two remaining equations of (3.5)-(3.6), i.e.,

$$
\begin{aligned}
& \partial_{t}\left(\tilde{m}_{3 / 2} \boldsymbol{u}_{\boldsymbol{d}}\right)=-\frac{3}{2} K \tilde{m}_{1 / 2} \boldsymbol{u}_{\boldsymbol{d}}+\frac{18 \pi}{\rho_{d}} \mu_{g} \tilde{m}_{1 / 2}\left(\boldsymbol{u}_{\boldsymbol{g}}-\boldsymbol{u}_{\boldsymbol{d}}\right) \\
& \partial_{t}\left(\rho_{g} \boldsymbol{u}_{\boldsymbol{g}}\right)=\frac{\rho_{d}}{4 \pi^{1 / 2}} K \tilde{m}_{1 / 2} \boldsymbol{u}_{\boldsymbol{d}}-\frac{18 \pi}{6 \pi^{1 / 2}} \mu_{g} \tilde{m}_{1 / 2}\left(\boldsymbol{u}_{\boldsymbol{g}}-\boldsymbol{u}_{\boldsymbol{d}}\right)
\end{aligned}
$$

are strongly coupled. Setting $\boldsymbol{y}=\left(\boldsymbol{u}_{\boldsymbol{d}}, \boldsymbol{u}_{\boldsymbol{g}}\right)$, the above system can be put under the abstract form

$$
\mathrm{d}_{t} \boldsymbol{y}=\boldsymbol{g}(\boldsymbol{y}, t)
$$

where the explicit dependency of $\boldsymbol{g}$ in $t$ reflects our knowledge of $\tilde{m}_{1 / 2}(t), \tilde{m}_{3 / 2}(t)$ and $\rho_{g}(t), e_{g}(t)$. As for $\mu_{g}=\mu_{g}\left(T_{g}\right)$, it can also be viewed as a function $t$ via $T_{g}=e_{g} / C_{v, g}=\left(\gamma_{g}-1\right) e_{g} / R_{g}$.

Taking into account the space discretization over a staggered grid requires some attention. For the sake of clarity, let us write down the details for the 1-D case. Generalization to the 3-D case is straightforward. Throughout phase A, since no mesh movement occurs, the spray $\boldsymbol{x}$-domain is divided into static disjoint cells

of length

$$
\left[x_{i-1 / 2}(t), x_{i+1 / 2}(t)\left[=\left[x_{i-1 / 2}^{n}, x_{i+1 / 2}^{n}[,\right.\right.\right.
$$

$$
\Delta x_{i}(t):=x_{i+1 / 2}(t)-x_{i-1 / 2}(t)=x_{i+1 / 2}^{n}-x_{i-1 / 2}^{n}=\Delta x_{i}^{n} .
$$

At the centers $x_{i}=\frac{1}{2}\left(x_{i-1 / 2}+x_{i+1 / 2}\right)$ of these primal cells, we seek approximate values for the moments $\boldsymbol{m}_{i}$, the gas density $\rho_{g, i}$ and the total energy $E_{g, i}$. Solving the ODE sub-sytems (3.5a)-(3.5c) and (3.6a), (3.6c) on each primal cell, we have $\boldsymbol{m}_{i}(t), \rho_{g, i}(t)$ and $\left(\rho_{g} e_{g}\right)_{i}(t)$ by formulae (3.7).

The dual cells are defined as $\left[x_{i}^{n}, x_{i+1}^{n}\left[\right.\right.$ and are assigned the velocities $\boldsymbol{u}_{\boldsymbol{d} i+1 / 2}$ and $\boldsymbol{u}_{\boldsymbol{g}_{i+1 / 2}}$. In conformity with the finite-volume spirit, we first integrate (3.8) over the dual cell $\left[x_{i}, x_{i+1}\right.$ [ to get

$$
\begin{aligned}
& \mathrm{d}_{t} \int_{x_{i}}^{x_{i+1}} \tilde{m}_{3 / 2} \boldsymbol{u}_{\boldsymbol{d}} \mathrm{d} x=-\frac{3}{2} \int_{x_{i}}^{x_{i+1}} K \tilde{m}_{1 / 2} \boldsymbol{u}_{\boldsymbol{d}} \mathrm{d} x+\frac{18 \pi}{\rho_{d}} \int_{x_{i}}^{x_{i+1}} \mu_{g} \tilde{m}_{1 / 2}\left(\boldsymbol{u}_{\boldsymbol{g}}-\boldsymbol{u}_{\boldsymbol{d}}\right) \mathrm{d} x \\
& \mathrm{~d}_{t} \int_{x_{i}}^{x_{i+1}} \rho_{g} \boldsymbol{u}_{\boldsymbol{g}} \mathrm{d} x=\frac{\rho_{d}}{4 \pi^{1 / 2}} \int_{x_{i}}^{x_{i+1}} K \tilde{m}_{1 / 2} \boldsymbol{u}_{\boldsymbol{d}} \mathrm{d} x-\frac{18 \pi}{6 \pi^{1 / 2}} \int_{x_{i}}^{x_{i+1}} \mu_{g} \tilde{m}_{1 / 2}\left(\boldsymbol{u}_{\boldsymbol{g}}-\boldsymbol{u}_{\boldsymbol{d}}\right) \mathrm{d} x
\end{aligned}
$$

The next step is to invoke the approximations

$$
\begin{aligned}
\int_{x_{i}}^{x_{i+1}} \tilde{m}_{3 / 2} \boldsymbol{u}_{\boldsymbol{d}} \mathrm{d} x & \approx \frac{1}{2}\left(\Delta x_{i} \tilde{m}_{3 / 2, i}+\Delta x_{i+1} \tilde{m}_{3 / 2, i+1}\right) \boldsymbol{u}_{\boldsymbol{d} i+1 / 2}, \\
\int_{x_{i}}^{x_{i+1}} \rho_{g} \boldsymbol{u}_{\boldsymbol{g}} \mathrm{d} x & \approx \frac{1}{2}\left(\Delta x_{i} \rho_{g, i}+\Delta x_{i+1} \rho_{g, i+1}\right) \boldsymbol{u}_{\boldsymbol{g}_{i+1 / 2}}, \\
\int_{x_{i}}^{x_{i+1}} K \tilde{m}_{1 / 2} \boldsymbol{u}_{\boldsymbol{d}} \mathrm{d} x & \approx \frac{1}{2}\left(\Delta x_{i} K_{i} \tilde{m}_{1 / 2, i}+\Delta x_{i+1} K_{i+1} \tilde{m}_{1 / 2, i+1}\right) \boldsymbol{u}_{\boldsymbol{d} i+1 / 2}, \\
\int_{x_{i}}^{x_{i+1}} \mu_{g} \tilde{m}_{1 / 2}\left(\boldsymbol{u}_{\boldsymbol{g}}-\boldsymbol{u}_{\boldsymbol{d}}\right) \mathrm{d} x & \approx \frac{1}{2}\left(\Delta x_{i} \mu_{g, i} \tilde{m}_{1 / 2, i}+\Delta x_{i+1} \mu_{g, i+1} \tilde{m}_{1 / 2, i+1}\right)\left(\boldsymbol{u}_{\boldsymbol{g}}-\boldsymbol{u}_{\boldsymbol{d}}\right)_{i+1 / 2},
\end{aligned}
$$

in order to obtain an ODE system of the form (3.9). This ODE system is solved by the Singly Diagonally Implicit Runge-Kutta Method (SDIRK) given by the Butcher array [33]

$$
\begin{array}{c|ccc}
\omega & \omega & & \\
\frac{1}{2}(1+\omega) & \frac{1}{2}(1-\omega) & \omega & \\
1 & \frac{1}{4}\left(-1+16 \omega-6 \omega^{2}\right) & \frac{1}{4}\left(5-20 \omega+6 \omega^{2}\right) & \omega \\
\hline & \frac{1}{4}\left(-1+16 \omega-6 \omega^{2}\right) & \frac{1}{4}\left(5-20 \omega+6 \omega^{2}\right) & \omega
\end{array},
$$

with $\omega=0.4358665215$ being a root of $6 \omega^{3}-18 \omega^{2}+9 \omega-1=0$. This third-order numerical integration scheme is relevant to our problem. For one, it is simple and inexpensive in the context of industrial 
software development. For another, it provides the A-stability and L-stability ${ }^{8}$ properties. These will be essential when using more sophisticated droplet models such as Schiller and Nauman's drag model [58], where the ODE becomes nonlinear and the coupling is stiffer.

\subsection{Arbitrary Lagrange Euler formalism}

In contrast to previous EMSM-related works $[48,39]$ where calculations were settled on fixed meshes, the realistic configurations considered in our case - injection in combustion engines with a moving pistoncommand to carry out computations over moving meshes. The most common technique to cope with this new constraint is the Arbitrary Lagrange Euler (ALE) formalism, which was introduced [34] in the context of single-velocity fluid flows. Let us see how to adapt the ALE philosophy to the EMSM model, in which two velocity fields co-exist.

We introduce a new referential frame, attached to the grid points, in which the coordinates are denoted by $\chi$. This frame is, a priori, neither the material (Lagrangian) configuration nor the laboratory (Eulerian) configuration $\boldsymbol{x}$. Instead, it moves at the imposed velocity $\boldsymbol{w}$ with respect to the laboratory. Let $\boldsymbol{x}=\boldsymbol{x}(t, \boldsymbol{\chi})$ be the correspondence between the moving frame and the laboratory frame. This correspondence is determined by

$$
\partial_{t} \boldsymbol{x}=\boldsymbol{w} .
$$

Let $J_{d}=\operatorname{det}\left(\nabla_{\chi} x\right)$ be the dilatation rate. Then, it is a classical result that $J_{d}$ evolves in time according to

$$
\partial_{t} J_{d}=J_{d} \nabla_{\boldsymbol{x}} \cdot \boldsymbol{w} .
$$

In the ALE formalism, $J_{d}$ will act as a new scalar unknown subject to (3.13). To properly account for two-velocity mixtures, we propose to copy $J_{d}$ into a second scalar unknown $J_{g}$, governed by

$$
\partial_{t} J_{g}=J_{g} \nabla_{\boldsymbol{x}} \cdot \boldsymbol{w}
$$

The idea is then to consider $J_{d}$ and $J_{g}$ as two independent fields, since each phase has its own velocity. We are now ready to perform a change of variables from $(t, \boldsymbol{x})$ to $(t, \boldsymbol{\chi})$. Standard calculations [15] show that system (2.21)-(2.22) is equivalent to the following problem.

- For the spray, find $\left(J_{d}, \boldsymbol{m}, \boldsymbol{u}_{\boldsymbol{d}}\right)=\left(J_{d}, m_{0}, m_{1}, \ldots, m_{N}, \boldsymbol{u}_{\boldsymbol{d}}\right)$ such that

$$
\begin{aligned}
& \partial_{t}\left(J_{d}\right) \quad+J_{d} \nabla_{\boldsymbol{x}} \cdot\left(\boldsymbol{u}_{\boldsymbol{d}}-\boldsymbol{w}\right) \quad-J \nabla_{\boldsymbol{x}} \cdot\left(\boldsymbol{u}_{\boldsymbol{d}}\right)=0, \\
& \partial_{t}\left(J_{d} m_{0}\right) \quad+J_{d} \nabla_{\boldsymbol{x}} \cdot\left(m_{0}\left(\boldsymbol{u}_{\boldsymbol{d}}-\boldsymbol{w}\right)\right) \quad=-K J_{d} \tilde{n}(\boldsymbol{m}, S=0), \\
& \partial_{t}\left(J_{d} m_{1}\right) \quad+J_{d} \nabla_{\boldsymbol{x}} \cdot\left(m_{1}\left(\boldsymbol{u}_{\boldsymbol{d}}-\boldsymbol{w}\right)\right) \quad=-K J_{d} m_{0}, \\
& \vdots \quad \vdots \quad \vdots \\
& \partial_{t}\left(J_{d} m_{N}\right)+J_{d} \nabla_{\boldsymbol{x}} \cdot\left(m_{N}\left(\boldsymbol{u}_{\boldsymbol{d}}-\boldsymbol{w}\right)\right) \quad=-N K J_{d} m_{N-1}, \\
& \partial_{t}\left(J_{d} m_{1} \boldsymbol{u}_{\boldsymbol{d}}\right)+\underbrace{J_{d} \nabla_{\boldsymbol{x}} \cdot\left(m_{1} \boldsymbol{u}_{\boldsymbol{d}} \otimes\left(\boldsymbol{u}_{\boldsymbol{d}}-\boldsymbol{w}\right)\right)} \underbrace{\longrightarrow}=-K \underbrace{J_{d} m_{0} \boldsymbol{u}_{\boldsymbol{d}}+\frac{18 \pi}{\rho} J_{d} \mu_{g} m_{0}\left(\boldsymbol{u}_{\boldsymbol{g}}-\boldsymbol{u}_{\boldsymbol{d}}\right)} .
\end{aligned}
$$

$$
\text { C B } \quad \text { A }
$$

- For the gas, find $\left(J_{g}, \rho_{g}, \boldsymbol{u}_{\boldsymbol{g}}, E_{g}\right)$ such that

$$
\begin{array}{lll}
\partial_{t}\left(J_{g}\right)+J_{g} \nabla_{\boldsymbol{x}} \cdot\left(\left(\boldsymbol{u}_{\boldsymbol{g}}-\boldsymbol{w}\right)\right) & -J_{g} \nabla_{\boldsymbol{x}} \cdot\left(\boldsymbol{u}_{\boldsymbol{g}}\right) & =0, \\
\partial_{t}\left(J_{g} \rho_{g}\right)+J_{g} \nabla_{\boldsymbol{x}} \cdot\left(\rho_{g}\left(\boldsymbol{u}_{\boldsymbol{g}}-\boldsymbol{w}\right)\right) & =J_{g} \tilde{\mathfrak{S}}^{\rho}, \\
\partial_{t}\left(J_{g} \rho_{g} \boldsymbol{u}_{\boldsymbol{g}}\right)+J_{g} \nabla_{\boldsymbol{x}} \cdot\left(\rho_{g} \boldsymbol{u}_{\boldsymbol{g}} \otimes\left(\boldsymbol{u}_{\boldsymbol{g}}-\boldsymbol{w}\right)\right) & +J_{g} \nabla_{\boldsymbol{x}} \cdot\left(P_{g} \boldsymbol{I}-\boldsymbol{\Sigma}_{\boldsymbol{g}}\right) & =J_{g} \tilde{\mathfrak{S}}^{\rho \boldsymbol{u}}, \\
\partial_{t}\left(J_{g} \rho_{g} E_{g}\right)+\underbrace{J_{g} \nabla_{\boldsymbol{x}} \cdot\left(\rho_{g} E_{g}\left(\boldsymbol{u}_{\boldsymbol{g}}-\boldsymbol{w}\right)\right)}_{\mathrm{C}}+\underbrace{J_{g} \nabla_{\boldsymbol{x}} \cdot\left(\left(P_{g} \boldsymbol{I}-\boldsymbol{\Sigma}_{\boldsymbol{g}}\right) \cdot \boldsymbol{u}_{\boldsymbol{g}}\right)}_{\mathrm{B}} & =\underbrace{J_{g} \tilde{\mathfrak{S}}^{\rho e}}_{\mathrm{A}}
\end{array}
$$

\footnotetext{
${ }^{8}$ The solution of the ODE $d y / d t=\lambda y$, with $\operatorname{Re}(\lambda)<0$, is stable in the sense that $y(t) \rightarrow 0$ as $t \rightarrow+\infty$. A numerical scheme $y_{n+1}=R(\lambda \Delta t) y_{n}$ is said to be A-stable if it reproduces this decay property, i.e., if $|R(z)|<1$ for all $\operatorname{Re}(z)<0$. Furthermore, the method is said to be L-stable if stiff modes are highly damped, that is, if $R(z) \rightarrow 0$ as $\operatorname{Re}(z) \rightarrow-\infty$.
} 
As earlier, $\left(\tilde{\mathfrak{S}}^{\rho}, \tilde{\mathfrak{S}}^{\rho \boldsymbol{u}}, \tilde{\mathfrak{S}}^{\rho e}\right)$ are either zero (one-way coupling) or given by (2.23) (two-way coupling). The unknowns

$$
\mathbb{U}_{d}=\left(J_{d}, \boldsymbol{m}, \boldsymbol{u}_{\boldsymbol{d}}\right) \quad \text { and } \quad \mathbb{U}_{g}=\left(J_{g}, \rho_{g}, \boldsymbol{u}_{\boldsymbol{g}}, E_{g}\right),
$$

must be viewed as functions of $(t, \chi)$. In practice, we never have to handle the space variable $\chi$, since by appropriate integration over control volumes in the $(t, \chi)$-frame, we can bring every thing back to the $(t, \boldsymbol{x})$-frame. Besides, we insist that although $J_{d}$ and $J_{g}$ remain identical in the unsplit system, they should be regarded as two distinct quantities. This viewpoint will be useful for the splitting procedure, in the course of which $J_{d}$ may differ from $J_{g}$.

\subsection{Elements of time-space discretization}

To update the variables of (3.15)-(3.16) from time $t^{n}$ to $t^{n+1}=t^{n}+\Delta t$, the algorithm goes through 3 stages, or phases, traditionally called A, B and C. This is depicted by the diagram

$$
\left(\mathbb{U}_{d}^{n}, \mathbb{U}_{g}^{n}\right) \underset{\Delta t}{\stackrel{\mathrm{A}}{\longrightarrow}}\left(\mathbb{U}_{d}^{A}, \mathbb{U}_{g}^{A}\right) \underset{\Delta t}{\stackrel{\mathrm{B}}{\longrightarrow}}\left(\mathbb{U}_{d}^{B}, \mathbb{U}_{g}^{B}\right) \underset{\Delta t}{\stackrel{\mathrm{C}}{\longrightarrow}}\left(\mathbb{U}_{d}^{n+1}, \mathbb{U}_{g}^{n+1}\right) .
$$

Far from being a mere algebraic decomposition, each stage does have a most natural physical meaning. In this respect, the ALE formalism is powerful even for a fixed mesh $(\boldsymbol{w}=0)$. Starting from $J_{d}^{n}=J_{g}^{n}$, one will generally have

$$
J_{d}^{A}=J_{g}^{A}, \quad J_{d}^{B} \neq J_{g}^{B}, \quad J_{d}^{n+1}=J_{g}^{n+1} .
$$

To gain more insight into this splitting strategy, let us review some features of each phase.

In phase $\mathrm{A}$, since $\partial_{t} J_{d}=\partial_{t} J_{g}=0$, the dilatation rates $J_{d}$ and $J_{g}$ can be simplified from the equations. These boil down to (3.1)-(3.2) and can be solved as explained in $\S 3.1$ for the two-way coupling and Appendix A.1 for the one-way coupling.

In phase B, we have to deal with two uncoupled systems, namely,

$$
\begin{aligned}
& \partial_{t}\left(J_{d}\right) \quad-J_{d} \nabla_{\boldsymbol{x}} \cdot\left(\boldsymbol{u}_{\boldsymbol{d}}\right)=0, \\
& \partial_{t}\left(J_{d} \boldsymbol{m}\right) \quad=0, \\
& \partial_{t}\left(J_{d} m_{1} \boldsymbol{u}_{\boldsymbol{d}}\right) \quad=0 \text {, }
\end{aligned}
$$

for the spray, and

$$
\begin{array}{ll}
\partial_{t}\left(J_{g}\right)-J_{g} \nabla_{\boldsymbol{x}} \cdot\left(\boldsymbol{u}_{\boldsymbol{g}}\right) & =0, \\
\partial_{t}\left(J_{g} \rho_{g}\right) & =0, \\
\partial_{t}\left(J_{g} \rho_{g} \boldsymbol{u}_{\boldsymbol{g}}\right)+J_{g} \nabla_{\boldsymbol{x}} \cdot\left(P_{g} \boldsymbol{I}-\boldsymbol{\Sigma}_{\boldsymbol{g}}\right) & =0, \\
\partial_{t}\left(J_{g} \rho_{g} E_{g}\right)+J_{g} \nabla_{\boldsymbol{x}} \cdot\left(\left(P_{g} \boldsymbol{I}-\boldsymbol{\Sigma}_{\boldsymbol{g}}\right) \cdot \boldsymbol{u}_{\boldsymbol{g}}\right) & =0,
\end{array}
$$

for the gas. We refer the readers to Appendix A.2 for the full space-time discretization of the spray system (3.20). Here, we highlight some of its properties at the continuous level. First, note that the intermediate equations $(3.20 \mathrm{~b})$ can be readily solved to give

$$
\boldsymbol{m}^{B}=\frac{J_{d}^{A}}{J_{d}^{B}} \boldsymbol{m}^{A},
$$

which implies that $\boldsymbol{m}^{B} \in \mathfrak{M}_{N+1}$ as soon as $\boldsymbol{m}^{A} \in \mathfrak{M}_{N+1}, J_{d}^{A}>0$ and provided that $J_{d}^{B}>0$. At the continuous level, phase B seems to be consistent with the realizability of moments. But the real difficulty lies in the fact that the hyperbolic system (3.20) is resonant. As a matter of fact, any subsystem

$$
\begin{array}{ll}
\partial_{t}\left(J_{d}\right)-J_{d} \nabla_{\boldsymbol{x}} \cdot\left(\boldsymbol{u}_{\boldsymbol{d}}\right) & =0, \\
\partial_{t}\left(J_{d} m_{k}\right) & =0, \\
\partial_{t}\left(\boldsymbol{u}_{\boldsymbol{d}}\right) & =0,
\end{array}
$$


extracted from (3.20), can be interpreted as the Lagrangian version of the pressureless gas system

$$
\begin{aligned}
& \partial_{t}\left(m_{k}\right)+\nabla_{\boldsymbol{x}} \cdot\left(m_{k} \boldsymbol{u}_{\boldsymbol{d}}\right)=0, \\
& \partial_{t}\left(m_{k} \boldsymbol{u}_{\boldsymbol{d}}\right)+\nabla_{\boldsymbol{x}} \cdot\left(m_{k} \boldsymbol{u}_{\boldsymbol{d}} \otimes \boldsymbol{u}_{\boldsymbol{d}}\right)=0 .
\end{aligned}
$$

The mathematical properties of pressureless gas dynamics have been investigated by many authors, notably by Bouchut $[6,7]$. Since no pressure term acts on the velocity field to prevent particle accumulation, high density moving concentrations called $\delta$-shocks are expected to develop. This behavior is due to the mono-kinetic assumption made on the spray velocity field in part 2, where we choose a Dirac delta function for the spray velocity distribution.

As far as the gas system (3.21) is concerned, it is none other than the Navier-Stokes gas dynamics in Lagrangian coordinates. The physics contained in this Lagrangian setting is essentially acoustics. Several numerical schemes are available for (3.21). We have chosen to rely on the one implemented in IFP-C3D. Details at the fully discrete level can be found in the KIVA report by Amsden et al. [2] or a relaxation variant by Coquel et al. [14].

In phase $\mathrm{C}$, we also have two uncoupled systems, namely,

$$
\begin{array}{lll}
\partial_{t}\left(J_{d}\right) & +J_{d} \nabla_{\boldsymbol{x}} \cdot\left(\boldsymbol{u}_{\boldsymbol{d}}-\boldsymbol{w}\right) & =0, \\
\partial_{t}\left(J_{d} m_{0}\right) & +J_{d} \nabla_{\boldsymbol{x}} \cdot\left(m_{0}\left(\boldsymbol{u}_{\boldsymbol{d}}-\boldsymbol{w}\right)\right) & =0, \\
\partial_{t}\left(J_{d} m_{1}\right) & +J_{d} \nabla_{\boldsymbol{x}} \cdot\left(m_{1}\left(\boldsymbol{u}_{\boldsymbol{d}}-\boldsymbol{w}\right)\right) & =0, \\
\vdots & \vdots & \vdots \\
\partial_{t}\left(J_{d} m_{N}\right) & +J_{d} \nabla_{\boldsymbol{x}} \cdot\left(m_{N}\left(\boldsymbol{u}_{\boldsymbol{d}}-\boldsymbol{w}\right)\right) & =0, \\
\partial_{t}\left(J_{d} m_{1} \boldsymbol{u}_{\boldsymbol{d}}\right) & +J_{d} \nabla_{\boldsymbol{x}} \cdot\left(m_{1} \boldsymbol{u}_{\boldsymbol{d}} \otimes\left(\boldsymbol{u}_{\boldsymbol{d}}-\boldsymbol{w}\right)\right) & =0,
\end{array}
$$

for the spray, and

$$
\begin{array}{lll}
\partial_{t}\left(J_{g}\right) & +J_{g} \nabla_{\boldsymbol{x}} \cdot\left(\left(\boldsymbol{u}_{\boldsymbol{g}}-\boldsymbol{w}\right)\right) & =0, \\
\partial_{t}\left(J_{g} \rho_{g}\right) & +J_{g} \nabla_{\boldsymbol{x}} \cdot\left(\rho_{g}\left(\boldsymbol{u}_{\boldsymbol{g}}-\boldsymbol{w}\right)\right) & =0, \\
\partial_{t}\left(J_{g} \rho_{g} \boldsymbol{u}_{\boldsymbol{g}}\right)+J_{g} \nabla_{\boldsymbol{x}} \cdot\left(\rho_{g} \boldsymbol{u}_{\boldsymbol{g}} \otimes\left(\boldsymbol{u}_{\boldsymbol{g}}-\boldsymbol{w}\right)\right) & =0, \\
\partial_{t}\left(J_{g} \rho_{g} E_{g}\right)+J_{g} \nabla_{\boldsymbol{x}} \cdot\left(\rho_{g} E_{g}\left(\boldsymbol{u}_{\boldsymbol{g}}-\boldsymbol{w}\right)\right) & =0,
\end{array}
$$

for the gas. We refer the readers to Appendix A.3 for the full space-time discretization of the spray system (3.25). To meet the challenge of preserving the realizability condition $\boldsymbol{m}^{n+1} \in \mathfrak{M}_{N+1}$, we follow Kah et al. [39].

Equations (3.26) of the gas system imply the pure advection of $\left(\rho_{g}, \rho_{g} u_{g}, \rho_{g} E_{g}\right)$ at velocity $\boldsymbol{u}_{\boldsymbol{g}}-\boldsymbol{w}$ by means of similar calculations. Here, the challenge is to preserve positivity for the density $\rho_{g}$ and the internal energy $e_{g}$. Again, first-order schemes meet the requirements but are not accurate enough. Higher order schemes have to equipped with additional treatment. The problem is all the trickier as IFP-C3D uses a staggered grid. The readers are referred to [5] for more details.

\section{Verification of two claimed features for the new EMSM}

As this work aims at extending two previous contributions [48, 39], we focus here on the illustration of EMSM two new capabilities, two-way coupling and moment transport in the ALE context, by means of some simple test cases. The test cases we consider below are directly inspired from those of [48, 39]. The numerical values assigned to various quantities have been calibrated so as to be representative of the upcoming injection calculations.

\subsection{Source terms for two-way coupling}

In order to test the method for phase A developed in $\S 3.1$, we concentrate on 0-D cases; phases B and $\mathrm{C}$ have been deactivated and analytical solutions can be found. In the two following test cases, the 
initial data (at $t=0)$ share the common values

$$
\begin{array}{llll}
\rho_{d}=760 \mathrm{~kg} / \mathrm{m}^{3}, & u_{d}=-1 \mathrm{~m} / \mathrm{s}, & T_{d}=293 \mathrm{~K}, & \mu_{g}=1.99 \cdot 10^{-5} \mathrm{~kg} / \mathrm{m} / \mathrm{s}, \\
\rho_{g}=1 \mathrm{~kg} / \mathrm{m}^{3}, & u_{g}=1 \mathrm{~m} / \mathrm{s}, & T_{g}=293 \mathrm{~K} . &
\end{array}
$$

Since our ultimate goal is to provide a Eulerian alternative for ICE applications, it would be meaningful to make an analogy between the $0-\mathrm{D}$ cases here and the $2-\mathrm{D}$ injection cases of $\S 6$. To put it another way, it would be nice to have comparable dimensionless numbers, but the problem of defining a reference length for a $0-\mathrm{D}$ case arises. We have chosen to take the injector diameter $L_{0}=8 \cdot 10^{-3} \mathrm{~m}$. We set $U_{0}=u_{g}(t=0)=1 \mathrm{~m} / \mathrm{s}$ for the reference speed and assume that the viscosity $\mu_{g}\left(T_{g}\right)$ is a constant $\mu_{g, 0}$ that does not depend on $T_{g}$. In addition to the Reynolds and Stokes numbers, it is also customary to define

$$
\Phi_{\mathrm{v}}=\frac{1}{6 \pi^{1 / 2}} m_{3 / 2}, \quad \Phi_{\mathrm{m}}=\frac{1}{6 \pi^{1 / 2}} \frac{\rho_{d}}{\rho_{g}} m_{3 / 2},
$$

as respectively the volume fraction and the mass loading. In terms of dimensionless quantities, the initial data (4.1) and the initial value of $m_{3 / 2}$ have been prepared so that

$$
\operatorname{Re}=3.05 \cdot 10^{5}, \quad \Phi_{\mathrm{v}}=1.31 \cdot 10^{-3}, \quad \Phi_{\mathrm{m}}=1 .
$$

This implies

$$
m_{3 / 2}(t=0)=1.4 \cdot 10^{-2} \text {. }
$$

Let us now focus on the calculation of the Stokes number St of the two-phase flow. From (2.32), the gas-phase characteristic time scale is calculated as $\tau_{g, 0}=8 \cdot 10^{-3} \mathrm{~s}$. The characteristic time scale $\tau_{d}$ associated to polydisperse spray dynamics is in function of the reference size $S_{0}$ and defined as

$$
S_{0}=\frac{m_{3 / 2}(t=0)}{m_{1 / 2}(t=0)},
$$

which is equal to the initial mean size of the polydisperse distribution, also converts to a reference radius expressed as $r_{0}=\left(S_{0} / 4 \pi\right)^{1 / 2}$. This choice for $S_{0}$ is particularly important since the drag dynamics are driven through the mean size of the polydisperse distribution, as deduced from Eq.(3.5d).

In the following, one will work with different size distributions, with $S \in\left[0, S_{\max }\right]$ and $S_{\max }=8.04$. $10^{-8} \mathrm{~m}^{2}$. This implies different $S_{0}$ and St for each distribution.

\subsubsection{Drag force alone}

The first numerical experiment, with $K=0$, is set up to assess numerical stability. As no evaporation comes in to change the size distribution, it is justified to consider a constant distribution in size of droplets

$$
n(t=0, S)=n_{0} \mathbf{1}_{\left[0, S_{\max }\right]}(S),
$$

where $n_{0}$ is chosen so that $m_{3 / 2}(t=0)=\int_{\mathbb{R}^{+}} S^{3 / 2} n(t=0, S) \mathrm{d} S=\frac{2}{5} n_{0} S_{\text {max }}^{5 / 2}$ has the prescribed value (4.4). From $n_{0}$, we can deduce

$$
m_{1 / 2}(t=0)=\int_{\mathbb{R}^{+}} S^{1 / 2} n(t=0, S) \mathrm{d} S=\frac{2}{3} n_{0} S_{\text {max }}^{3 / 2} .
$$

From the previous definition for $m_{3 / 2}(0)$, Eq.(4.7) and Eq.(4.5), the reference size of the distribution is computed as $S_{0}=\frac{3}{5} S_{\max }$.

The analytical solution is derived as follows. It is obvious that $K=0$ implies $\partial_{t} m_{1 / 2}=\partial_{t} m_{3 / 2}=0$. Because $\partial_{t} \rho_{g}=0$, the total momentum conservation $\partial_{t}\left(\rho_{g} u_{g}+\frac{1}{6 \pi^{1 / 2}} \rho_{d} m_{3 / 2} u_{d}\right)=0$ can be divided by $\rho_{g}$ and then integrated to yield $u_{g}=-\Phi_{\mathrm{m}} u_{d}+\psi$, with $\psi=0$ thanks to the initial data. Moreover, $\Phi_{\mathrm{m}}$ remains constant in time. A little algebra shows then that

$$
\partial_{t} u_{d}=-\left(1+\Phi_{\mathrm{m}}\right) \frac{18 \pi \mu_{g, 0}}{\rho_{d}} \frac{m_{1 / 2}(0)}{m_{3 / 2}(0)} u_{d}=-\left(1+\Phi_{\mathrm{m}}\right) \frac{1}{\tau_{d}} u_{d}
$$


The solution of (4.8) is the decaying exponential

$$
u_{d}(t)=u_{d}(0) \exp \left(-\frac{t}{\tau_{0}^{b}}\right), \quad \text { with } \tau_{0}^{b}=\frac{\tau_{d}}{\left(1+\Phi_{\mathrm{m}}\right)} .
$$

This represents a two-way coupled velocity relaxation due to the drag between the phases at relatively high mass loading.

At the numerical level, the stability property of SDIRK depends only the ratio $\Delta t / \tau_{0}^{b}$. However, we prefer working with the more convenient ratio $\mathrm{Dt}=\Delta t / \tau_{d}=\Delta t /\left(\tau_{g, 0} \mathrm{St}\right)$, which we call the dimensionless time-step. In this case, one has $S_{0}=4.74405 \cdot 10^{-8}$, that leads to $\tau_{d}=3.204 \cdot 10^{-2}$ and St $=4.005$. Smaller Dt leads to more accurate solutions in time. Simulations are run from $t=0$ to $t=0.5 \mathrm{~s}$. and results are displayed in Fig. 1. The numerical solution is in excellent agreement with the analytical one for a small enough time-step $\Delta t=10^{-3}$ with $\mathrm{Dt}=0.0312$. Even when $\Delta t=10^{-1}$ with $\mathrm{Dt}=3.12$, a stable solution is obtained, in which the velocities are slightly oscillating but relaxing toward the proper equilibrium.
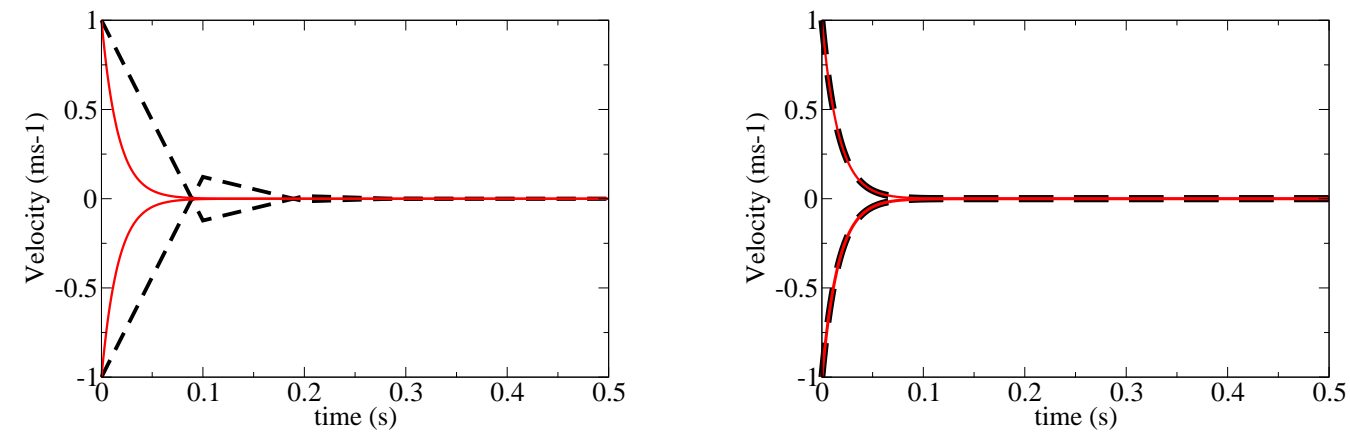

Figure 1: Evolution of spray and gas velocities according to the SDIRK method (black dashed line) and the exact solution (solid red line). The time-step $\Delta t$ is taken to be $10^{-1} \mathrm{~s}$ (left) and $10^{-3} \mathrm{~s}$ (right).

\subsubsection{Drag force and evaporation}

The second numerical experiment, with $K=2 \cdot 10^{-8} \mathrm{~m}^{2} / \mathrm{s}$, is set up to assess the prediction capability of the numerical integrator, as well as the accuracy of the fractional moment reconstructor. The impact of the nonzero value of $K$ can be captured by the characteristic evaporation time

$$
\tau_{v}=\frac{S_{0}}{K}=0.066098 \mathrm{~s}
$$

and its ratio over gas characteristic time

$$
\mathrm{K}_{\mathrm{v}}=\frac{\tau_{v}}{\tau_{g, 0}}=8.26225
$$

For evaporation to have a sensible effect, we consider an exponential distribution in size of droplets

$$
n(t=0, S)=n_{0} \exp \left(-\frac{3}{2} \frac{S}{S_{0}}\right)
$$

in such a way that $S_{0}$ is the mean surface defined by Eq.(4.5). We also define a maximal droplet size in a quite arbitrary way by $S_{\max }=60 S_{0}$ which makes $n\left(t=0, S_{\max }\right)$ almost vanish. We choose $n_{0}$ so that $m_{3 / 2}(t=0)=\int_{\mathbb{R}^{+}} S^{3 / 2} n(t=0, S) \mathrm{d} S=n_{0}\left(2 S_{0} / 3\right)^{5 / 2} \int_{\mathbb{R}^{+}} \varsigma^{3 / 2} \exp (-\varsigma) \mathrm{d} \varsigma$ has the prescribed value (4.4). From $n_{0}$, we can deduce

$$
m_{1 / 2}(t=0)=\int_{\mathbb{R}^{+}} S^{1 / 2} n(t=0, S) \mathrm{d} S=n_{0}\left(\frac{2 S_{0}}{3}\right)^{3 / 2} \int_{\mathbb{R}^{+}} \varsigma^{1 / 2} \exp (-\varsigma) \mathrm{d} \varsigma .
$$


The analytical solution is derived as follows. Starting from $\partial_{t} n-K \partial_{S} n=0$, we have $n(t, S)=$ $n(0, S+K t)$. By virtue of $(4.11), n(t, S)=n(0, S) \exp \left(-\frac{3}{2} K t / S_{0}\right)$. Therefore,

$$
m_{1 / 2}(t)=m_{1 / 2}(0) \exp \left(-\frac{3 K t}{2 S_{0}}\right), \quad m_{3 / 2}(t)=m_{3 / 2}(0) \exp \left(-\frac{3 K t}{2 S_{0}}\right) .
$$

Inserting (4.13) in $\partial_{t} \rho_{g}=\frac{1}{4 \pi^{1 / 2}} \rho_{d} K m_{1 / 2}$ results in

$$
\rho_{g}(t)=\rho_{g}(0)+\frac{\rho_{d} m_{3 / 2}(0)}{6 \pi^{1 / 2}}\left[1-\exp \left(-\frac{3 K t}{2 S_{0}}\right)\right] .
$$

Equations (4.13) and (4.14) confirm the expectation that due to evaporation, the spray mass concentration $\frac{1}{6 \pi^{1 / 2}} \rho_{d} m_{3 / 2}$ should decrease and the gas density $\rho_{g}$ should increase.

The momentum conservation $\partial_{t}\left(\rho_{g} u_{g}+\frac{1}{6 \pi^{1 / 2}} \rho_{d} m_{3 / 2} u_{d}\right)=0$ is integrated to give $\rho_{g} u_{g}+\frac{1}{6 \pi^{1 / 2}} \rho_{d} m_{3 / 2} u_{d}=$ $\Gamma$, with $\Gamma=0$ thanks to the initial data. Thus, $u_{g}=-\phi_{m} u_{d}$ where

$$
\phi_{m}(t):=\frac{\rho_{d}}{6 \pi^{1 / 2}} \frac{m_{3 / 2}(t)}{\rho_{g}(t)}=\frac{\Phi_{\mathrm{m}} \exp \left(-\frac{3 K t}{2 S_{0}}\right)}{1+\Phi_{\mathrm{m}}\left[1-\exp \left(-\frac{3 K t}{2 S_{0}}\right)\right]},
$$

is now a function of time. Note that $\phi_{m}(0)=\Phi_{\mathrm{m}}$ defined in $\S 4.1 .1$. Combining $\partial_{t}\left(m_{3 / 2}\right)=-\frac{3}{2} K m_{1 / 2}$ with $\partial_{t}\left(m_{3 / 2} u_{d}\right)=-\frac{3}{2} K m_{1 / 2} u_{d}-\frac{18 \pi \mu_{g, 0}}{\rho_{d}} m_{1 / 2}\left(u_{g}-u_{d}\right)$ and using (4.15), we end up with

$$
\partial_{t} u=-\left(1+\phi_{m}(t)\right) \frac{18 \pi \mu_{g, 0}}{\rho_{d}} \frac{m_{1 / 2}(t)}{m_{3 / 2}(t)} u_{d}=-\left(1+\phi_{m}(t)\right) \frac{1}{\tau_{d}} u_{d} .
$$

The solution of $(4.16)$ is then

$$
u_{d}(t)=u_{d}(0) \exp \left(-\frac{t}{\tau_{d}}\right)\left[1+\Phi_{m}\left(1-\exp \left(-\frac{3 K t}{2 S_{0}}\right)\right)\right]^{-\frac{2 S_{0}}{3 K \tau_{d}}} .
$$

Equations (4.17) underscore a nonlinear exponential velocity relaxation due to the drag force.

Simulations are run from $t=0$ to $t=0.5 \mathrm{~s}$. The results are displayed in Fig. 2 . The dimensionless timestep still has the same definition $\mathrm{Dt}=\Delta t / \tau_{d}$ as before. According to the considered size distribution, the characteristic spray dynamic time scale and Stokes number for the mean droplet size $S_{0}=1.32196 \cdot 10^{-9}$ are computed as $\tau_{d}=8.92815 \cdot 10^{-4}$ and $\mathrm{St}=0.11160$ respectively. An accurate set of computed results, with relative errors below $1 \%$ for all quantities, is obtained for time step $\Delta t=10^{-3}$ with $\mathrm{Dt}=1.12007$. The solution remains stable even for larger time-steps leading to a very robust numerical scheme with proper prediction of the asymptotic states.

\subsection{Transport of moments in one-way coupling}

To test the method for phase C presented in $\S$ Appendix A.3, we concentrate on 1-D cases, for which a one-way coupling dedicated prototype has been written using the ALE formalism. This prototype works with dimensionless units, and therefore all variables in this subsection will be nondimensional. To alleviate the notations, we shall deliberately omit the $*$-superscript.

\subsubsection{Evaporating aerosol}

The first numerical experiment is aimed at demonstrating the possibility of transporting and evaporating an aerosol, as well as the need for a second-order scheme on a moving grid. The particles are considered as tracers, or passive scalars, for the gas phase. In other words, instead of having a momentum balance giving $\partial_{t}\left(m_{1} u\right)$, we simple-mindedly force $u=u_{g}$. Consequently, the phase $\mathrm{C}$ equations for the aerosol are

$$
\begin{aligned}
& \partial_{t}\left(J_{g}\right)+J_{g} \partial_{x}\left(u_{g}-w\right)=0 \\
& \partial_{t}\left(J_{g} \boldsymbol{m}\right)+J_{g} \partial_{x}\left(\boldsymbol{m}\left(u_{g}-w\right)\right)=0 .
\end{aligned}
$$



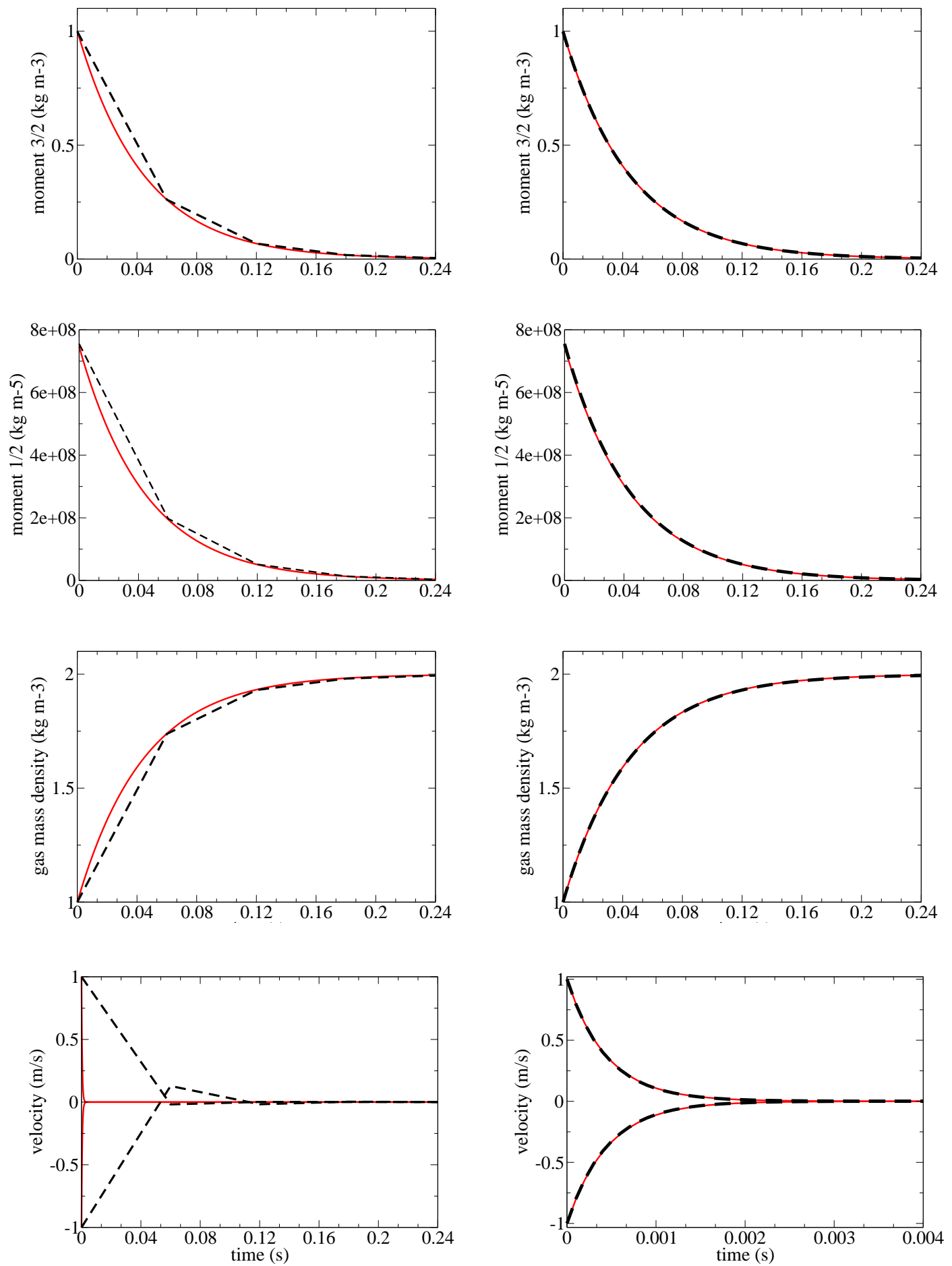

Figure 2: Evolution of various spray and gas properties. Results from analytical (solid red lines) and numerical (dashed black lines) solutions. Cases for $\Delta t=0.06 \mathrm{~s}$ (left column), for $\Delta t=10^{-3} \mathrm{~s}$ (right column). Top row: moment of order $m_{3 / 2}$; second row: moment of order $m_{1 / 2}$; third row: gas mass density $\rho_{g}$; bottom row: velocities $u_{d}$ and $u_{g}$. 
The gas is initiated as the Riemann data

$$
\left(P_{g}, \rho_{g}, u_{g}\right)(t=0, x)=(3,1,1)-\left(2, \frac{7}{8}, 0\right) \mathbf{H}\left(x-\frac{1}{2}\right),
$$

where $\mathbf{H}$ stands for the Heaviside function. The aerosol has an initial NDF equal to

$$
n(t=0, x, S)=\mathbf{1}_{[0,1]}(S)\left[1-\frac{7}{8} \mathbf{H}\left(x-\frac{1}{2}\right)\right] .
$$

The reason why $\rho_{g}(0, x)$ has been set identical to $m_{0}(0, x)=\int_{\mathbb{R}^{+}} n(0, x, S) \mathrm{d} S$ is for their profiles to be easily comparable. The initial data are plotted in Fig. 3-left.

The Neumann boundary conditions are applied at the boundaries of the space interval [0,1], which is discretized by a uniform mesh of 200 cells. Some attention must be paid to the choice of the time-step. Indeed, the time-step is determined at the beginning of the resolution, prior to phase A. Therefore, we can at best control $\left|u_{g}{ }^{n}-w^{n}\right| \Delta t / \Delta x^{n}$. However, during phases A and B, both $u_{g}$ and $\Delta x$ undergo changes, so that the CFL condition (A.27) may be violated in phase C, in terms of $\left|u_{g}{ }^{B}-w^{n}\right| \Delta t / \Delta x^{B}$.

It is wise to start with a non-evaporating simulation in which $K=0$, just to check that waves are correctly propagated. Figure 3-right depicts the analytical and computed solutions in $\left(\rho_{g}, m_{0}\right)$ at $t=0.1$. Of course, $m_{2}$ and $m_{3}$ are also solved, but not shown here. The moment $m_{0}$ even seems to be less dissipated than the density $\rho_{g}$. The first conclusion is that the ALE method gives convincing results as far as transport and acoustic phenomena are concerned.

We are now ready to switch to a genuinely evaporating case with $K=2$. Figure 4-left plots the solution at $t=0.1$. It shows that, in addition to being propagated and contrary to the gas density $\rho_{g}$, the droplet number density $m_{0}$ is also attenuated by a rate of about 2, going from 1 to 0.8 for $x$ small enough, say, $x<0.2$. This observation can be explained by inspecting the right-hand side of the equation $\partial_{t}\left(m_{0}\right)+\partial_{x}\left(m_{0} u_{d}\right)=-K n(S=0)$. Assuming $u_{d}=u_{g}=: u$ remains uniform for $x$ small enough, we have $n(t, x, S)=n(0, x-u t, S+K t)$ from $\partial_{t} n+u \partial_{x} n-K \partial_{S} n=0$. Therefore, $n(t, x, S=0)=n(0, x-u t, K t)$ and according to $(4.20), n(0, x-u t, K t)=1$ as long as $t<1 / K$ and $x-u t<0.5$. Hence, $-K n(S=0)=-2$ for $x$ small enough.

Figure 4-right displays the results for $m_{0}$ of the same test case, but comparing a first-order scheme with the second-order scheme. The accuracy of these schemes is compared on a fixed grid but also in a moving grid. The grid is subject to a uniformly oscillating motion

$$
w(t, x)=4 \pi \cos (20 \pi t),
$$

which creates sinusoidal trajectories

$$
x(t, \chi)=\chi+0.2 \sin (20 \pi t) .
$$

The curved are zoomed in on the interface region. For a fixed grid, the second-order scheme is already more accurate than the first-order. But the interest of a second-order scheme becomes obvious when the grid moves. In our case, the high grid velocity leads to small CFL numbers for the fluids. Therefore the profile of $m_{0}$ is much more diffused with the first-order scheme than with the second-order scheme.

\subsubsection{Non-evaporating spray}

The second numerical experiment is aimed at demonstrating the stability as well as the good level of accuracy of the transport scheme in a staggered grid for a non-evaporating spray. In particular, we want to show that the convection solver is general and does not presume any type of NDF. For that purpose, we consider the NDF

$$
n(t=0, x, S)=\left[(1-2 x)^{2} \sin (\pi S)+4 x(1-x) \exp (-10 S)\right] \mathbf{1}_{[0,1]}(S) \mathbf{1}_{[0,0.5]}(x),
$$

which is a convex combination of $\sin (\pi S)$ and $\exp (-10 S)$ for $x \in[0,0.5]$. The moments associated to this NDF are subject to a discontinuous initial velocity

$$
u_{d}(t=0, x)=0.5+1.5 \mathbf{H}(x-0.25) .
$$

Periodic boundary conditions are applied at the boundaries of the space interval $[0,1]$, which is discretized by a uniform mesh of 200 cells. The analytical solution is the translation of the two parts of 

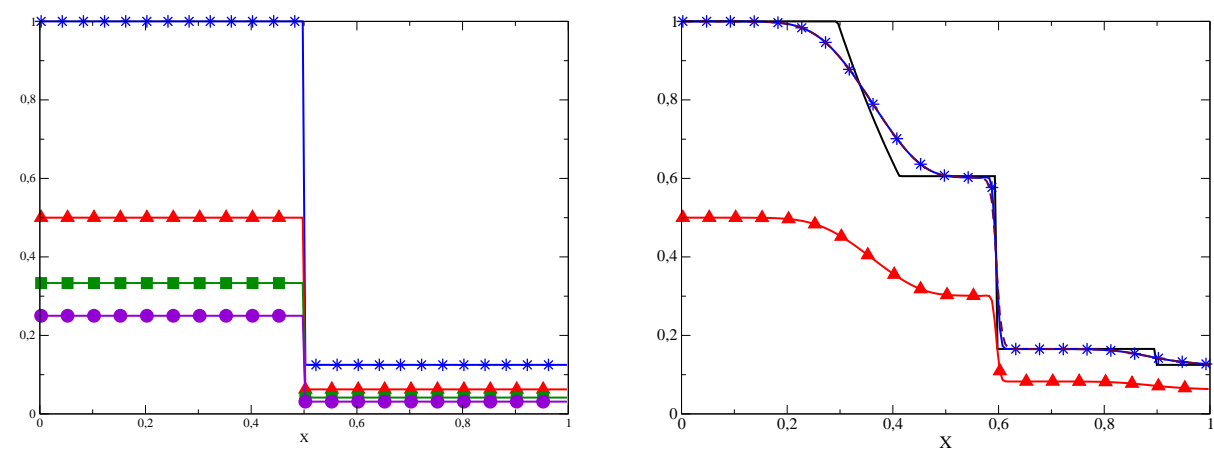

Figure 3: Left: Initial data for the moments $m_{0}$ (blue line with stars), $m_{1}$ (red line with triangles), $m_{2}$ (green line with squares), $m_{3}$ (purple line with circles). Right: Solution of the Riemann problem at $t=0.1$ for non-evaporating particles.
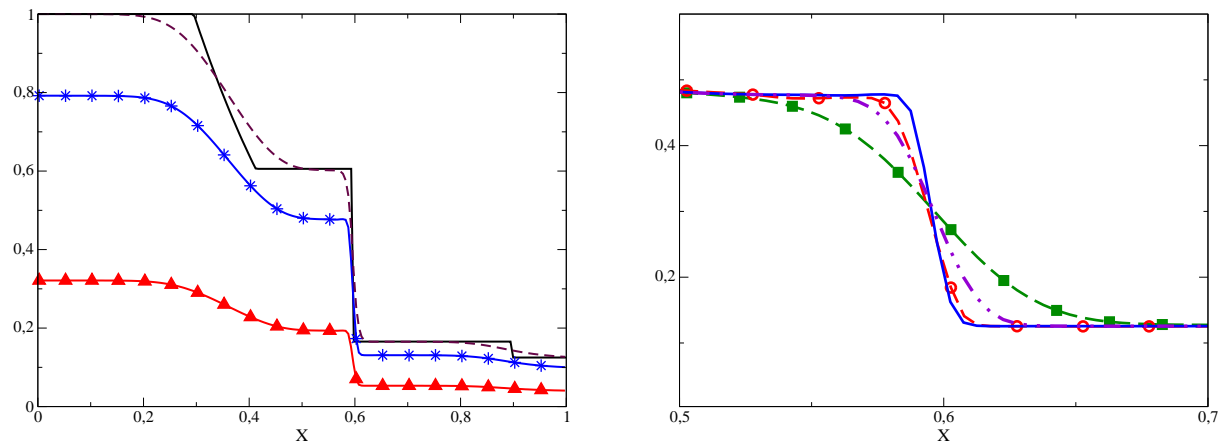

Figure 4: Left: Solution of the Riemann problem at $t=0.1$ for evaporating particles, containing the exact $\rho_{g}$ (solid black line), the computed $\rho_{g}$ (dashed brown line), the computed $m_{0}$ (blue curve with circles) and $m_{1}$ (red curve with triangles). Right: Close-up on the interfacial area and comparison between various solutions for $m_{0}$, namely, second-order solution without mesh movement (solid blue line), second-order solution with mesh movement (dashed red line), first-order solution without mesh movement (dotted-dashed purple line), first-order solution with mesh movement (green line with squares).
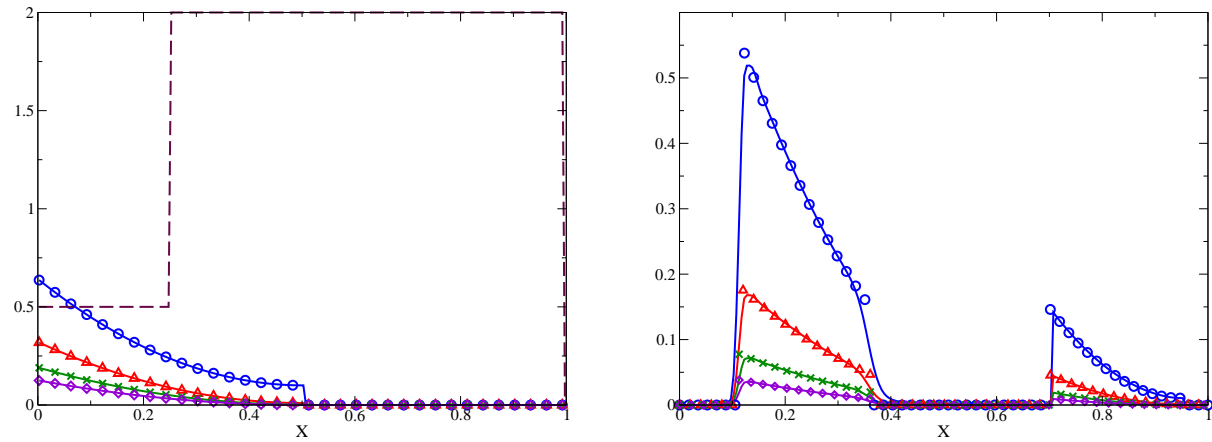

Figure 5: Evolution of a spray in a discontinuous velocity field. Left: Initial data for the moments and the velocity. Right: Analytical solutions (markers) and computed solutions (lines) at time $t=0.225$ in the first four moments, i.e., $m_{0}$ (blue), $m_{1}$ (red), $m_{2}$ (green), $m_{3}$ (purple).

the density profile corresponding to each value of the velocity. Figure 5-left displays the initial condition for the size moments and the velocity.

At $t=0.225$, the initial distribution breaks into two parts, as shown in Fig. 5-right. Vacuum is created at the prescribed velocity discontinuity. The computed moments are represented by solid lines in decreasing order, meaning that the highest curve stands for the 0th-order moment, and the lowest 
curve stands for the 3rd-order moment. Their analytical counterparts are represented by markers. The numerical solution is seen to match the analytical one very accurately. We underline that the proposed model and numerical method with built-in realizability condition have provided a robust way of treating the coupled dynamics and evaporation of a polydisperse spray carried by a gaseous flow field, even within the moving mesh framework, the appearance of vacuum zones of droplets and with potentially very stiff source terms treated implicitly.

\section{Implementation of EMSM in IFP-C3D}

\subsection{A glimpse of IFP-C3D}

Over the past several years, the IFP-C3D code [5] has been developed at IFP Energies nouvelles for the numerical simulation of advanced internal combustion engines. IFP-C3D involves a great many intricate physical and chemical processes, most prominently transient and multidimensional, for multicomponent mixtures of gases undergoing mixing, ignition, reactions, heat transfer, and turbulence. These numerous options can be individually activated or deactivated by input switches, which makes IFP-C3D a versatile tool for engineers. As far as liquid sprays are concerned, it was previously incorporated in IFP-C3D with a two-way coupled Lagrangian approach called stochastic parcel [32].

The most salient feature of IFP-C3D is its capability to tackle engine geometries with arbitrarily shaped moving piston, valves or other actuators. To accomplish this, IFP-C3D resorts to a staggered grid whose hexahedral or tetrahedral cells build up an unstructured mesh. Thermodynamic variables are located at the cell-centers, while velocity vectors are located at the nodes. Such a choice is meant to make the mesh motion unambiguous: the vertices are simply moved to new user-specified positions. The price to be paid for such a convenience with respect to mesh motion is some awkwardness in discretizing the momentum balance equations over the dual mesh.

As expounded in $\S 3.2$ and $\S 3.3$, each time-step in IFP-C3D consists of three phases:

- In phase A, source terms of the chemical reactions on gas ${ }^{9}$, of the Lagrangian fuel injection spray and of the spark ignition are calculated.

- In phase B, all the diffusive and acoustic terms are solved implicitly, with first the species mass, internal energy term, and turbulent terms. The method introduced in [53] is retained in its fully implicit version. The coupled implicit equations (momentum, temperature and pressure) are solved with the SIMPLE algorithm [61]: this is called the PVT (Pressure Temperature Velocity) loop.

- In phase $\mathrm{C}$, the outcomes of phase B are remapped in order to match the imposed new grid. The corresponding linear convection equations are solved explicitly, so as to enhance accuracy with respect to kinematic phenomena. Limited slope reconstruction is considered via dimensional splitting, which gives rise to the so-called Quasi Second Order Upwind Scheme (QSOU) [2]. Subcycles may have to be introduced in order to ensure the CFL condition.

\subsection{Verification and Validation in one-way coupling configurations}

In light of the numerical method outlined in $\S 3$ and $\S$ Appendix $A$, we have added to IFP-C3D the ability to simulate a Eulerian spray using the EMSM model. An account of this work can be found in [22]. This implementation is assessed through a range of test cases, the two most crucial of which are presented below in the one-way coupling configuration. We propose to first verify this implementation in the context of mesh movement and creation of singularities in a $1 \mathrm{D}$ case. We then move to a $2 \mathrm{D}$ case where we still verify the robustness of the method in critical cases where delta-shocks occur. In this $2 \mathrm{D}$ vortex case, we also come-up with a validation of the method, through a comparison with Muses3D, in the context of the polydisperse spray transport and drag. In $\S 6$, an injection case of a polydisperse droplet spray will be done in a two-way coupling framework integrated in the ALE algorithm and compared with the Lagrangian result.

\footnotetext{
${ }^{9}$ auto-ignition, combustion, post-oxidation, chemical equilibrium, etc.
} 


\subsubsection{Scheme robustness through mesh movement}

The objective of this test case is to ensure that the implemented model is stable with mesh movement. The evolution of homogeneous fields of liquid and gas are considered in a closed high pressure cell. The bottom boundary of this cell corresponds to a moving piston being at the bottom dead center. The gas is taken as air, and the particles are initially stationary. No ignition occurs, and no thermal effect is considered. Also, no special treatment of the boundary is considered. The computation ends after a revolution of the crank, with the crank angle degree (cad) ranging in $\left[-180^{\circ}, 180^{\circ}\right]$. The high pressure cell and the movement of the piston are described in Fig. 6.
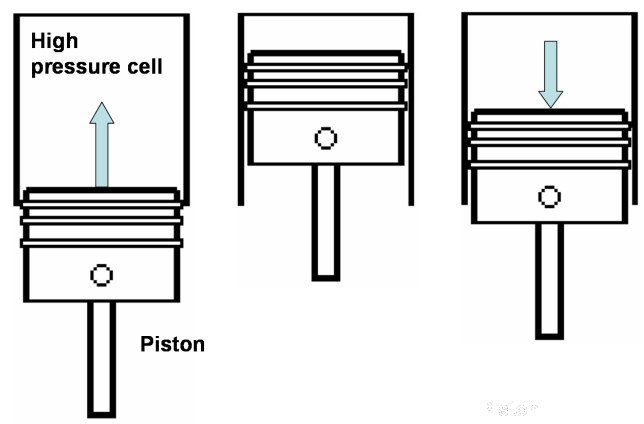

Figure 6: Piston movement during the computation. The computation starts at $\mathrm{cad}=-180^{\circ}$ and ends at $\operatorname{cad}=180^{\circ}$.

The boundary conditions are $\left(\boldsymbol{u}_{\boldsymbol{d}}-\boldsymbol{w}\right) \cdot \boldsymbol{n}=0$ on the surface of the piston, and $\boldsymbol{u}_{\boldsymbol{d}} \cdot \boldsymbol{n}=0$ at the upper edge of the domain. The size distribution is constant and similar to the one-dimensional tests with an aerosol. During the compression and expansion of the high pressure cell, the computational cell volumes change is homothetic. The results are displayed for the number density $m_{0}$ and the surface density $m_{1}$ with a 1200 -cell grid $(30 \times 40)$. Computations are successively run for an aerosol and a spray. Figure 7-left displays the results for the moments, in the case of an aerosol, at cad $=-100^{\circ},-30^{\circ}, 50^{\circ}, 180^{\circ}$. In the various graphs, the distance where the value of the moments is null is the distance traveled by the piston. These graphs show that the flow stays homogeneous during the whole computation. This is a typical case where the operator splitting done in the algorithm of IFP-C3D is legitimate since the speed of sound is at least one order of magnitude higher than the convective speed of the fluid. In fact, the speed of sound exceeds $300 \mathrm{~m} / \mathrm{s}$. At the same time, with a rating of $1200 \mathrm{rpm}$, and a stroke of about $10 \mathrm{~cm}$, the piston average velocity and that of the dragged fluid is much smaller than the speed of sound in the gas. Mass conservation imposes that the gas density and consequently the particle number increases as the piston heads to the top dead center, because the volume of the high pressure cell decreases, Fig. 7-left (top right), Fig. 7-left (bottom left). The moments recover their initial values at the end of the computation which corresponds to the end of the expansion stroke.

Figure 7-right displays results for a spray at different instants. The first two $\left(\mathrm{cad}=-100^{\circ}\right.$ and $\left.\mathrm{cad}=-30^{\circ}\right)$ correspond to the compression of the high pressure cell. The two following ones $\left(\mathrm{cad}=50^{\circ}\right.$ and cad $=180^{\circ}$ ) correspond to the expansion of the domain. The case of a spray is dramatically different from the previous one. Indeed inertial particles within the infinite Stokes limit are considered now, since no interaction with the gas phase is taken into account. They stick to the piston as it moves upward. This behavior is observed in Fig. 7-right (top) and is responsible for the singularity present at the piston surface. Meanwhile, the moment field downstream of the piston is unchanged. This is a direct consequence of the assumption made for pressureless gas dynamics, wherein the speed of sound is null, and consequently no pressure wave modifies the field. In Fig. 7-right (bottom), the $\delta$-shock moves forward relatively to the mesh. The enlargement of the $\delta$-shock has two explanations. The first one is the numerical diffusion. Secondly, the computational cell volume expansion contributes to the $\delta$-shock enlargement. Meanwhile, upstream of the $\delta$-shock, in the wake of the piston, a vacuum zone is created, which is the expected behavior. 

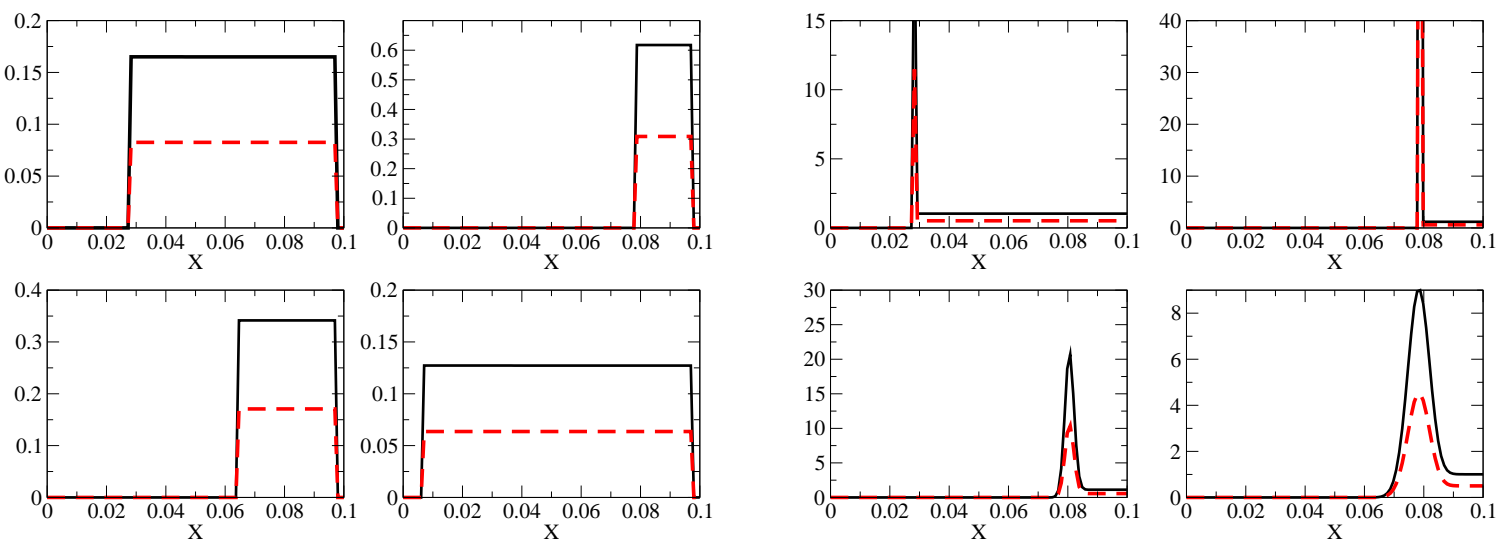

Figure 7: Left: Results in the case of an aerosol, for $m_{0}$ (solid black line) and $m_{1}$ (dashed red line). Results for cad $=$ $-100^{\circ},-30^{\circ}, 50^{\circ}, 180^{\circ}$. Right: Results in the case of a spray, for $m_{0}$ (solid black line) and $m_{1}$ (dashed red line). Results for $\mathrm{cad}=-100^{\circ},-30^{\circ}, 50^{\circ}, 180^{\circ}$.

\subsubsection{Spray dynamics through evaporation and drag, comparison with MUSES3D}

The objective of this test case is to study and validate the behavior of spray particles, undergoing evaporation and drag. Therefore no mesh movement is considered. The chosen test case involves the Taylor-Green vortices for the gas velocity field in a square and periodic domain, wherein a motionless droplet cloud is initiated, which can be seen in Fig. 8. The results obtained with IFP-C3D are validated by comparison with the results obtained for the same configuration with the MUSES3D code, an academic solver for polydisperse spray [9], wherein the EMSM model has been implemented and validated [39].

The velocity field then writes

$$
\boldsymbol{u}_{\boldsymbol{g}}(x, y)=U_{0}\left(-\sin \frac{2 \pi x}{L_{0}} \cos \frac{2 \pi y}{L_{0}}, \cos \frac{2 \pi x}{L_{0}} \sin \frac{2 \pi y}{L_{0}}\right) .
$$

The characteristic droplet diameter is taken as $100 \mu \mathrm{m}$ which amounts to a Stokes number of 1.4

Four different instants are represented. The results obtained with the IFP-C3D code are compared to the ones obtained with the MUSES3D code at $t=1$, Fig. $9, t=1.4$, Fig. 10 , and $t=2$, Fig. 11 . The field displayed is $m_{0}$, size moment of order zero, equal to the total droplet number.

Since the droplet cloud is initialized in two different vortices, a main part is dragged in the top-left vortex, whereas a small part is dragged by the top-right vortex, as seen at $t=1$. The droplet Stokes number is much higher than the critical Stokes number $\mathrm{St}_{c}$, below which the particles stay in the vortices, so that their characteristics do not cross. At $t=1.4$, particles are ejected from the vortices because of the periodic boundary conditions, especially particles dragged by the top-left vortex which enter the bottom-right vortex. In this region, the gas velocity field direction is opposed to the droplet velocity one. As seen at $t=2$, this leads to the creation of a discontinuity in the velocity and thus to the formation of a $\delta$-shock in the particle size moment field. We reiterate that this is a typical effect of the pressureless gas formalism used in the EMSM model. Meanwhile, the residual portion of the small part dragged by the top-right vortex is ejected from this vortex and enters the $\delta$-shock.

Although the code structures are very different, one can assess that the level of similarity between the results given by the two codes is very high. Indeed there are three paramount differences between the numerical schemes implemented in MUSES3D and in IFP-C3D. The first one concerns the formalism used. The MUSES3D code is an academic solver dedicated to spray resolution in an Eulerian formalism, whereas in IFP-C3D the ALE formalism is implemented. Secondly, the numerical scheme for transport in physical space is second order in space and time in the MUSES3D code, whereas it is only first order in the IFP-C3D code as indicated in Table 1. This is the reason why the moment field is more diffused at time $t=1,1.4$ and 2. Finally, in the structured MUSES3D code, a dimensional splitting algorithm handles transport in multi-dimensions, whereas in the IFP-C3D code which is an unstructured 
code, it is fully multi-dimensional. This is why, at time $t=2$, in the result given with MUSES3D, the particles, dragged by their inertia, penetrate further in the bottom-right vortex than in the result given in the IFP-C3D code. Nevertheless, this comparison case is a success and allows us to verify the model implementation in the IFP-C3D code, coming up with a robust singularity handling moment-space preserving ALE numerical method. Furthermore, in the context where no singularity occurs, it validates the new IFP-C3D polydisperse spray model, in terms of spray dynamics through transport coupled to drag and evaporation.
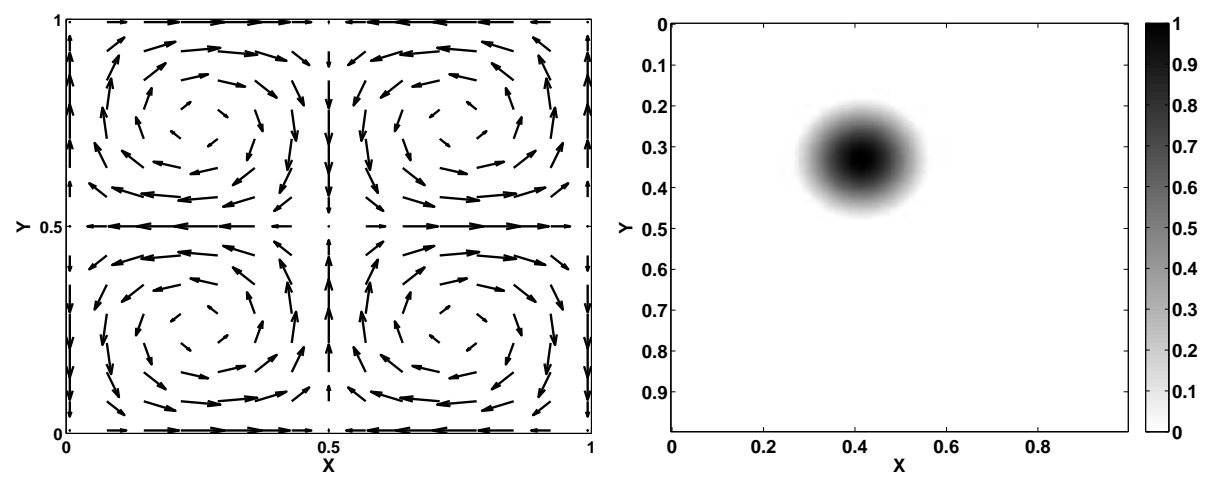

Figure 8: Left: Taylor-Green configuration for the gaseous flow. Right: initial condition for the moment $m_{0}$ of the spray.
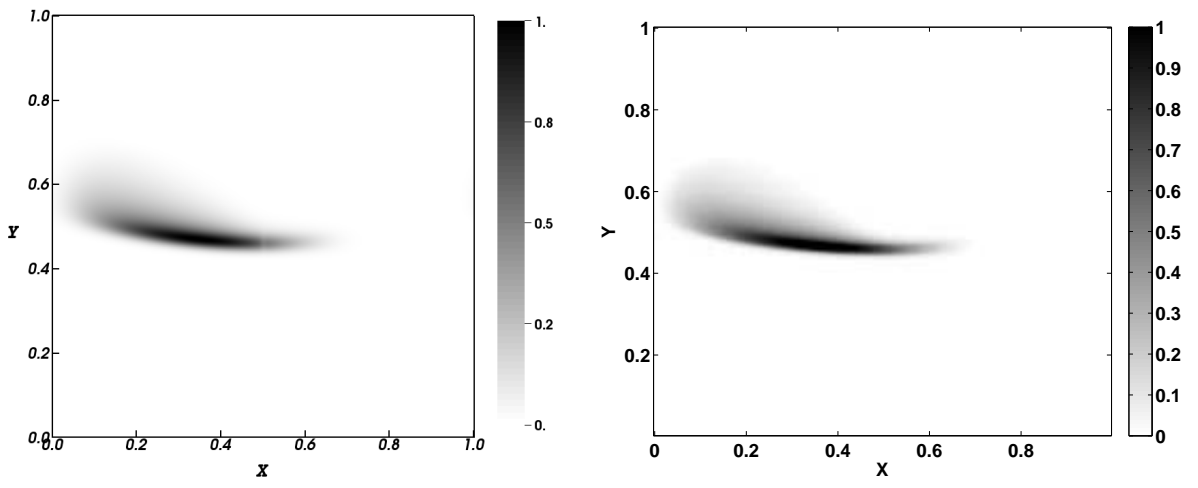

Figure 9: Results for $m_{0}$ at time $t=1$. Left: with IFP-C3D. Right: with MUSES3D.

\begin{tabular}{lccc}
\hline \hline & MUSES3D & ALE research code & IFP-C3D \\
\hline moment vector & $2^{\text {nd }}$ order & $2^{\text {nd }}$ order $/ 1^{\text {st }}$ order & $1^{\text {st }}$ order \\
velocity vector & $2^{\text {nd }}$ order & $1^{\text {st }}$ order & $1^{\text {st }}$ order \\
mesh motion & No & Yes & Yes \\
\hline
\end{tabular}

Table 1: Order of the numerical schemes used for the resolution of the transport in physical space. ALE research code was used to obtain the results presented in subsection 4.2 while MUSES3D and IFP-C3D were used to produce the results presented in subsection 5.2.

\section{Feasibility of injection computations with IFP-C3D}

The $2 \mathrm{D}$ test-case involved in section 5 was dedicated to an academic framework where the gas-phase is considered to be frozen and the cloud of polydisperse droplets initially at zero velocity is driven by the 

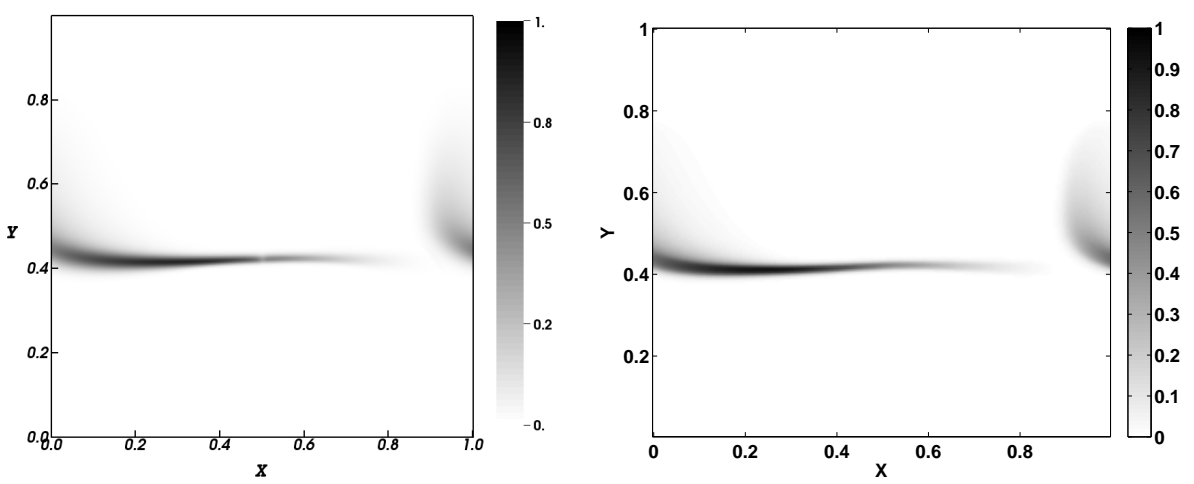

Figure 10: Results for $m_{0}$ at time $t=1.4$. Left: with IFP-C3D. Right: with MUSES3D.
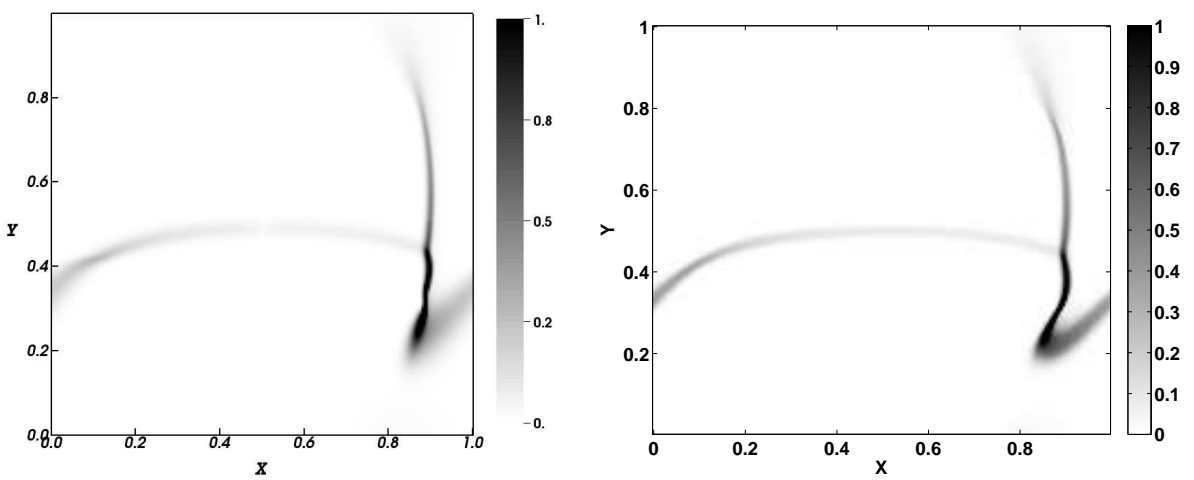

Figure 11: Results for $m_{0}$ at time $t=2$. Left: with IFP-C3D. Right: with MUSES3D.

gas. However, this study is not enough to prove the ability of moment methods to reproduce correct and stable simulations for a more complicated application. Moreover, in the context of industrial applications such as the direct injection of the liquid fuel in an internal combustion engine, it is required to capture the gas-phase entrainment by the polydisperse spray. Therefore, the efficiency of the two-way coupling methodology developed in subsection 3.1 needs to be investigated under the injection computations with IFP-C3D. Let us recall that injection parameters for the spray should be chosen such that the mass loading ratio $\Phi_{m}$ and the spray volume fraction $\Phi_{v}$ belong to a range where the two-way coupling is valid, as highlighted by in [4]. Yet, the issue of spatial discretization accounts for a difficult task. The finite volume method on staggered grids [43] along with the mesh motion through ALE formalism can be considered as major numerical difficulties within the framework of engine simulations, tackled in the industrial software IFP-C3D [5]. Moreover, the moment space conservation issue through EMSM still needs to be approved. In this section, one considers a 2-D injection of the gas-spray mixture in a gas filled chamber. Actually, the test case is chosen such that one has the corresponding experimental data for a thin, polydisperse and collisionless spray under the turbulent dispersion of droplets [25]. However, we will limit our study to a laminar flow regime. The industrial software IFP-C3D [5] dedicated to reactive compressible flows will be used for such simulations. In the following, the test case, including both spray and gas properties, will be first described. The main aim being to verify the full Eulerian description of the dispersed phase with respect to Lagrangian particles [21], one will then introduce the methodology to correctly initialize both Eulerian and Lagrangian fields at the beginning of each computation. Finally, injection results will be discussed. The effect of mean droplet size with and without evaporation will be investigated. 


\subsection{Description of the test case}

The computations are run with a polydisperse droplet population governed by the Rosin-Rammler distribution [56]

$$
f(r)=q \frac{r^{q-1}}{r_{\mathrm{SMR}}^{q}} \exp \left(-\frac{r^{q}}{r_{\mathrm{SMR}}^{q}}\right),
$$

where $r$ is the particle radius, $r_{\mathrm{SMR}}$ is the Sauter mean radius (SMR), and $q$ is a coefficient determining the sharpness of the distribution. The distribution considered here is the one with $q=3.5$, see Fig. 12 .

In case where no moving parts are considered, the computations are run with the geometry presented in Fig. 13. This is proportional to a two-dimensional mesh in the $y$ - and $x$-axes, respectively. This $2-\mathrm{D}$ plan is composed of square cells with a length of $0.25 \mathrm{~mm}$ (320 cells in the $x$-direction, and 800 cells in the $y$-direction). Yet two cells with a length of $0.5 \mathrm{~mm}$ along the $z$-axis had to be kept since IFP-C3D software is a 3 -D code. The $32 \times 16$ cell additional part at the top of the mesh is dedicated to the injection type inlet condition. Periodic boundary conditions are set on faces orthogonal to the $z$-axis, and free exit boundary conditions are set for the part localized at $y=0, x=40 \mathrm{~mm}$ and $x=-40 \mathrm{~mm}$.

Within the framework of a piston motion, the computations are run with the same geometry as presented in Fig. 13, but with mesh movement. That is, instead of free exit boundary conditions, one considered wall conditions for the parts localized at $x=40 \mathrm{~mm}$ and $x=-40 \mathrm{~mm}$ and moving piston for the bottom boundary part at $y=0$. Let us give the law related to the instantaneous piston velocity $U_{\text {piston }}(t)$ as:

$$
U_{\text {piston }}(t)=U_{m, \text { piston }} \frac{\pi}{2} \sin \left(\theta_{\text {cad }}\right)\left(1+\frac{\cos \left(\theta_{c a d}\right)}{\left[\left(\frac{2 l}{S_{l}}\right)^{2}-\sin ^{2}\left(\theta_{c a d}\right)\right]^{1 / 2}}\right),
$$

with $\theta_{\text {cad }}$ the crank angle degree, $l$ the rod lenght, $S_{l}$ the piston stroke length. Let us recall that the mean piston speed $U_{m, \text { piston }}=2 S_{l} N_{\text {rot }} / 60$ is in function of the the rotational speed in revolutions per minute of the crankshaft $N_{\text {rot }}$.

The cases presented in this section involve injection of a fuel spray and gas with velocity $u_{\text {inj }}=18 \mathrm{~m} / \mathrm{s}$. The ambient gas phase is taken as air, with initial pressure $P_{g}=10^{5} \mathrm{~Pa}$, temperature $T_{g}=293 \mathrm{~K}$, and viscosity $\mu_{g}=1.9910^{-5} \mathrm{~kg} / \mathrm{m} / \mathrm{s}$, whereas the fuel is taken as the $\mathrm{C}_{14} \mathrm{H}_{30}$ species, with a density $\rho_{d}=763 \mathrm{~kg} / \mathrm{m}^{3}$. Some other initial thermodynamic quantities can be found in Table 2 .

\begin{tabular}{l|c}
$C_{p, g}$ & $1014.04730245777 \mathrm{~J} / \mathrm{kg} / \mathrm{K}$ \\
\hline$C_{v, g}$ & $724.659744436102 \mathrm{~J} / \mathrm{kg} / \mathrm{K}$ \\
\hline$\gamma_{g}$ & 1.39934267115507 \\
\hline$e_{g}$ & $21318.245276214 \mathrm{~J} / \mathrm{kg}$ \\
\hline$R_{g}$ & 8.31440 \\
\hline$C_{v, d}$ & $1435.90874724034 \mathrm{~J} / \mathrm{kg} / \mathrm{K}$ \\
\hline$e_{d}$ & $-59784.8813976229 \mathrm{~J} / \mathrm{kg}$
\end{tabular}

Table 2: Values of heat capacity in constant pressure of the gas $C_{p, g}$, in constant volume of the gas $C_{v, g}$ and the fuel spray $C_{v, d}$. Specific internal energies of the gas $e_{g}$ and the spray $e_{d}$, the perfect gas constant $R_{g}$ and the ratio $\gamma_{g}=C_{p, g} / C_{v, g}$.

To characterize the spray/gas interaction regime, we introduce a characteristic length $L_{0}=8 \cdot 10^{-3} \mathrm{~mm}$ which is the injection diameter and a characteristic corresponding time $\tau_{g, 0}=L_{0} / u_{\text {inj }}$ for the gas flow. In our case, the mean droplet diameter being significant for the study of the disperse phase, the characteristic dynamic time scale for the droplet population is given as a function of the SMR number

$$
\tau_{0}=\frac{2 \rho_{d} r_{\mathrm{SMR}}^{2}}{9 \mu_{g, 0}} .
$$

We also define some dimensionless numbers such as the fractional volume occupied by the dispersed phase $\Phi_{\mathrm{v}}$ and the Stokes number St expressed as the ratio of the droplet dynamic time response $\tau_{0}$ over the gas characteristic time $\tau_{g, 0}$. 


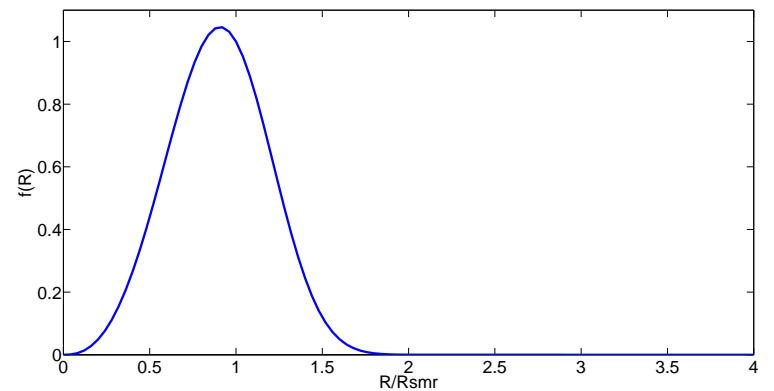

Figure 12: Illustration of Rosin-Rammler distributions actually used in the computations.

\subsection{Initialization strategy of both Eulerian and Lagrangian computations}

Since our aim is to conduct accurate comparisons between Lagrangian and Eulerian simulations, some preliminary computations are required to determine injection boundary conditions. Given the value of injected liquid volume fraction $\alpha_{d}$ and the distribution function shown in Fig. 12, one can compute moments of the distribution at injection for Eulerian simulations. However, in IFP-C3D, the injection of Lagrangian particles is controlled by the total liquid mass $m_{l}$, the injection time length $t_{\text {inj }}$ and the injector radius $r_{\text {inj. }}$. To have the same injection conditions in Lagrangian cases, we first compute the mass flow rate for a given inlet velocity and inlet volume fraction by the formula

$$
\dot{m}_{l}=\Phi_{\mathrm{v}} \rho_{d} u_{\mathrm{inj}} S
$$

where $S$ is the injection section for the two-phase flow and $\rho_{d} S$ corresponds to the injection section for the Lagrangian particles. From the latter, $r_{i n j}$ can be easily calculated. Moreover, from the knowledge of $t_{i n j}, m_{l}$ is deduced from

$$
m_{l}=\dot{m}_{l} t_{\mathrm{inj}} .
$$

Lagrangian particles are injected in the middle of each of the 64 cell face belonging to the inlet condition, which leads to a total of 64 injectors. Since the mesh is a 2-D plane, the radius and the injection section related to each injector remain same.

\begin{tabular}{c|cc}
$r_{\mathrm{SMR}}$ & $5 \mu \mathrm{m}$ & $20 \mu \mathrm{m}$ \\
\hline $\mathrm{St}$ & 0.479 & 7.67 \\
\hline$\Phi_{\mathrm{v}}$ & $2.51 \cdot 10^{-4}$ & $2.51 \cdot 10^{-4}$ \\
\hline$\Phi_{\mathrm{m}}$ & 0.191 & 0.191 \\
\hline$\tau_{v}$ & $1.4 \cdot 10^{-2} \mathrm{~s}$ & $1.4 \cdot 10^{-2} \mathrm{~s}$ \\
\hline$\tau_{v} / \tau_{0}$ & 65.7 & 4.14
\end{tabular}

Table 3: Dimensionless characteristic values at injection: Stokes numbers St, spray volume fraction $\Phi_{\mathrm{v}}$ and mass loading $\Phi_{\mathrm{m}}$ are computed through the Sauter mean radius $r_{\mathrm{SMR}}$.

\subsection{Discussion on injection simulations}

In the context of injection simulations, the two-way coupling effects on flow dynamics can not be neglected when the mass ratio of the dispersed phase over the gas phase becomes significant. Moreover, inertial droplets inside a distribution have a low response to changes in the gas whereas light particles barely follows the carrier gas phase. The spray dispersion is thus influenced by the size of the droplets. In internal combustion engines, a broad range of fuel droplet sizes needs to be considered. We run injection simulations under two types of droplet distributions characterized by different Sauter mean radius $r_{\mathrm{SMR}}$ but the same mass loading $\Phi_{\mathrm{m}}$ or volume fraction $\Phi_{\mathrm{v}}$, as observed in Table 3 . These initial characteristic numbers are chosen such that droplet-droplet interactions can be assumed to be neglected. Note that, we adopt a simple $d^{2}$ evaporation law of droplets under a constant evaporation time $\tau_{v}$ for both low and high 


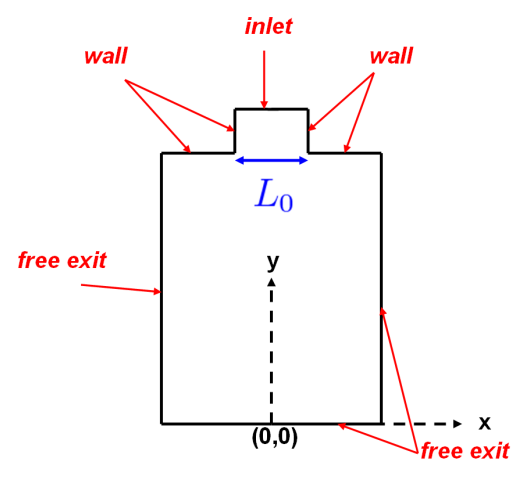

Figure 13: Illustration of the 2-D plane geometry and boundary conditions

inertia droplets. The ratio of the evaporation time over the characteristic droplet time $\tau_{v} / \tau_{0}$, observed in Table 3, shows that the spray with high inertia droplets undergoes a faster evaporation.

For each distribution, we run separately four simulations. Two of them are dedicated to Lagrangian description of droplets whereas the other two are devoted to the full Eulerian resolution of the dispersed flow. Note that for each method, there are two possibilities; the spray being either evaporating or not. The initial spray mass flow rate is taken as $2.76 \cdot 10^{-5} \mathrm{~kg} / \mathrm{s}$. A total number of 6.4 millions numerical particles, insuring the convergence of the Lagrangian solution, is injected in the domain.

In the following, results are displayed for a simulation time of $1.4 \cdot 10^{-2} \mathrm{~s}$. The latter also corresponds to the characteristic evaporation time $\tau_{v}$, as shown in Table 3. Each figure contains results from both Eulerian and Lagrangian resolutions of the fuel spray.

\subsection{Injection of low inertia droplets}

Injection results in the case of a low inertia droplet population with Sauter mean radius $r_{\mathrm{SMR}}=5 \mu \mathrm{m}$ are illustrated in Figures 14 and 15. The fields of spray volume fraction and spray velocity for both Lagrangian and Eulerian sprays are quite similar. The global droplet dynamics are well reproduced. Let us note that the front of the Lagrangian spray is sharper than the Eulerian one.

The fuel vapor field is also very well represented, as seen through Fig. 15. This shows that the mass density modification in the gas due to the evaporation between phases is well captured through the twoway coupling algorithm. Moreover, the gas velocity field driven by the Eulerian spray is qualitatively similar to the one driven by the Lagrangian spray, see Fig. 14. Although the spray and the vapor penetration lengths are slightly different as shown in Fig. 17, there is a good qualitative agreement between both approaches. The spray and vapor dispersions along the injection width are also very well captured despite small differences, as plotted in Fig. 16.

Let us highlight that these small differences between the Lagrangian and Eulerian methods have two origins. First, the level of modeling assumptions, in terms of velocity conditioned on droplet size, is different between the two simulations as already explained in the modeling section. Second, it also partially relies in the difference of spray descriptions. That is, the Eulerian method is derived from the Williams Boltzmann Equation at the kinetic level, which represents an ensemble averaging of an infinite number of flow realizations [67]. On the contrary, the Lagrangian approach, which consists of tracking each individual numerical particle within the context of the Stochastic parcel (SP) modeling, is based on a single flow realization [21]. Moreover, the injection of Eulerian and Lagrangian sprays are not exactly carried out in a similar way in IFP-C3D. At the inlet boundary condition, the spatial distribution of the Lagrangian spray consists in a sum of Dirac delta functions at fixed space locations, whereas the Eulerian spray is homogeneously distributed. In fact IFP-C3D has some limitations in terms of Lagrangian capabilities inherited from previous developments. In this context the Lagrangian simulations offer a reference solution in order to validate the proposed new Eulerian strategy, but a fully quantitative validation and evaluation of the convergence of the Eulerian method as compared to the 

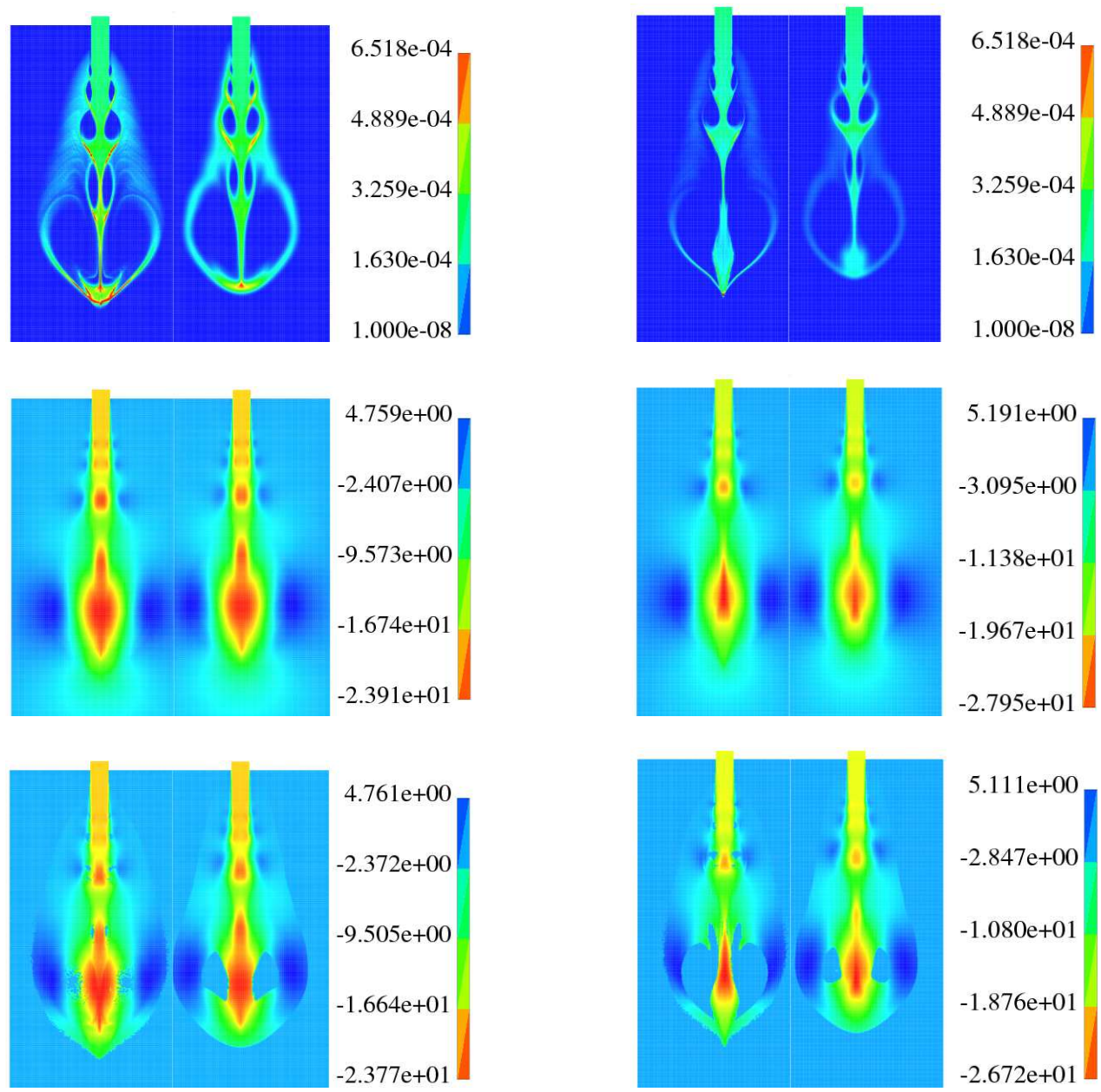

Figure 14: Results for a droplet population of $r_{\mathrm{SMR}}=5 \mu \mathrm{m}$ at time $1.4 \cdot 10^{-2} \mathrm{~s}$. Left: Spray without evaporation. Right: Spray with constant evaporation velocity. Spray volume fraction (top row), gas velocity along the $y$-axis (middle row), spray velocity along the $y$-axis (bottom row). In each panel, Lagrangian spray is displayed on the left side whereas Eulerian on the right side.
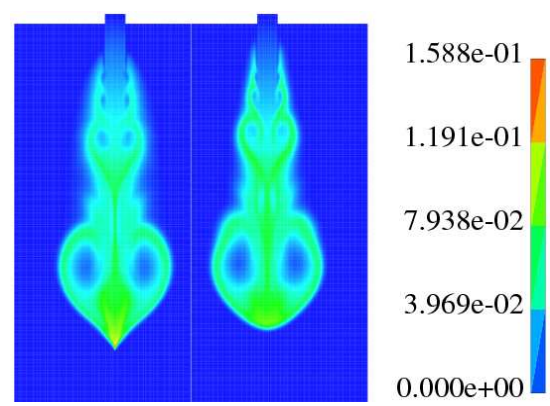

Figure 15: Evaporated fuel mass fraction inside the gas-phase for a droplet population of $r_{\mathrm{SMR}}=5 \mu \mathrm{m}$ under a constant evaporation velocity at time $1.4 \cdot 10^{-2} \mathrm{~s}$. In the panel, Lagrangian spray is displayed on the left side whereas Eulerian is displayed on the right side.

Lagrangian one on this 2D injection two-way coupled configuration is beyond the scope of the present work. 

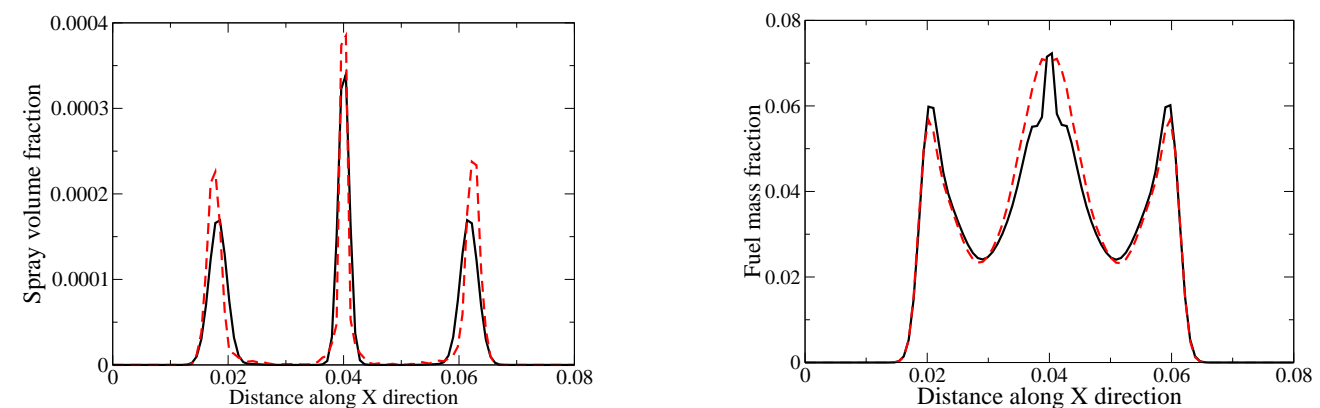

Figure 16: Results from 1-D plots, taken along the $x$-direction which center point on $y$-axis is 12 diameter far from the injector, in case of a droplet population of $r_{\mathrm{SMR}}=5 \mu \mathrm{m}$. Results from Eulerian (solid black lines) and Lagrangian (dashed red lines) solutions. Left: Spray volume fraction for droplets without evaporation. Right: Evaporated fuel mass fraction for evaporating droplets with a constant evaporation velocity.
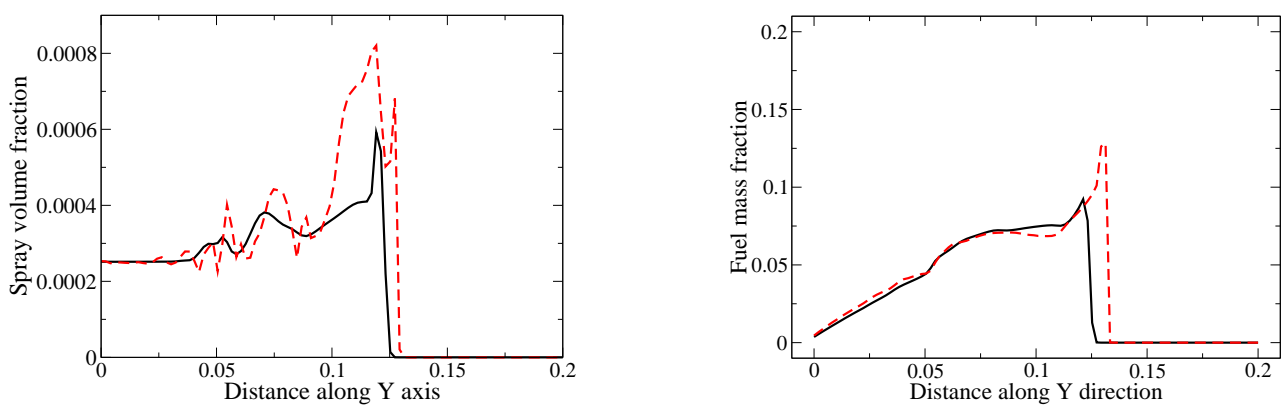

Figure 17: Results from 1-D plots, taken along the $y$-axis see Figure 13, in case of a droplet population of $r_{\mathrm{SMR}}=5 \mu \mathrm{m}$. Results from Eulerian (solid black lines) and Lagrangian (dashed red lines) solutions. Left: Spray volume fraction for droplets without evaporation. Right: Evaporated fuel mass fraction for evaporating droplets with a constant evaporation velocity.

\subsection{Injection of high inertia droplets}

In case of a droplet population with $r_{\mathrm{SMR}}=20 \mu \mathrm{m}$, results from fields of spray volume fraction, spray velocity, gas velocity are illustrated in Fig. 18, whereas Fig. 19 shows the field of vaporized fuel mass fraction. Through spray velocity and spray volume fraction fields, differences between Eulerian and Lagrangian sprays can be observed. There is a high concentration of Eulerian droplets at the front side of the spray. This situation can be argued through the fact that the presureless gas formalism is adopted for the spray modeling where the spray momentum equation is weakly hyperbolic without any diffusion terms. This also implies that all droplets within a mesh cell at a given time step have the same velocity. This argument is enough to explain high concentrations in the spray but should be expanded on since for low inertia droplets the pressureless approach was working perfectly as compared to the Lagrangian model. In fact, a droplet under high inertia has a long response correlation time, leading to a flow regime characterized through a significant Stokes number. Moreover, recall that the drag force between phases is strongly conditioned by the droplet size. Therefore in a cloud of high inertia polydisperse droplets, the particle trajectory crossing (PTC) effects will naturally arise. Yet, the two-way coupled EMSM method will always fail to capture PTC since the presureless gas assumption does not allow to take into account local velocity fluctuations inside the spray such as in [62]. However, despite the lack of PTC modeling, the Eulerian method is proven to be robust and accurate in case of high inertia droplets also since the core of the Eulerian spray is quite similar to the Lagrangian one. Moreover, the high droplet accumulation is drastically reduced in the case of evaporating droplets. The field of evaporated fuel mass fraction between the Lagrangian and the Eulerian sprays, as shown in Fig. 19, matches quite well. As illustrated in Fig. 20, the quantitative evaluation for non evaporating spray along the injection width shows an important gap in spray volume fraction field. However, the Eulerian fuel vapor width tends to the Lagrangian solution, 

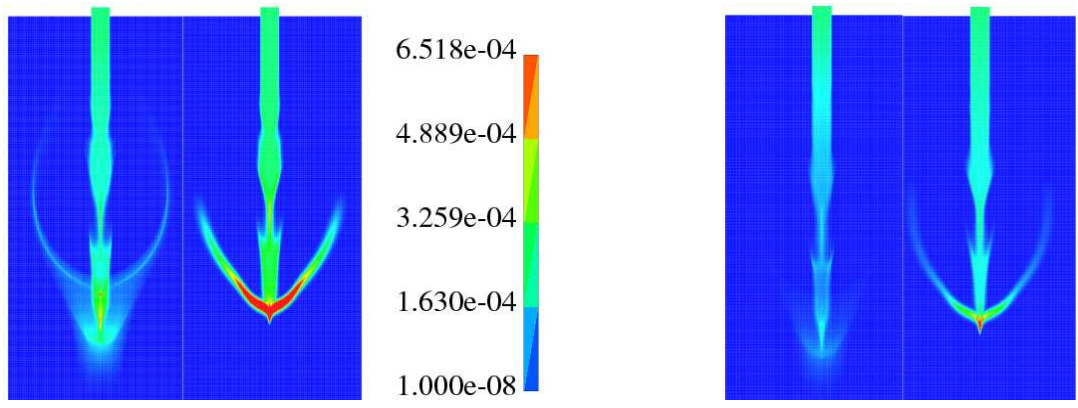

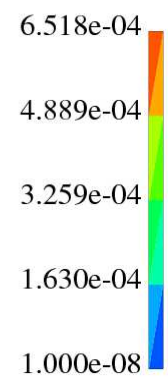
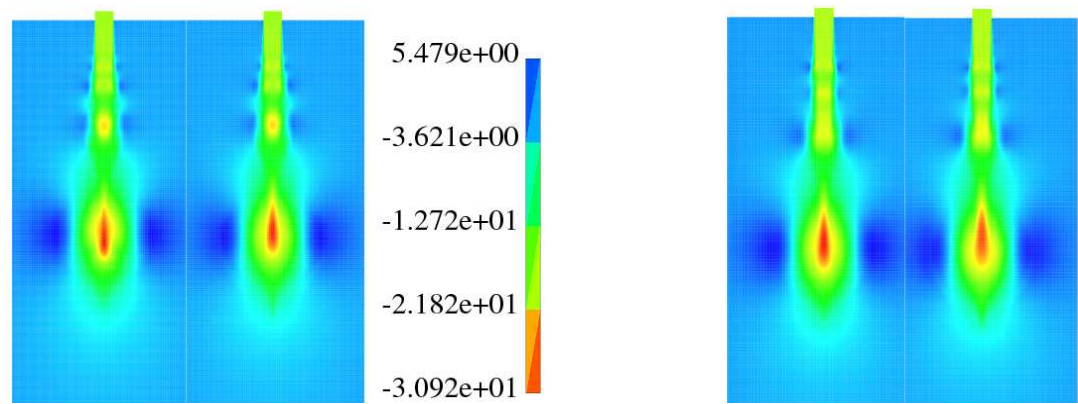

$5.033 e+00$
$-3.641 e+00$
$-1.232 e+01$
$-2.099 e+01$
$-2.966 e+01$
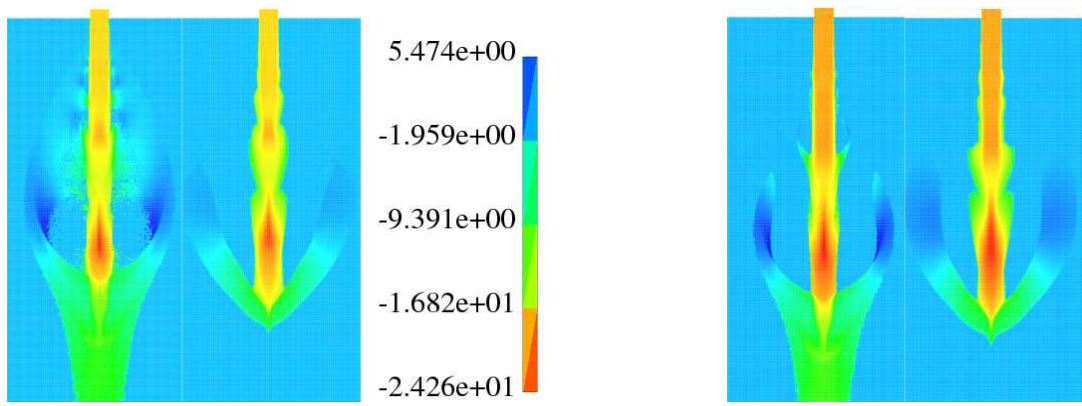

$4.887 e+00$
$-1.904 e+00$
$-8.695 e+00$
$-1.549 e+01$
$-2.228 e+01$

Figure 18: Results for a droplet population of $r_{\mathrm{SMR}}=20 \mu \mathrm{m}$ at time $1.4 \cdot 10^{-2} \mathrm{~s}$. Left: Spray without evaporation. Right: Spray with with constant evaporation velocity. Spray volume fraction (top row), gas velocity field along the $y$-axis (middle row), spray velocity along the $y$-axis (bottom row). In each panel, Lagrangian spray is displayed on the left side whereas Eulerian is displayed on the right side.
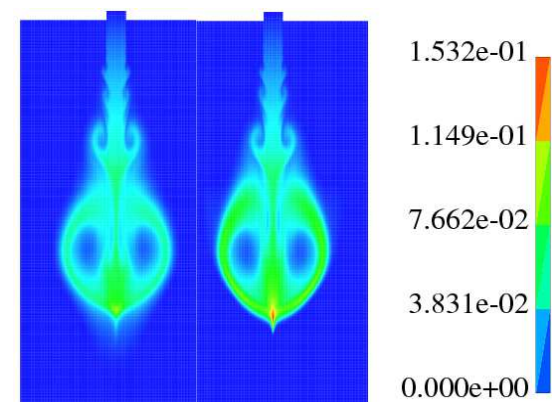

Figure 19: Evaporated fuel mass fraction inside the gas-phase for a droplet population of $r_{\mathrm{SMR}}=20 \mu \mathrm{m}$ under a constant evaporation velocity at time $1.4 \cdot 10^{-2} \mathrm{~s}$. In the panel, Lagrangian spray is displayed on the left side whereas Eulerian is displayed on the right side.

when evaporation is considered. Figure 21 implies that the sudden increase in Eulerian spray volume fraction at the front side of the spray is not observed for the field of evaporated fuel mass fraction. This 
behavior is expected since the evaporation leads to a decreasing droplet inertia where the mono-kinetic (presureless gas) assumption without the polydispersity in velocity, works quite well. This situation is likely to occur, because in normal ICE engine operating conditions, the fuel droplet evaporation takes place under high temperatures and pressures.
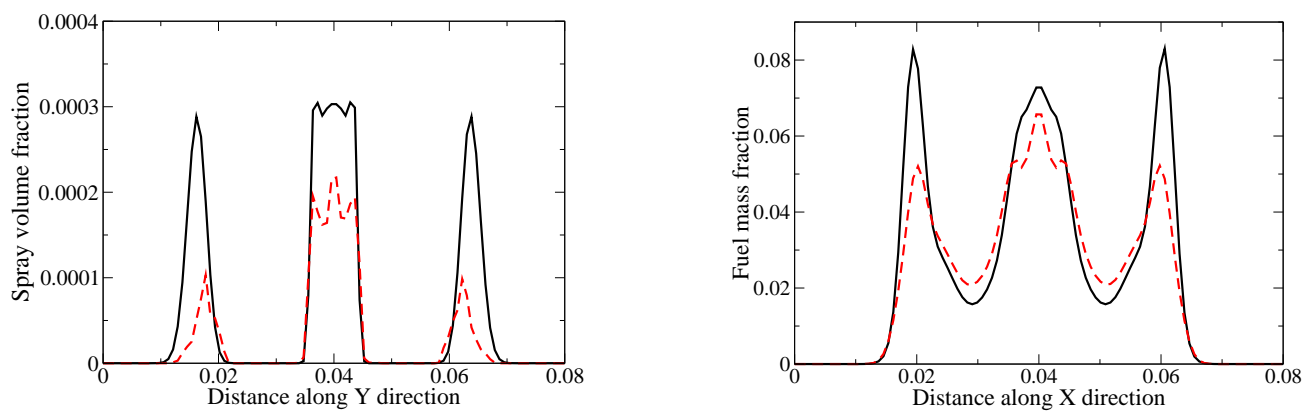

Figure 20: The results from 1-D plots, taken along the $x$-direction which center point on Y axis is 12 diameter far from the injector, in case of a droplet population of $r_{\mathrm{SMR}}=20 \mu \mathrm{m}$. Results from Eulerian (solid black lines) and Lagrangian (dashed red lines) solutions. Left: Spray volume fraction for droplets without evaporation. Right: Evaporated fuel mass fraction for evaporating droplets with a constant evaporation velocity.
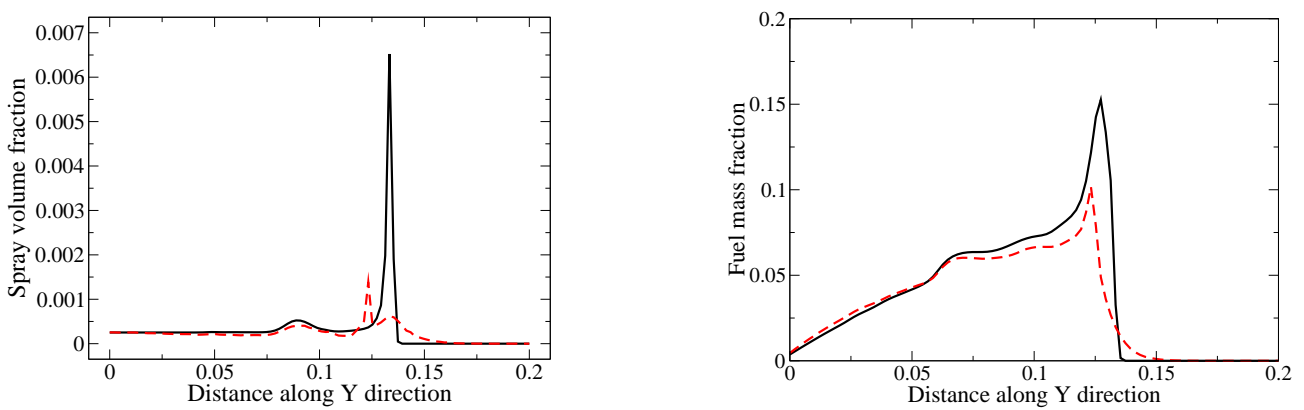

Figure 21: Results from 1-D plots, taken along the $y$-axis see Figure 13, in the case of a droplet population of $r_{\mathrm{SMR}}=20 \mu \mathrm{m}$. Results from Eulerian (solid black lines) and Lagrangian (dashed red lines) solutions. Left: Spray volume fraction for droplets without evaporation. Right: Evaporated fuel mass fraction for evaporating droplets with a constant evaporation velocity.

\subsection{Injection of low inertia droplets under mesh movement}

The configuration of the study conducted in part 6.4 is now extended in order to verify the accuracy and the robustness of the Eulerian two-way coupled polydisperse method with mesh movement. As discussed in part 6.1, the bottom part of the chamber in Fig. 13 is now a moving piston with the crank angle degree (cad) ranging in $\left[-180^{\circ}, 180^{\circ}\right]$. Within the framework of internal combustion engine studies, one takes the length of the piston stroke as $S_{l}=0.08 \mathrm{~m}$ under the rotational speed of the crankshaft which is $N_{\text {rot }}=3000 \mathrm{tr} / \mathrm{min}$. That is, the mean piston speed and its associated characteristic time scale are equal to $U_{m, \text { piston }}=8 \mathrm{~m} / \mathrm{s}$, according to formula given in part 6.1 , and $\tau_{\text {piston }}=0.02 \mathrm{~s}$. Let us point out that $\tau_{\text {piston }}$ and $\tau_{v}$ have the same order of magnitude according to value of $\tau_{v}$ in Table 3 .

Eulerian and Lagrangian sprays volume fractions are displayed in Fig. 22 at cad $=-140^{\circ}-90^{\circ}, 0^{\circ}, 40^{\circ}$. As done in the non moving case, the fuel vapor mass fraction and the spray velocity along the y-axis are displayed in Fig. 23 and Fig. 24, for the same crank angles. One can see that both Eulerian and Lagrangian sprays are in a very good agreement even in case where mesh distorsion and stretching occur during the computations, proving also the robustness of Eulerian simulations as compared to Lagrangian ones. 

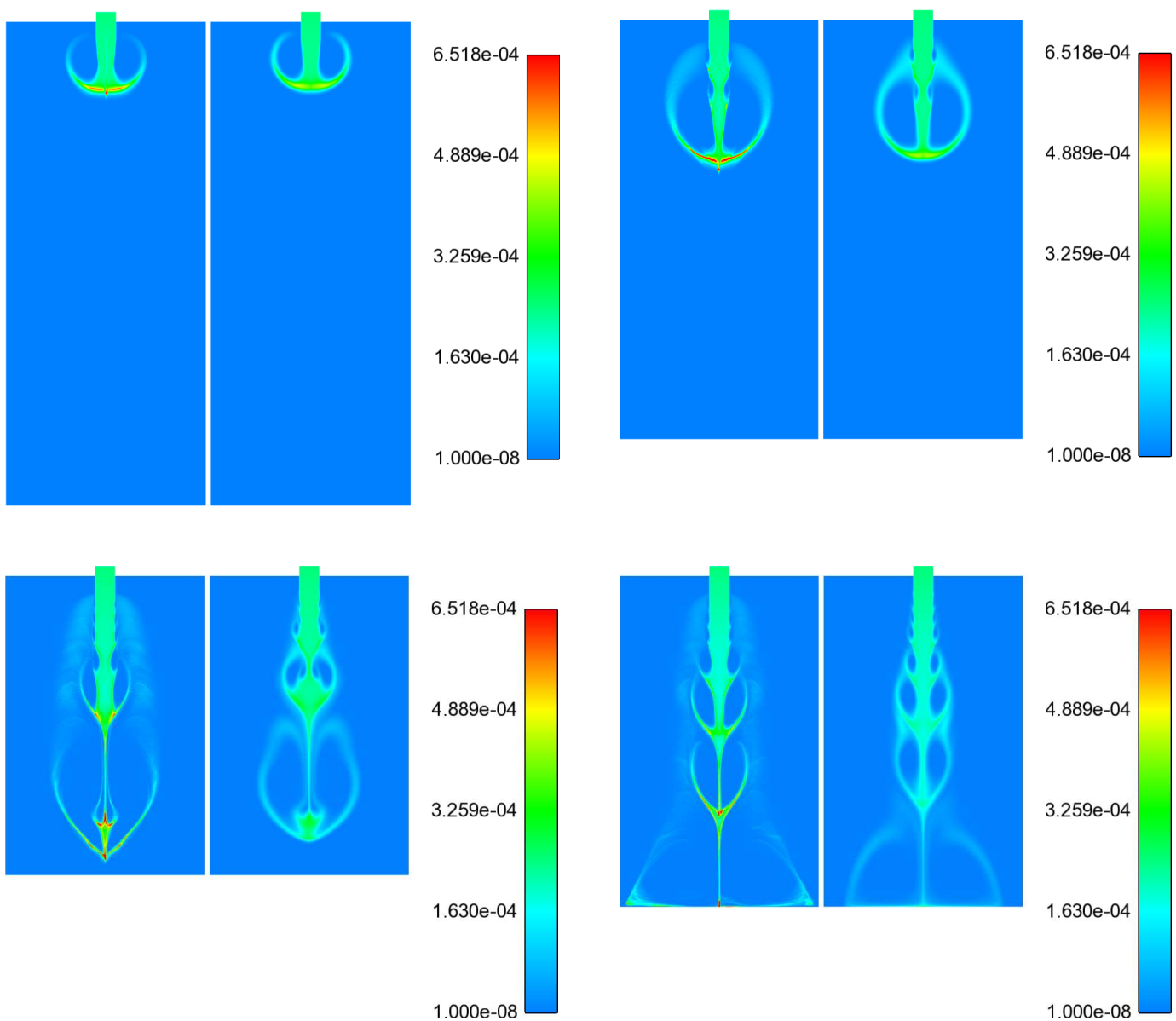

Figure 22: Spray volume fraction results for an evaporating droplet population of $r_{\mathrm{SMR}}=5 \mu \mathrm{m}$ with mesh motion, at crank angle degrees: $-140^{\circ}$ (top left), $-90^{\circ}$ (top right), $0^{\circ}$ (bottom left), $40^{\circ}$ (bottom right). In each panel, Lagrangian spray is displayed on the left side whereas Eulerian is displayed on the right side.

\subsection{Concluding remarks on injection simulations}

In this section, the unexplored issue of the EMSM moment method ability to tackle the two-way polydisperse interactions of droplets with the surrounding gas phase has been revealed. The method has been proved to be stable and accurate, preserving the moment space, in a multi-dimensional context. Through its rigorous comparison with the widely used Lagrangian method, promising results towards ICE engine injection applications have been obtained. The good quality on the fuel vapor field and gas phase properties shows that Eulerian method is in equal footing with the Lagrangian method, being with fixed or moving geometry. The simulations were done under the fully coupled two-way formalism integrated in the ALE algorithm as presented in the previous sections.

As far as the computational cost of the method is concerned, the Eulerian method is twice to three times more expensive than the Lagrangian one in a sequential case but can offer a much better scalability in parallel computations and represent thus the same computational cost in a parallel execution. Nevertheless, one has to note that to conduct a relevant estimation of computational cost between two completely different approaches, one requires a comparison within the same level of information. Lagrangian simulations provide a single realization of the flow, whereas Eulerian relate to a statistical approach. Therefore many Lagrangian simulations would be needed to obtain the Eulerian method level of information. 

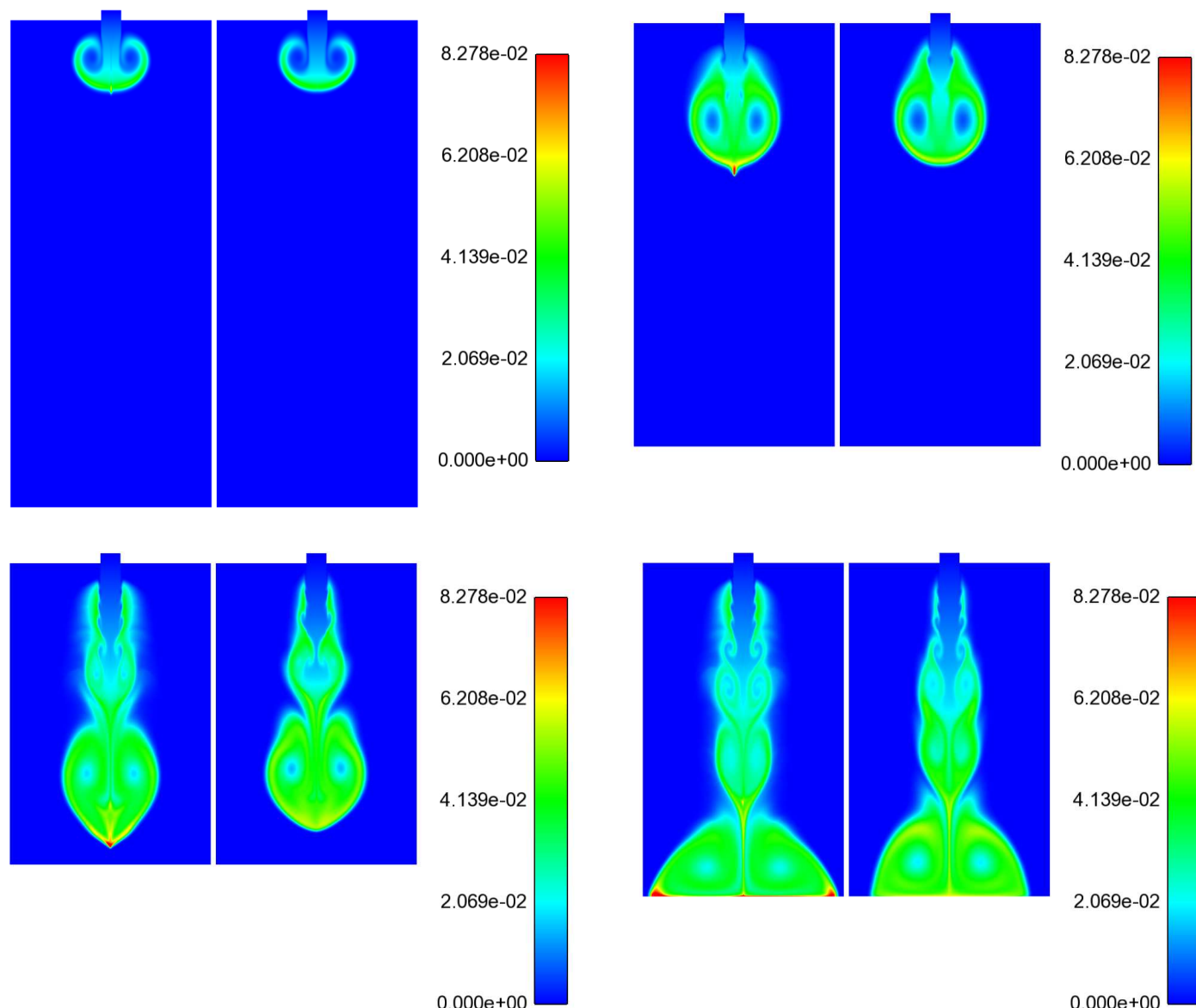

$8.278 \mathrm{e}-02$
$6.208 \mathrm{e}-02$
$4.139 \mathrm{e}-02$
$2.069 \mathrm{e}-02$

$0.000 \mathrm{e}+00$

Figure 23: Fuel vapour mass fraction results for an evaporating droplet population of $r_{\mathrm{SMR}}=5 \mu \mathrm{m}$ with mesh motion, at crank angle degrees: $-140^{\circ}$ (top left), $-90^{\circ}$ (top right), $0^{\circ}$ (bottom left), $40^{\circ}$ (bottom right). In each panel, Lagrangian spray is displayed on the left side whereas Eulerian is displayed on the right side.

\section{Conclusion}

Throughout this paper, we have explained how to adapt the EMSM model and its associated numerical methods from the one-way to the two-way coupling framework and from fixed to moving grids. The challenge here is to respect the strong stability conditions inherent to the model in order to provide robustness without introducing a loss of both computational efficiency and accuracy by enforcing the stability conditions a posteriori. A significant part of this work has consisted in implementing the EMSM model in the IFP-C3D code, an industrial software solving reactive compressible flows in unstructured grids and dedicated to engine computations. By extensive numerical simulations, we have shown that injection cases can be handled.

High order moment methods are able to describe polydispersity without discretizing the size phase space into sections, contrary to sectional/multi-fluid methods. But the methods suggested in the literature $[51,27]$ face some limitations preventing their use in the applications we target. In this respect, the EMSM model can be considered a breakthrough both in terms of modeling, with a new and efficient way to describe polydispersity and in terms of numerical methods, with a low level of artificial dissipation while maintaining the realizability condition and the maximum principle. It currently represents an important source of ongoing investigations, which widens its range of applicability. Resolving one additional moment transport equation, Vié et al. [64] has introduced the ability of describing a correlation between the size 

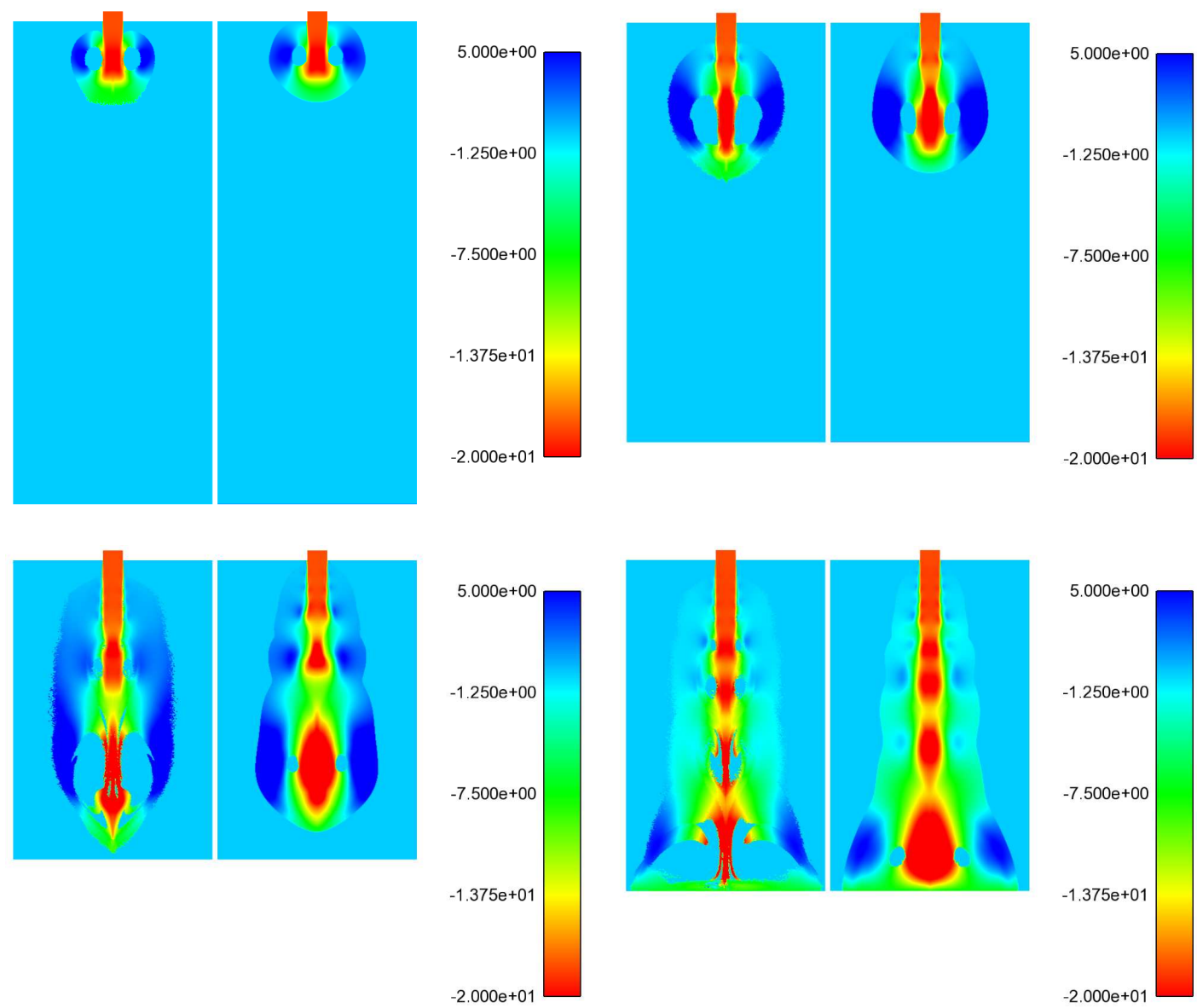

Figure 24: Spray velocity along y-axis results for an evaporating droplet population of $r_{\mathrm{SMR}}=5 \mu \mathrm{m}$ with mesh motion, at crank angle degrees: $-140^{\circ}$ (top left), $-90^{\circ}$ (top right), $0^{\circ}$ (bottom left), $40^{\circ}$ (bottom right). In each panel, Lagrangian spray is displayed on the left side whereas Eulerian is displayed on the right side.

and the velocity variable. Hence, the type of flows described by the EMSM model should describe flows with particles having a larger Stokes number. This extended model has been denoted CSVM model (Coupled Size and Velocity Moment) model. Furthermore, the use of the model and methods developed in this paper in order to treat size polydispersity can be used beyond a mono-kinetic assumption for the particle velocity distribution within the framework of direct numerical simulation. Indeed, the same ideas can be used in order to treat a set of larger Stokes numbers when particle trajectory crossing has to be taken into account, either deterministically such as in [37] using QMOM in velocity phase space, even if it leads to weakly hyperbolic systems of conservation laws and potential singularities [11], or statistically such as in [63, 62], or even treating both types such as in [10]. Besides, it can also be used in the context of turbulence modeling, for example in a RANS framework [22, 23].

\section{Appendix A. Specific aspects of time-space discretization}

We report here some mandatory technicalities at the fully discrete level of the ALE splitting strategy sketched out in §3.3. A significant part of these stems from the use of staggered grids, which is imposed to us by the IFP-C3D environment. 
Appendix A.1. Phase A in the one-way coupling framework

The equations to be solved are

$$
\begin{aligned}
\partial_{t}\left(m_{0}\right) & =-K \tilde{n}(\boldsymbol{m}, 0), \\
\partial_{t}\left(m_{1}\right) & =-K m_{0}, \\
\vdots & \vdots \\
\partial_{t}\left(m_{N}\right) & =-N K m_{N-1}, \\
\partial_{t}\left(m_{1} \boldsymbol{u}_{\boldsymbol{d}}\right) & =\underbrace{-K m_{0} \boldsymbol{u}_{\boldsymbol{d}}}_{\mathrm{A}_{1}}+\underbrace{\frac{18 \pi}{\rho_{\boldsymbol{d}}} \mu_{g} m_{0}\left(\boldsymbol{u}_{\boldsymbol{g}}-\boldsymbol{u}_{\boldsymbol{d}}\right.}_{\mathrm{A}_{0}})
\end{aligned}
$$

for the spray, and

$$
\partial_{t}\left(\rho_{g}\right)=\partial_{t}\left(\rho_{g} \boldsymbol{u}_{\boldsymbol{g}}\right)=\partial_{t}\left(\rho_{g} E_{g}\right)=0
$$

for the gas. The simplicity of this system suggests a further splitting into a drag step $A_{0}$ and an evaporation step $A_{1}$. Since $A_{1}$ can be done using the Massot et al. [48], let us see how to solve $A_{0}$. The $\mathrm{A}_{0}$ equations are

$$
\begin{array}{cl}
\partial_{t}\left(m_{0}\right) & =0 \\
\partial_{t}\left(m_{1}\right) & =0 \\
\vdots & \vdots \\
\partial_{t}\left(m_{N}\right) & =0 \\
\partial_{t}\left(m_{1} \boldsymbol{u}_{\boldsymbol{d}}\right) & =\frac{18 \pi}{\rho_{d}} \mu_{g} m_{0}\left(\boldsymbol{u}_{\boldsymbol{g}}-\boldsymbol{u}_{\boldsymbol{d}}\right),
\end{array}
$$

for the spray, and (A.2) for the gas. From the first $N+1$ equations of (A.3), we have $\boldsymbol{m}(t)=\boldsymbol{m}^{n}$. From (A.2), we have $\mu_{g}(t)=\mu_{g}^{n}$. The spray momentum equation (A.3d) becomes

$$
\partial_{t} \boldsymbol{u}_{\boldsymbol{d}}=\alpha^{n}\left(\boldsymbol{u}_{\boldsymbol{g}}-\boldsymbol{u}_{\boldsymbol{d}}\right), \quad \alpha^{n}=\frac{18 \pi}{\rho_{d}} \frac{\mu_{g}^{n} m_{0}^{n}}{m_{1}^{n}}
$$

the solution of which is

$$
\boldsymbol{u}_{\boldsymbol{d}}(t)=\exp \left(-\alpha^{n}\left(t-t^{n}\right)\right) \boldsymbol{u}_{\boldsymbol{d}}^{n}+\left(1-\exp \left(-\alpha^{n}\left(t-t^{n}\right)\right)\right) \boldsymbol{u}_{\boldsymbol{g}}^{n}
$$

Space discretization over a staggered grid is taken into account in a way similar to $\S 3.1$. Leaving the 3-D extension to readers, we write down the details for a 1-D mesh using the same notations as in $\S 3.1$. By integrating of (A.3d) over the dual cell $\left[x_{i}, x_{i+1}[\right.$ and by invoking approximations in the same vein as (3.11), we end up with

$$
\partial_{t} \boldsymbol{u}_{\boldsymbol{d} i+1 / 2}=\alpha_{i+1 / 2}^{n}\left(\boldsymbol{u}_{\boldsymbol{g}_{i+1 / 2}}-\boldsymbol{u}_{\boldsymbol{d i + 1 / 2}}\right), \quad \alpha_{i+1 / 2}^{n}=\frac{18 \pi}{\rho_{d}} \frac{\Delta x_{i} \mu_{g, i}^{n} m_{0, i}^{n}+\Delta x_{i+1} \mu_{g, i+1}^{n} m_{0, i+1}^{n}}{\Delta x_{i} m_{1, i}^{n}+\Delta x_{i+1} m_{1, i+1}^{n}} .
$$

The solution of this equation is

$$
\boldsymbol{u}_{\boldsymbol{d}_{i+1 / 2}}^{\mathrm{A}_{0}}=\exp \left(-\alpha_{i+1 / 2}^{n} \Delta t\right) \boldsymbol{u}_{\boldsymbol{d}_{i+1 / 2}}^{n}+\left(1-\exp \left(-\alpha_{i+1 / 2}^{n} \Delta t\right)\right) \boldsymbol{u}_{\boldsymbol{g}_{i+1 / 2}}^{n} .
$$

Appendix A.2. Phase B

To alleviate notations without loss of generality, let us examine the one-dimensional case. The 1-D version of system (3.20) reads

$$
\begin{array}{ll}
\partial_{t}\left(J_{d}\right)-J_{d} \partial_{x}\left(u_{d}\right) & =0, \\
\partial_{t}\left(J_{d} \boldsymbol{m}\right) & =0, \\
\partial_{t}\left(J_{d} m_{1} u_{d}\right) & =0 .
\end{array}
$$


The mass balances (A.8b) are discretized on primal cells as

$$
\Delta x_{i}^{B} \boldsymbol{m}_{i}^{B}=\Delta x_{i}^{A} \boldsymbol{m}_{i}^{A} .
$$

The momentum balance (A.8c) is discretized on dual cells as

$$
\frac{1}{2}\left(\Delta x_{i}^{B} m_{1, i}^{B}+\Delta x_{i+1}^{B} m_{1, i+1}^{B}\right) u_{d_{i+1 / 2}^{B}}^{B}=\frac{1}{2}\left(\Delta x_{i}^{A} m_{1, i}^{A}+\Delta x_{i+1}^{A} m_{1, i+1}^{A}\right) u_{d_{i+1 / 2}^{A}}^{A} .
$$

Inserting (A.9) into (A.10) and gives

$$
u_{d_{i+1 / 2}^{B}}^{B}=u_{d_{i+1 / 2}^{A}}^{A},
$$

after simplification. The vertices are displaced by

$$
x_{i+1 / 2}^{B}=x_{i+1 / 2}^{A}+u_{d_{i+1 / 2}}^{B} \Delta t,
$$

from which it follows that

$$
\Delta x_{i}^{B}=\Delta x_{i}^{A}+\left(u_{d_{i+1 / 2}^{B}}^{B}-u_{d_{i-1 / 2}}^{B}\right) \Delta t=\Delta x_{i}^{n}+\left(u_{d_{i+1 / 2}^{A}}^{A}-u_{d_{i-1 / 2}}^{A}\right) \Delta t .
$$

This enables us to retrieve $\boldsymbol{m}_{i}^{B}$ from (A.9). A condition on $\Delta t$ is obviously needed to ensure $\Delta x_{i}^{B}>0$. We impose the sufficient CFL-like condition

$$
\frac{\Delta t}{\Delta x_{i}^{A}} \max \left\{\left|u_{d_{i-1 / 2}}^{A}\right|,\left|u_{d i+1 / 2}^{A}\right|\right\}<\frac{1}{2} .
$$

\section{Appendix A.3. Phase C} form

We apply dimensional splitting to system (3.25), which produces a sequence of 1-D systems of the

$$
\begin{array}{lll}
\partial_{t}\left(J_{d}\right)+J_{d} \partial_{x}\left(u_{d}-w\right) & =0, \\
\partial_{t}\left(J_{d} \boldsymbol{m}\right)+J_{d} \partial_{x}\left(\boldsymbol{m}\left(u_{d}-w\right)\right) & =0, \\
\partial_{t}\left(J_{d} m_{1} \boldsymbol{u}_{\boldsymbol{d}}\right)+J_{d} \partial_{x}\left(m_{1} \boldsymbol{u}_{\boldsymbol{d}}\left(u_{d}-w\right)\right) & =0 .
\end{array}
$$

Consider just one of the above 1-D system. Then, the vertices are displaced by

$$
x_{i+1 / 2}^{n+1}=x_{i+1 / 2}^{B}-\left(u_{d_{i+1 / 2}^{B}}^{B}-w_{i+1 / 2}^{n}\right) \Delta t=x_{i+1 / 2}^{n}+w_{i+1 / 2}^{n} \Delta t,
$$

from which it follows that

$$
\begin{aligned}
\Delta x_{i}^{n+1} & =\Delta x_{i}^{B}-\left(u_{d i+1 / 2}^{B}-u_{d_{i-1 / 2}^{B}}^{B}\right) \Delta t+\left(w_{i+1 / 2}^{n}-w_{i-1 / 2}^{n}\right) \Delta t \\
& =\Delta x_{i}^{n}+\left(w_{i+1 / 2}^{n}-w_{i-1 / 2}^{n}\right) \Delta t .
\end{aligned}
$$

The mass balances (A.14b) are discretized on primal cells by the explicit scheme

$$
\Delta x_{i}^{n+1} \boldsymbol{m}_{i}^{n+1}=\Delta x_{i}^{B} \boldsymbol{m}_{i}^{B}-\Delta t\left(\boldsymbol{Q}_{i+1 / 2}^{B}-\boldsymbol{Q}_{i-1 / 2}^{B}\right),
$$

for some numerical fluxes $\boldsymbol{Q}^{B}$, required to be homogeneous to $\boldsymbol{m}\left(u_{d}-w\right)$. Inspired by the approach of Kah et al. [39] for the slightly different system $\partial_{t}(\boldsymbol{m})+\partial_{x}\left(\boldsymbol{m} u_{d}\right)=0$, we consider

$$
\boldsymbol{Q}_{i+1 / 2}^{B}=\left\{\begin{array}{lll}
\frac{1}{\Delta t} \int_{x_{i+1 / 2}^{B}-\left(u_{d_{i+1 / 2}^{B}}^{B}-w_{i+1 / 2}^{n}\right) \Delta t}^{x_{i+1 / 2}^{B}} & \hat{\boldsymbol{m}}_{i}^{B}(x) \mathrm{d} x & \text { if } u_{d_{i+1 / 2}^{B}}^{B}-w_{i+1 / 2}^{n}>0, \\
\frac{1}{\Delta t} \int_{x_{i+1 / 2}^{B}}^{x_{i+1 / 2}^{B}-\left(u_{d_{i+1 / 2}}^{B}-w_{i+1 / 2}^{n}\right) \Delta t} \hat{\boldsymbol{m}}_{i+1}^{B}(x) \mathrm{d} x & \text { if } u_{d_{i+1 / 2}^{B}}^{B}-w_{i+1 / 2}^{n}<0,
\end{array}\right.
$$

for some reconstructions $\hat{\boldsymbol{m}}_{i}^{B}$ (.) of $\hat{\boldsymbol{m}}^{B}$ over the cells $i \in \mathbb{Z}$. Formula (A.17) is clearly consistent with the basic first-order upwind flux

$$
\boldsymbol{Q}_{i+1 / 2}^{B}=\boldsymbol{m}_{i}^{B}\left(u_{d}^{B}-w^{n}\right)_{i+1 / 2}^{+}+\boldsymbol{m}_{i+1}^{B}\left(u_{d}^{B}-w^{n}\right)_{i+1 / 2}^{-},
$$


when $\hat{\boldsymbol{m}}_{i}^{B}(x)=\boldsymbol{m}_{i}^{B}$ is constant over cell $i$. To enhance accuracy for the transport of moments, let $\boldsymbol{p}=\left(p_{1}, \ldots, p_{N}\right)$ be the canonical moments introduced in $\S 2.4$. We set

$$
\hat{\boldsymbol{m}}_{i}^{B}(x)=\boldsymbol{M}\left(\hat{m}_{0, i}^{B}(x) ; \hat{\boldsymbol{p}}_{i}^{B}(x)\right),
$$

where the function

$$
\boldsymbol{M}\left(m_{0} ; \boldsymbol{p}\right)=m_{0}\left(\begin{array}{c}
1 \\
p_{1} \\
p_{1}\left(\left(1-p_{1}\right) p_{2}+p_{1}\right) \\
p_{1}\left(\left(1-p_{1}\right) p_{2}\left(1-p_{2}\right) p_{3}+\left(p_{1}+p_{2}\left(1-p_{1}\right)\right)^{2}\right) \\
\cdots
\end{array}\right)
$$

returns $\boldsymbol{m}=\left(m_{0}, m_{1}, \ldots, m_{N}\right)$ as a function of $\left(m_{0} ; p_{1}, \ldots, p_{N}\right)$, and

$$
\begin{array}{rc}
\hat{m}_{0, i}^{B}(x)=m_{0, i}^{B}+D m_{0, i}\left(x-x_{i}^{B}\right), \\
\hat{p}_{1, i}^{B}(x)=\bar{p}_{1, i}^{B}+D p_{1, i}\left(x-x_{i}^{B}\right), \\
\vdots & \vdots \\
\hat{p}_{N, i}^{B}(x)=\bar{p}_{N, i}^{B}+D p_{N, i}\left(x-x_{i}^{B}\right),
\end{array}
$$

are the piecewise affine reconstructions. These reconstructions involve some limited slopes $D p_{k, i}$ and some modified averages $\bar{p}_{k, i}$, which are linearly related to the slopes by

$$
\bar{p}_{k, i}=a_{k, i}+b_{k, i} D p_{k, i}
$$

in order to preserve the mean values of the moments $\left(m_{1}, \ldots, m_{N}\right)$. The calculation of the coefficients $a_{2 j}, a_{3 j}$ and $b_{2 j}, b_{3 j}$, presented in [39], is completed using Maple ${ }^{10}$, and implemented in the Fortran code. Notwithstanding some heaviness, their algebraic expressions do not entail sensible extra CPU cost.

The slopes for the canonical moments are determined using limiters in order to comply with the maximum principle

$$
r_{k, i} \leq p_{k}(x) \leq R_{k, i}, \quad \text { for all } x \in\left[x_{i-1 / 2}, x_{i+1 / 2}[,\right.
$$

where $r_{k, i}=\min \left\{p_{k, i-1}^{B}, p_{k, i}^{B}, p_{k, i+1}^{B}\right\}$ and $R_{k, i}=\max \left\{p_{k, i-1}^{B}, p_{k, i}^{B}, p_{k, i+1}^{B}\right\}$. By linearity, condition (A.23) is equivalent to

$$
r_{k, i} \leq a_{k, i}+b_{k, i} D p_{k, i} \pm \frac{1}{2} \Delta x_{i}^{B} D p_{k, i} \leq R_{k, i} .
$$

Solving for the slope, we get

$$
\min \left\{\frac{r_{k, i}-a_{k, i}}{b_{k, i}+\frac{1}{2} \Delta x_{i}^{B}}, \frac{a_{k, i}-R_{k, i}}{\frac{1}{2} \Delta x_{i}^{B}-b_{k, i}}\right\} \leq D p_{k, i} \leq \min \left\{\frac{R_{k, i}-a_{k, i}}{b_{k, i}+\frac{1}{2} \Delta x_{i}^{B}}, \frac{a_{k, i}-r_{k, i}}{\frac{1}{2} \Delta x_{i}^{B}-b_{k, i}}\right\} .
$$

In practice, we opt for the limiter

$$
D p_{k, i}=\frac{1}{2}\left(\operatorname{sgn}\left(p_{k, i+1}^{B}-p_{k, i}^{B}\right)+\operatorname{sgn}\left(p_{k, i}^{B}-p_{k, i-1}^{B}\right)\right) \min \left\{\frac{\left|p_{k, i+1}^{B}-a_{k, i}\right|}{\Delta x_{i}^{B}+2 b_{k, i}}, \frac{\left|a_{k, i}-p_{k, i+1}^{B}\right|}{\Delta x_{i}^{B}+2 b_{k, i}}\right\},
$$

which can be checked to satisfy (A.25). The same limiter is used for the slope on the droplet number $m_{0}$. In order to ensure the non-negativity of $m_{0}$, the additional condition $\Delta x_{i}^{B} D m_{0, i} \leq 2 m_{0, i}^{B}$ is require. This ensures that the extremum of $\hat{m}_{0, i}(\cdot)$, which occurs at one of the cell bound, remains positive. Finally, it can be shown that a sufficient stability condition for this moment transport scheme is

$$
\frac{\Delta t}{\Delta x_{i}^{B}} \max \left\{\left|u_{d_{i-1 / 2}^{B}}^{B}-w_{i-1 / 2}^{n}\right|,\left|u_{d i+1 / 2}^{B}-w_{i+1 / 2}^{n}\right|\right\}<\frac{1}{2} .
$$

\footnotetext{
${ }^{10}$ Maplesoft, a division of Waterloo Maple, 2007
} 
The momentum balance (A.14c) is discretized over dual cells by the explicit scheme

$$
\frac{1}{2}\left(\Delta x_{i}^{n+1} m_{1, i}^{n+1}+\Delta x_{i+1}^{n+1} m_{1, i+1}^{n+1}\right) \boldsymbol{u}_{\boldsymbol{d}_{i+1 / 2}^{n+1}}^{n+1}=\frac{1}{2}\left(\Delta x_{i}^{B} m_{1, i}^{B}+\Delta x_{i+1}^{B} m_{1, i+1}^{B}\right) \boldsymbol{u}_{\boldsymbol{d}_{i+1 / 2}^{B}}^{B}-\Delta t\left(\boldsymbol{\Xi}_{i+1}^{B}-\boldsymbol{\Xi}_{i}^{B}\right),
$$

using the numerical flux

$$
\boldsymbol{\Xi}_{i}^{B}=\boldsymbol{u}_{\boldsymbol{d}_{i-1 / 2}^{B}}\left(\frac{Q_{1, i-1 / 2}^{B}+Q_{1, i+1 / 2}^{B}}{2}\right)^{+}+\boldsymbol{u}_{\boldsymbol{d}_{i+1 / 2}^{B}}\left(\frac{Q_{1, i-1 / 2}^{B}+Q_{1, i+1 / 2}^{B}}{2}\right)^{-}
$$

where $Q_{1, i+1 / 2}^{B}$ is the $m_{1}$-component of the flux $\boldsymbol{Q}_{i+1 / 2}^{B}$ defined in (A.17). This allows the maximum principle

$$
\min \left\{\boldsymbol{u}_{\boldsymbol{d}_{i-1 / 2}^{B}}^{B}, \boldsymbol{u}_{\boldsymbol{d}_{i+1 / 2}^{B}}^{B}, \boldsymbol{u}_{\boldsymbol{d} i+3 / 2}\right\} \leq \boldsymbol{u}_{\boldsymbol{d}_{i+1 / 2}^{n+1}}^{n+\max }\left\{\boldsymbol{u}_{\boldsymbol{d}_{i-1 / 2}}^{B}, \boldsymbol{u}_{\boldsymbol{d}_{i+1 / 2}^{B}}^{B}, \boldsymbol{u}_{\boldsymbol{d}_{i+3 / 2}^{B}}^{B}\right\},
$$

to hold componentwise on $\boldsymbol{u}_{\boldsymbol{d}}$, provided some lengthy stability criterion is met. This criterion is obtained writing that the velocity update in (A.28) is a convex combination of the velocities at face $B$. This criterion gives us the maximum principle on the velocity. This scheme is first-order with respect to $\boldsymbol{u}_{\boldsymbol{d}}$, but we content ourselves with this accuracy for the velocity.

\section{References}

[1] Allaire, G., Clerc, S., Kokh, S., 2002. A five-equation model for the simulation of interfaces between compressible fluids. J. Comput. Phys. 181, 577-616.

[2] Amsden, A., O'Rourke, P.J., Butler, T.D., 1989. KIVA II: A computer program for chemically reactive flows with sprays. Technical Report LA-11560-MS. Los Alamos National Laboratory. Los Alamos, New Mexico.

[3] Baer, M.R., Nunziato, J.W., 1986. A two-phase mixture theory for the deflagration-to-detonation transition (DDT) in reactive granular materials. Int. J. Multiphase Flow 12, 861-889.

[4] Balachandar, S., Eaton, J.K., 2010. Turbulent dispersed multiphase flow. Ann. Rev. Fluid Mech. 42, 111-133.

[5] Bohbot, J., Gillet, N., Benkenida, A., 2009. IFP-C3D: an unstructured parallel solver for reactive compressible gas flow with spray. Oil Gas Sci. Tech. 64, 309-335.

[6] Bouchut, F., 1994. On zero pressure gas dynamics, in: Perthame, B. (Ed.), Advances in kinetic theory and computing. World Science Publisher, River Edge, NJ. volume 22 of Series on Advances in Mathematics for Applied Sciences, pp. 171-190.

[7] Bouchut, F., Jin, S., Li, X., 2003. Numerical approximations of pressureless and isothermal gas dynamics. SIAM J. Numer. Anal. 41, 135-158.

[8] Capecelatro, J., Desjardins, O., 2013. An Euler-Lagrange strategy for simulating particle-laden flows. J. Comput. Phys. 238, 1-31.

[9] de Chaisemartin, S., Fréret, L., Kah, D., Laurent, F., Fox, R., Reveillon, J., Massot, M., 2009. Eulerian models for turbulent spray combustion with polydispersity and droplet crossing. C. R. Mécanique 337, 438-448. Special Issue Combustion for Aerospace Propulsion.

[10] Chalons, C., Fox, R.O., Massot, M., 2010. A multi-Gaussian quadrature method of moments for gas-particle flows in a LES framework, in: Proceedings of the Summer Program 2010, Center for Turbulence Research, Stanford University, Stanford. pp. 347-358.

[11] Chalons, C., Kah, D., Massot, M., 2012. Beyond pressureless gas dynamics: quadrature-based velocity moment models. Commun. Math. Sci. 10, 1241-1272.

[12] Chenadec, V.L., Pitsch, H., 2013a. A 3d unsplit forward/backward volume-of-fluid approach and coupling to the level set method. J. Comput. Phys. 233, $10-33$.

[13] Chenadec, V.L., Pitsch, H., 2013b. A monotonicity preserving conservative sharp interface flow solver for high density ratio two-phase flows. J. Comput. Phys. 249, $185-203$. 
[14] Coquel, F., Nguyen, Q.L., Postel, M., Tran, Q.H., 2010. Entropy-satisfying relaxation method with large time-steps for Euler IBVPs. Math. Comp. 79, 1493-1533. doi:10.1090/S0025-5718-10-02339-2.

[15] Després, B., Mazeran, C., 2005. Lagrangian gas dynamics in two-dimensions and Lagrangian systems. Arch. Ration. Mech. Anal. 178, 327-372.

[16] Dette, H., Studden, W.J., 1997. The theory of canonical moments with applications in statistics, probability, and analysis. Wiley Series in Probability and Statistics: Applied Probability and Statistics, John Wiley \& Sons Inc., New York. A Wiley-Interscience Publication.

[17] Doisneau, F., Laurent, F., Murrone, A., Dupays, J., Massot, M., 2013. Eulerian multi-fluid models for the simulation of dynamics and coalescence of particles in solid propellant combustion. J. Comput. Phys. 234, 230-262.

[18] Doisneau, F., Sibra, A., Dupays, J., Murrone, A., Laurent, F., Massot, M., 2014. An efficient and accurate numerical strategy for two-way coupling in unsteady polydisperse moderately dense sprays: application to Solid Rocket Motor instabilities. J. Propul. Power 30, 727-748.

[19] Donea, J., Huerta, A., Ponthot, J.P., Rodríguez-Ferran, A., 2004. Arbitrary Lagragian-Eulerian methods, in: Stein, E., de Borst, R., Hughes, T. (Eds.), Encyclopedia of Computational Mechanics. John Wiley \& Sons. volume 1. chapter 14, pp. 413-437.

[20] Duarte, M., Bonaventura, Z., Massot, M., Bourdon, A., Descombes, S., Dumont, T., 2012. A new numerical strategy with space-time adaptivity and error control for multi-scale streamer discharge simulations. J. Comput. Phys. 231, 1002-1019.

[21] Dukowicz, J.K., 1980. A particle-fluid numerical model for liquid sprays. J. Comput. Phys. 35, $229-253$.

[22] Emre, O., 2014. Eulerian modeling and simulation of polydisperse moderately dense sprays in the framework of separated two-phase flows. Ph.D. thesis. École Centrale Paris.

[23] Emre, O., Fox, R., Massot, M., de Chaisemartin, S., Jay, S., Laurent, F., 2014. Eulerian modeling of a polydisperse evaporating spray under realistic internal-combustion-engine conditions. Flow Turbul. Combust., in revision, $1-36$.

[24] Farhat, C., Guezaine, P., Grandmont, C., 2001. The discrete geometric conservation law and the nonlinear stability of ALE schemes for the solution of flow problems on moving grids. J. Comput. Phys. 174, 669-694.

[25] Ferrand, V., Bazile, R., Borée, J., Charnay, G., 2003. Gas droplet turbulent velocity correlations and twophase interaction in an axisymmetric jet laden with partly responsive droplets. Int. J. Multiphase Flow 29, $195-217$.

[26] Fox, R.O., 2007. Fundamental of polydisperse multiphase flows, in: Marchisio, D.L., Fox, R.O. (Eds.), Computational Models for Turbulent Multiphase Reacting Flows. Springer, Vienna. volume 492 of CISM Courses and Lectures, pp. 79-123.

[27] Fox, R.O., Laurent, F., Massot, M., 2008. Numerical simulation of spray coalescence in an Eulerian framework: direct quadrature method of moments and multi-fluid method. J. Comput. Phys. 227, 3058-3088.

[28] Fréret, L., Lacour, C., de Chaisemartin, S., Ducruix, S., Durox, D., Laurent, F., Massot, M., 2009. Pulsated free jets with polydisperse spray injection : Experiments and numerical simulations, in: Proceedings of the Combustion Institute, pp. 2215-2222.

[29] Fréret, L., Thomine, O., Reveillon, J., de Chaisemartin, S., Laurent, F., Massot, M., 2011. On the role of preferential segregation in flame dynamics in polydisperse evaporating sprays, in: Proceedings of the Summer Program 2010, Center for Turbulence Research, Stanford University, pp. 383-392.

[30] Godsave, G.A.E., 1953. Studies of the combustion of drops in a fuel spray: the burning of single drops of fuel, in: Proceedings of the 4th International Symposium on Combustion, The Combustion Institute, Baltimore. pp. $818-830$.

[31] Greenberg, J., Silverman, I., Tambour, Y., 1993. On the origin of spray sectional conservation equations. Combustion and Flame 93, 90-96. 
[32] Habchi, C., 2006. Modélisation tridimensionnelle de l'injection et de la préparation du mélange gaz/carburant dans les moteurs à combustion interne. Mémoire de HDR.

[33] Hairer, E., Wanner, G., 1996. Solving Ordinary Differential Equations. II: Stiff and Differential-Algebraic Problems. volume 2 of Springer Series In Computational Mathematics. Springer, Berlin.

[34] Hirt, C.W., Amsden, A.A., Cook, J.L., 1974. An arbitrary Lagrangian-Eulerian computing method for all flow speeds. J. Comput. Phys. 14, 227-253. doi:DOI : 10.1016/0021-9991(74)90051-5.

[35] Huerta, A., Liu, W., 1988. Viscous flow structure interaction. J. Pressure Vessel Technol. 110, 15-21.

[36] Ishii, M., 1975. Thermo-Fluid dynamic theory of two-phase flow. Eyrolles.

[37] Kah, D., 2010. Taking into account polydispersity for the modeling of liquid fuel injection in internal combustion engines. Ph.D. thesis. École Centrale Paris. http://tel.archives-ouvertes.fr/tel-00618786.

[38] Kah, D., Laurent, F., Fréret, L., de Chaisemartin, S., Fox, R., Reveillon, J., Massot, M., 2010. Eulerian quadrature-based moment models for polydisperse evaporating sprays. Flow Turbul. Combust. 85, 649-676. Special Issue Dedicated to Stephen B. Pope.

[39] Kah, D., Laurent, F., Massot, M., Jay, S., 2012. A high order moment method simulating evaporation and advection of a polydisperse liquid spray. J. Comput. Phys. 231, 394-422.

[40] Kokh, S., Lagoutière, F., 2010. An anti-diffusive numerical scheme for the simulation of interfaces between compressible fluids by means of a five-equation model. J. Comput. Phys. 229, 2773-2809. doi:DOI:10.1016/ j.jcp.2009.12.003.

[41] Laurent, F., Massot, M., 2001. Multi-fluid modeling of laminar polydispersed spray flames: origin, assumptions and comparison of the sectional and sampling methods. Combust. Theor. Model. 5, 537-572.

[42] Le Martelot, S., 2013. Contribution à la simulation numérique directe de l'ébullition. Ph.D. thesis. Université d'Aix Marseille.

[43] LeVeque, R.J., 2002. Finite volume methods for hyperbolic problems. Cambridge Texts in Applied Mathematics, Cambridge University Press, Cambridge.

[44] Mandumpala Devassy, B., Habchi, C., Daniel, E., 2013. A new atomization model for high speed liquid jets using a turbulent, compressible, two-phase flow model and a surface density approach, in: Proceedings of the 25th ILASS, European Conference on Liquid Atomization and Spray Systems, Chania, Greece, pp. 1-8.

[45] Marchisio, D.L., Fox, R.O., 2005. Solution of population balance equations using the direct quadrature method of moments. J. Aerosol Sci. 36, 43-73.

[46] Masi, E., 2010. Theoretical and numerical study of the modeling of unsteady nonisothermal particle-laden turbulent flows by an Eulerian-Eulerian approach. Ph.D. thesis. Institut National Polytechnique de Toulouse.

[47] Massot, M., 2007. Eulerian multi-fluid models for polydisperse evaporating sprays, in: Marchisio, D.L., Fox, R.O. (Eds.), Computational Models for Turbulent Multiphase Reacting Flows. Springer, Vienna. volume 492 of CISM Courses and Lectures, pp. 79-123.

[48] Massot, M., Laurent, F., Kah, D., de Chaisemartin, S., 2010. A robust moment method for evaluation of the disappearance rate of evaporating sprays. SIAM J. Appl. Math. 70, 3203-3234.

[49] McGraw, R., 2007. Numerical advection of correlated tracers: preserving particle size/composition moment sequences during transport of aerosol mixtures. J. Phys.: Conf. Ser. 78, 012045.

[50] Mead, L.R., Papanicolaou, N., 1984. Maximum entropy in the problem of moments. J. Math. Phys. 25, $2404-2417$

[51] Mossa, J.B., 2005. Extension polydisperse pour la description euler-euler des écoulements diphasiques réactifs - TH/CFD/05/74. Ph.D. thesis. Institut National Polytechnique de Toulouse.

[52] O'Rourke, P.J., 1981. Collective drop effects on vaporizing liquid sprays. Ph.D. thesis. Princeton University. 
[53] Patankar, S.V., 1980. Numerical Heat Transfer and Fluid Flow. Hemisphere Publishing Corporation, Washington D. C.

[54] Perthame, B., 2002. Kinetic formulation of conservation laws. volume 21 of Oxford Lecture Series in Mathematics and its Applications. Oxford University Press, Oxford.

[55] Reveillon, J., 2007. DNS of spray combustion, dispersion evaporation and combustion, in: Marchisio, D.L., Fox, R.O. (Eds.), Computational Models for Turbulent Multiphase Reacting Flows. Springer, Vienna. volume 492 of CISM Courses and Lectures, p. 229.

[56] Rosin, P., Rammler, E., 1933. Laws governing the fineness of powdered coal. J. Inst. Fuel , 29-36.

[57] Saurel, R., Metayer, O.L., 2000. A multiphase model for compressible flows with interfaces, shocks, detonation waves and cavitation. J. Fluid Mech. 431, 239-271.

[58] Schiller, L., Nauman, A., 1935. A drag coefficient correlation. VDI Zeitung 77, 318-320.

[59] Spalding, D.B., 1953. The combustion of liquid fuels, in: Proceedings of the 4th International Symposium on Combustion, The Combustion Institute, Baltimore. pp. 847-864.

[60] Torres, D.J., Trujillo, M.F., 2006. KIVA-IV: An unstructured ALE code for compressible gas flow with sprays. J. Comput. Phys. 219, 943-975. doi:10.1016/j.jcp.2006.07.006.

[61] Truchot, B., 2005. Développement et validation d'un modèle eulérien en vue de la simulation des jets de carburants dans les moteurs à combustion interne. Ph.D. thesis. Institut National Polytechnique de Toulouse.

[62] Vié, A., Doisneau, F., Massot, M., 2014. On the anisotropic Gaussian closure for the prediction of inertial-particle laden flows. Commun. Comput. Phys., in press , 1-48Available on HAL, http://hal. archives-ouvertes.fr/hal-00912319.

[63] Vié, A., Jay, S., Cuenot, B., Massot, M., 2013a. Accounting for polydispersion in the eulerian large eddy simulation of the two-phase flow in an aeronautical-type burner. Flow, Turbulence and Combustion 90, $545-581$.

[64] Vié, A., Laurent, F., Massot, M., 2013b. Size-velocity correlations in high order moment methods for polydisperse evaporating sprays: modeling and numerical issues. J. Comput. Phys. 237, 277-310.

[65] Vié, A., Masi, E., Simonin, O., Massot, M., 2012. On the direct numerical simulation of moderate-Stokesnumber turbulent particulate flows using Algebraic-Closure-Based and Kinetic-Based Moment Methods, in: Proceedings of the Summer Program 2012, Center for Turbulence Research, Stanford University, pp. 355-364.

[66] Vikas, V., Wang, Z.J., Passalacqua, A., Fox, R.O., 2011. Realizable high-order finite-volume schemes for quadrature-based moment methods. J. Comput. Phys. 230, 5328-5352. doi:DOI:10.1016/j.jcp.2011.03. 038.

[67] Williams, F.A., 1958. Spray combustion and atomization. Phys. Fluids 1, 541-545.

[68] Wright, D.L., 2007. Numerical advection of moments of the particle size distribution in Eulerian models. J. Aerosol Sci. 38, 352-369. doi:DOI:10.1016/j.jaerosci.2006.11.011.

[69] Yuan, C., Fox, R.O., 2011. Conditional quadrature method of moments for kinetic equations. J. Comput. Phys. in press, corrected proof, 8216-8246. doi:DOI:10.1016/j.jcp.2011.07.020.

[70] Yuan, C., Laurent, F., Fox, R.O., 2012. An extended quadrature method of moments for population balance equations. J. Aerosol Sci. 51, 1-23.

[71] Zolver, M., Klahr, D., Bohbot, J., Laget, O., Torres, A., 2003. Reactive CFD in engines with a new unstructured parallel solver. Oil \& Gas Science and Technology 58, 33-46. 